\title{
MISHEARD ME ORONYMINATOR: USING ORONYMS TO VALIDATE THE CORRECTNESS OF FREQUENCY DICTIONARIES
}

\author{
A Thesis \\ Presented to \\ the Faculty of California Polytechnic State University \\ San Luis Obispo
}

\author{
In Partial Fulfillment \\ of the Requirements for the Degree \\ Master of Science in Computer Science
}

by

Jennifer "Jenee" Gayle Hughes

April 2013 
(c) 2013

Jennifer "Jenee" Gayle Hughes

ALL RIGHTS RESERVED 


\section{COMMITTEE MEMBERSHIP}

TITLE:

Misheard Me Oronyminator: Using Oronyms to Validate the Correctness of Frequency Dictionaries

AUTHOR: Jennifer "Jenee" Gayle Hughes

DATE SUBMITTED: April 2013

COMMITTEE CHAIR: Z Zoë Wood, Ph.D.

COMMITTEE MEMBER: Franz Kurfess, Ph.D.

COMMITTEE MEMBER: John Clements, Ph.D. 


\begin{abstract}
Misheard Me Oronyminator: Using Oronyms to Validate the Correctness of Frequency Dictionaries

Jennifer "Jenee" Gayle Hughes
\end{abstract}

In the field of speech recognition, an algorithm must learn to tell the difference between "a nice rock" and "a gneiss rock". These identical-sounding phrases are called oronyms. Word frequency dictionaries are often used by speech recognition systems to help resolve phonetic sequences with more than one possible orthographic phrase interpretation, by looking up which oronym of the root phonetic sequence contains the most-common words.

Our paper demonstrates a technique used to validate word frequency dictionary values. We chose to use frequency values from the UNISYN dictionary, which tallies each word on a per-occurance basis, using a proprietary text corpus, to calculate word frequency.

In the first phase of our user study, we generated oronym strings for the phrase "a nice cold hour", and had over a dozen people make 62 recordings of the most-common oronyms for that phrase. In the second phase, we selected 15 of the phase one recordings, and had approximately 74 different people transcribe each one, for a total of 953 transcriptions overall.

If the frequency dictionary values for our test phrases accurately reflected the real-world expectations of actual listeners, we would expect that the mostcommonly transcribed phrases in our user study would roughly correspond with our metric for the most likely oronym interpretation of the root phrase.

During the course of our study, we found that using per-occurance frequency 
values, like those found in the UNISYN dictionary, when computing our overallphrase-frequency metric caused the end result to be thrown off by excessively common words, such as "the", "is", and "a". These super-common words had such high per-occurance tallies that they overpowered any effect that any regular word had on a frequency metric. When we used frequency values from the COCA dictionary, which has word frequency values tallied on a document-count basis instead of a UNISYN-like per-occurance basis, we found that this effect was mitigated. As a result, we do not recommend using the UNISYN dictionary for word frequency purposes. 


\section{Contents}

List of Tables $\quad$ ix

List of Figures $\quad$ X

1 Preliminary Vocabulary 1

1.1 Mondegreens . . . . . . . . . . . . . . . . . . . . . . 1

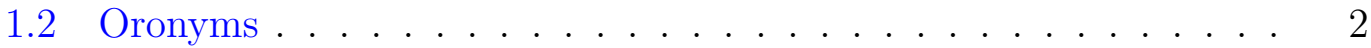

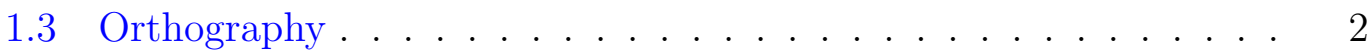

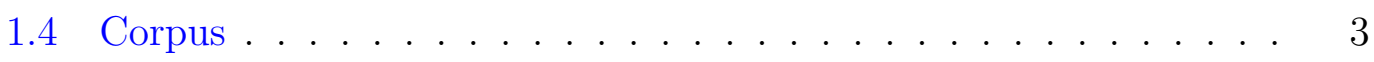

1.4.1 Uses of Text Corpora . . . . . . . . . . . . . . . 4

1.5 Word Categorizations . . . . . . . . . . . . . . . . . 4

1.5.1 Homographs ................. 4

1.5.2 Heterographs ................ 5

1.5.3 Homophones. . . . . . . . . . . . . . . . . 6

1.5.4 Homonyms . . . . . . . . . . . . . . . . . 6

1.6 Phonetics and Phonology . . . . . . . . . . . . . 6

1.6.1 Phonetics .................. 6

1.6.2 Phonology (aka phonemics) . . . . . . . . . 7

1.6.3 Phonetics Vs Phonology . . . . . . . . . . . . 7

1.7 Phonemic/Phonetic Alphabets . . . . . . . . . . . . . . 9

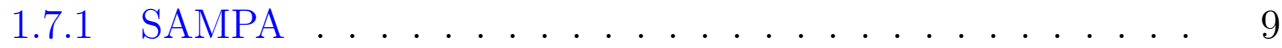

2 Introduction $\quad 11$

2.1 Why It Breaks Down . . . . . . . . . . . . . . . 11

2.2 Our Goals . . . . . . . . . . . . . . . . . . 13 
3 Implementation $\quad 15$

3.1 Customized Phonetic Dictionary . . . . . . . . . . . . . . . . . 17

3.1.1 Accent Choice . . . . . . . . . . . . . . . 17

3.1 .2 Dictionary Options . . . . . . . . . . . . 18

3.1.3 Custom dictionary fields . . . . . . . . . . . . . 20

3.1.4 Transferring the dictionary to a SQLite database . . . . . 21

3.2 Oronym Generation . . . . . . . . . . . . . . . . . 22

3.2.1 Step 1: Finding all phonemic variations of an orthographic phrase .................... . . 22

3.2.2 Step 2: Finding all Orthographic phrases for a Phonemic Sequence.................... 23

3.2.3 Word Frequency Evaluation . . . . . . . . . . . . 25

3.3 Visual Representation . . . . . . . . . . . . . . . . . 27

3.3.1 Oronym Tree Visualization . . . . . . . . . . . . . . 27

3.3.2 Oronym Sunburst Visualization . . . . . . . . . . . 33

4 User Study 46

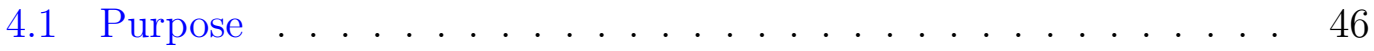

4.2 Structure . . . . . . . . . . . . . . . . . . . . 47

4.3 User Sampling Population . . . . . . . . . . . . . . . . 47

4.4 Methodology .......................... 48

4.4.1 First Phase: Recitation . . . . . . . . . . . . . . . 48

4.4 Recording Sample Pool . . . . . . . . . . . . . . . . 51

4.4.3 Second Wave: Transcription .............. 51

5 Results $\quad 53$

5.1 Phase One Results . . . . . . . . . . . . . . . . 53

5.2 Phase Two Results . . . . . . . . . . . . . . . . . 54

5.2.1 Transcribed oronyms' observed frequency vs. expected frequency ....................... 54

5.2.2 Statistical measurement of expected versus observed transcription frequency ............ 56

5.2.3 Observations on Transcription Count per Recording for each transcribed phrase . . . . . . . . . . . 59 
5.2.4 Transcription Breakdown By Country . . . . . . . . . 66

5.3 Deficiencies in The Oronyminator . . . . . . . . . . . . . 72

5.3.1 Frequency Validity ................ 72

5.4 Future Work . . . . . . . . . . . . . . . . 79

5.4.1 Higher-order frequency data . . . . . . . . . . . . . 79

5.4.2 Phoneme swapping . . . . . . . . . . . . . . . . 81

5.4.3 Melody Matcher master project . . . . . . . . . . 82

5.4.4 Target Audience and Goals . . . . . . . . . . . . . . 82

5.5 Conclusion . . . . . . . . . . . . . . . . 85

$\begin{array}{lr}\text { Appendices } & 86\end{array}$

$\begin{array}{ll}\text { A Implementation Details } & 86\end{array}$

$\begin{array}{ll}\text { B Oronym Tables } & 91\end{array}$

C SAMPA Phonetic Alphabet 105

$\begin{array}{ll}\text { D User Study Details } & 107\end{array}$

$\begin{array}{lr}\text { Bibliography } & 112\end{array}$ 


\section{List of Tables}

5.1 Phrase word frequency sum vs. times transcribed . . . . . . . 55

B.1 All Oronyms for 'A Nice Cold Hour' with frequency values . . . . 104 SAMPA Phonetic Alphabet . . . . . . . . . . . . . . . 104

C.1 Table of SAMPA Phoneme Makeup and Length . . . . . . . . . 105

D.1 Phrases Recorded . . . . . . . . . . . . . . . . 110

D.2 Countries and responses . . . . . . . . . . . . . . . 111 


\section{List of Figures}

1.1 Word Categorization Venn Diagram . . . . . . . . . . . 5

1.2 The difference between phonetics and phonology . . . . . . . . 8

1.3 Dictionary IPA screenshot $\ldots \ldots \ldots \ldots$

3.1 Geographic Origin of General American . . . . . . . . . . . 17

3.2 CMU dictionary entry example . . . . . . . . . . . . . . . . . 19

3.3 Custom dictionary entry example . . . . . . . . . . . . 20

3.4 Root oronym phrase . . . . . . . . . . . . . . . . . . 22

3.5 Tokenized root oronym phrase . . . . . . . . . . . . . 22

3.6 queryDBwithOrthoWordForSampa example . . . . . . . . . 22

3.7 Phonetic permutations of the ortho phrase "a nice cold hour" . . 23

3.8 Word Tree . . . . . . . . . . . . . . . . . . . . . . . . . . . . . 24

3.9 Phoneme to ortho graph of "a nice cold hour" . . . . . . . . . 26

3.10 IcedInkOronymsWithPartials _ . . . . . . . . . . . . 28

3.12 Seed Sphere vs Branch Radius Comparison . . . . . . . . . . . . 29

3.11 Freq Value For "Iced Ink" Oronym Words . . . . . . . . . . . 29

3.13 Unique First Words of "Iced Ink" Oronyms . . . . . . . . . . . 30

3.14 Dead end for oronym fragment Ice Ting . . . . . . . . . . . . 30

3.15 Success indicator sphere for complete oronym . . . . . . . . . . 31

3.16 Branch radius scaling to show frequency differences . . . . . . . 31

3.17 Oronym Phrases starting with aye . . . . . . . . . . . . . 32

3.18 Tail Phrases for aye . . . . . . . . . . . . . . . . . . 32

3.19 Oronym tree for the phrase iced ink . . . . . . . . . . . . 33 
3.20 Annotated oronym tree for the phrase iced ink . . . . . . . . . . 33

3.21 Annotated Oronym Parse tree generated for the phrase "fever pitch" 34

3.22 Example Sunburst Protovis data file . . . . . . . . . . . . . 36

3.23 Example Sunburst Diagram . . . . . . . . . . . . . . . 37

3.24 Equally-Weighted Sunburst Diagram for the oronyms of "iced ink" 39

3.25 Sunburst Diagram for the oronyms of "iced ink" weighted by UNISYN freq metric . . . . . . . . . . . . . . . . . . 40

3.26 Equally-Weighted Sunburst Diagram for the oronyms of "why that's insane" . . . . . . . . . . . . . . . . . 42

3.27 Sunburst Diagram for the oronyms of "why that's insane" weighted by UNISYN freq metric . . . . . . . . . . . . . . . . . . . 43

3.28 Equally-Weighted Sunburst Diagram for the oronyms of "an ice cold hour" . . . . . . . . . . . . . . . . . . . . . . . 44

3.29 Sunburst Diagram for the oronyms of "an ice cold hour" weighted by UNISYN freq metric . . . . . . . . . . . . . . . . . 45

4.1 Responses Per Country . . . . . . . . . . . . . . . . . 52

5.1 Most Common Transcriptions Globally . . . . . . . . . . . . . 55

5.2 Sunburst Chart for A Nice Cold Hour using UNISYN metrics for comparison to observed frequency sunburst . . . . . . . . . . . 57

5.3 Sunburst Chart for A Nice Cold Hour using observed frequencies . 58

5.4 Transcription Count Per Recording for the transcribed phrase "an ice cold hour" . . . . . . . . . . . . . . . . . . . . . . 61

5.5 Transcription Count Per Recording for the transcribed phrase "a nice cold hour" . . . . . . . . . . . . . . . . . . 62

5.6 Transcription Count Per Recording for the transcribed phrase "in ice cold hour" . . . . . . . . . . . . . . . . . . . . . . . . . . 64

5.7 Transcription Count Per Recording for the transcribed phrase "a nice gold hour" . . . . . . . . . . . . . . . . . 65

5.8 Transcription Count Per Recording for the transcribed phrase "a nice cold dower" . . . . . . . . . . . . . . . . . . . 67

5.9 Transcription Count Per Recording for the transcribed phrase "an eye scold hour" . . . . . . . . . . . . . . . . . . . . . . . . . 68

5.10 Transcription Count Per Recording for the transcribed phrase "an ice coal dower" . . . . . . . . . . . . . . . . . . 
5.11 Pie Chart of transcriptions from countries that are primarily English-

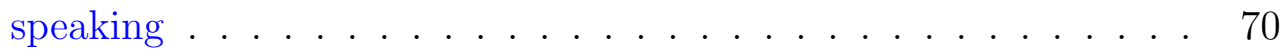

5.12 Pie Chart of transcriptions from countries that are Non-native English speakers . . . . . . . . . . . . . . 70

5.13 Bubble Chart comparison of Frequency for deer, does, and bucks . 74

5.14 Sunburst diagram for "an ice cold hour" using COCA by-document freq metric . . . . . . . . . . . . . . . . 76

5.15 Sunburst diagram for "an ice cold hour" using UNISYN freq metric 77

5.16 Historical N-gram data comparing the three-grams "a nice cold" and "an ice cold" . . . . . . . . . . . . . . . . . . 80

5.17 Historical N-gram data comparing the two-grams "a nice" and "an ice" . . . . . . . . . . . . . . . . . . . . 80

5.18 Historical N-gram data comparing the two-grams "nice cold" and "ice cold" . . . . . . . . . . . . . . . . . . . . . 81

A.1 Pseudocode for findAllPhoneSeqsForOrthoPhrase . . . . . . . 87

A.2 Pseudocode for discoverOronymsForPhrase . . . . . . . . . 88

A.3 Code for buildAndDrawFullTree . . . . . . . . . . . . . . . 89

A.4 Code for drawBranchesAtFork . . . . . . . . . . . . . . . . 90 


\section{Chapter 1}

\section{Preliminary Vocabulary}

Before we start, there are a few uncommon terms we will use fairly often in this paper. We have briefly defined them here.

\section{$1.1 \quad$ Mondegreens}

A mondegreen is a word or phrase resulting from a misinterpretation of a word or phrase that has been heard[11]. The word was coined by American author Sylvia Wright in her article, "The Death of Lady Mondegreen", published in a 1954 issue of Harper's Bazaar. In it, she describes the origin of the word:

When I was a child, my mother used to read aloud to me from Percy's Reliques, and one of my favorite poems began, as I remember:

Ye Highlands and ye Lowlands, Oh, where hae ye been?

They hae slain the Earl O' Moray, And Lady Mondegreen.

The fourth line of the quote is actually "and laid him on the green" [31]. 
Additional commonly-cited mondegreens include:

$$
\text { Gladly the Cross-Eyed Bear } \leftrightarrow \text { Gladly the Cross I'd Bear[7] }
$$

Scuse me while I kiss this guy $\leftrightarrow$ Scuse me while I kiss the sky [26]

There's a bathroom on the right $\leftrightarrow$ There's a bad moon on the rise [28]

\subsection{Oronyms}

Oronyms are phrases that may differ in meaning or spelling, but sound identical when spoken. They are similar to mondegreens, and the terms are often used interchangeably. The difference, however, lies in the context. The label "mondegreen" is used more often in regards to music lyrics, where pronunciation can be affected by the addition of music and tone to the phrase. Oronyms, on the other hand, refer to spoken words, not sung lyrics.[13] In addition, in this paper, the term oronym will refer only to phrases that are exact phonetic matches, whereas mondegreen will denote similar phrases with similar but not identical phonetics.

Common oronyms include:

$$
\begin{aligned}
\text { i scream } & \leftrightarrow \text { ice cream } \\
\text { an ice cold hour } & \leftrightarrow \text { a nice cold hour } \\
\text { grape ants } & \leftrightarrow \text { gray pants } \\
\text { real eyes } & \leftrightarrow \text { realize }
\end{aligned}
$$

\subsection{Orthography}

The word "orthographic" comes from the Latin orthographia, meaning correct writing. Orthography is the part of language study concerned with letters and spelling. More specifically, it is the standardized system of writing down words 
in a specific language, using a commonly-accepted set of letters according to accepted usage. [14]

The orthographic symbol set for a language is the commonly-accepted set of letters used to spell words in that language. In English, our orthographic symbol set is the Latin alphabet.

In this paper, "orthographic phrase" refers to a sequence of regularly-spelled words, as found in an English dictionary.

$$
\text { Example: "This is a orthographic phrase." }
$$

\subsection{Corpus}

The word corpus is Latin, and means body. In general, it is helpful to think of a text corpus as a "body of text" with some special constraints.

In linguistics, the term "corpora" refers to samples from various textual sources.

A "text corpus" refers to a large, structured body of text, consisting of those corpora (a.k.a. samples from various textual sources).

In order for a text corpus to be useful, it must be a representative subset of the larger language it wishes to represent.

To put together a general text corpus for the English language, one should pull from many sources: books, newspapers, movie scripts, magazines, academic literature, etc.

If any single genre is over-represented in the component corpora, then the 
resultant text corpus can be biased, and not useful for general purposes. For example, if one pulls all their corpora (text samples) from Wikipedia, the resulting text corpus is likely to underrepresent most first-person and second-person nouns and verbs, since those are forbidden in Wikipedia articles.

\subsubsection{Uses of Text Corpora}

A text corpus is generally used as the control set in linguistic experiments, allowing for experimental data to be measured against an expected result.

Given a well-sampled text corpus, word frequency can be generated simply by counting the number of occurrences of every word that appears in the corpus. This frequency data can be used by other applications, like MisheardMe Oronyminator, to weight the possibility of resolving homophones by observing which words have a higher frequency count in the corpus. The higher the frequency, the more common a word is, and the more likely it is to be heard.

In addition, given a text corpus, one can generate a dictionary of all words in the corpus. This dictionary can then be annotated with data such as: part of speech, unique identifiers for homographs, and phonetic spelling.

\subsection{Word Categorizations}

\subsubsection{Homographs}

Homographs are orthographic words that are spelled identically. Typically, homographs are also pronounced differently, which makes them homographic heterophones( 1.1). For example, there are two homographs for the word "does", 
because it has two different pronunciations: the "multiple female deer" does (doze), and the "third-person singular present indicative form of 'do' " does (duhz). If the words are spelled identically AND pronounced identically, then they are homographic homophones, which are commonly known as homonyms.

\subsubsection{Heterographs}

Heterographs are orthographic words that are spelled differently. Typically, words are only called heterographs if they are also pronounced differently, making them heterographic homophones. Most words in the English language are heterographic heterophones; that is, spelled and pronounced uniquely. Because this is the default state of a word set, we rarely describe such words in terms of homo/ hetero phones/graphs. As such, the only time a word set is likely to be described as heterographic is if it is

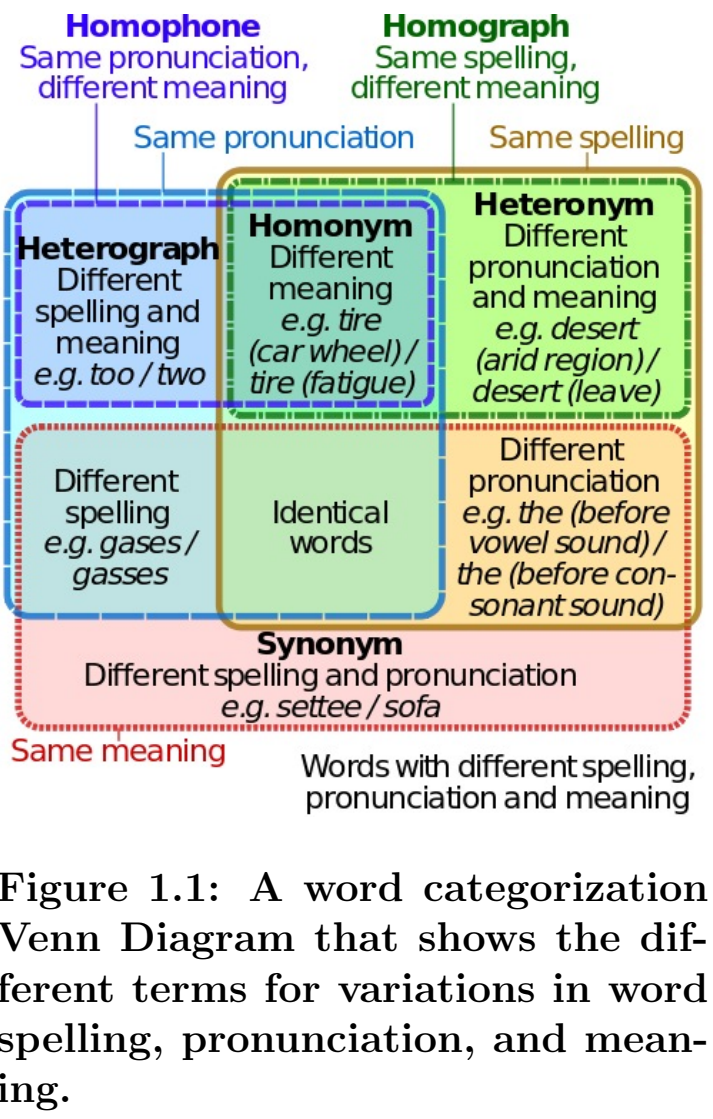
also a homophone. For example, the banes of every grammarian, "there", "their", and "they're", are heterographic homophones. Alternatively, "to", "too", and "two" are also heterographic homophones. 


\subsubsection{Homophones}

Homophones are orthographic words that are pronounced identically, but typically spelled differently. If a homophone set is also homographic (that is, spelled identically as well as pronounced identically), then we refer to them as homonyms. As such, the only time the words in a set are likely to be labelled "homophones" is if they are also heterographic.

\subsubsection{Homonyms}

Homonyms are orthographic words that are pronounced and spelled identically, but defined differently. For example, depending on context, the word "left" can mean left (the opposite of right), or left (the past tense of leave). Homonyms can also be referred to as homographic homophones.

\subsection{Phonetics and Phonology}

To discover oronyms for a phrase, we must first translate the root orthographic phrase to a representation that allows us to unambiguously measure pronunciation. Phonology and phonetics are branches of linguistics that deal with pronunciation.

\subsubsection{Phonetics}

Phonetics is a branch of descriptive linguistics, and refers to the study of the actual, uttered sound of human speech. It deals with describing the physical phenomena of how these sounds are produced from the vocal tract, how they are 
transmitted once spoken, and how they are recieved by audiences. The building blocks of phonetics are phones, which represent atomic sounds.

\subsubsection{Phonology (aka phonemics)}

Phonology is a branch of theoretical linguistics, and as such, is primarily concered with the abstract grammatical characterization of sounds. It describes the way that sounds function within a language to give meaning to words. The basis of phonological analysis is the grouping of sounds (phones) into distinct units within a language. These distinct units are called phonemes.

These phonemes may contain different phones, depending on the accent of the speaker. For example, native speakers of General American English only generally recognize one 'L' sound phoneme. However, there are two different ways that that phoneme manifests itself: the 'l' in "male", and the 'l' in late. This difference is not noticeable to a native speaker of American English, because that particular accent will parse any ' $L$ ' phone as the same ' $L$ ' phoneme. It would, however, be recognizable to someone whose accent categorizes those phones into two separate phonemes.

\subsubsection{Phonetics Vs Phonology}

Though the terms are sometimes used interchangably, the words "phonemic' and 'phonetic' (and their corresponding sound building blocks, 'phone' and 'phoneme') indicate different stages of sound parsing. Phonemes are idealized sounds; phones are the actual sounds that come out of a person's mouth. Figure 1.2 provides a final, illustrative metaphor of the difference. 


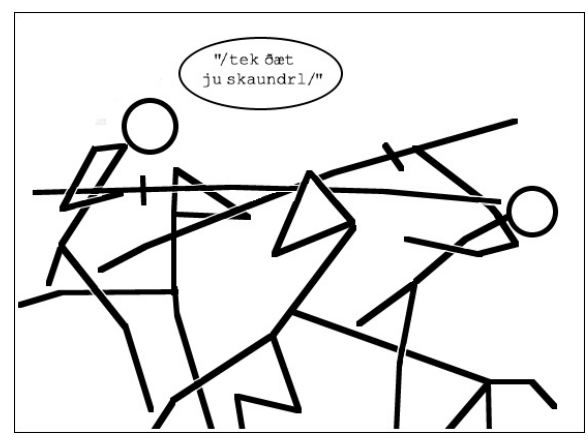

(a) Phonology $[8]$

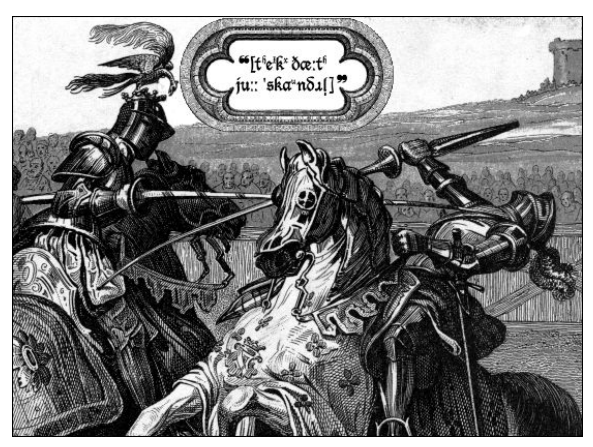

(b) Phonetics[9]

Figure 1.2: The difference between phonetics and phonology 


\subsection{Phonemic/Phonetic Alphabets}

As we stated in section 1.6.2, phonemes are the atomic building blocks of words. In a phonemic alphabet, every meaningful sound has its own "letter". The way that we interact with phonemes in a concrete, textual way is by using phonetic alphabets and phonetic dictionaries.

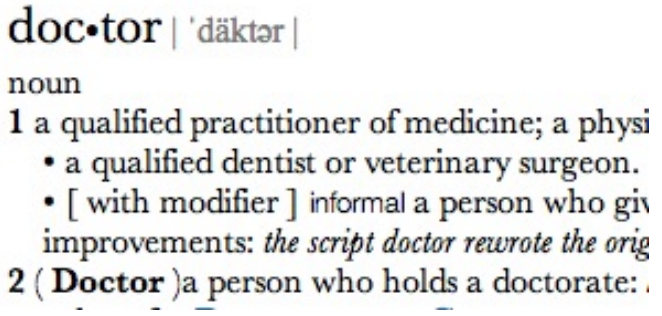

Figure 1.3: The characters to the right of the large bold word "doctor" are IPA symbols.

The most common phonetic alphabet is the IPA (International Phonetic Alphabet). It contains representations of every sound in every known language globally, and allows for cross-cultural pronunciation guidelines. As shown in figure 1.3, IPA representations of orthographic words are found in traditional dictionaries to aid pronunciation.

\subsubsection{SAMPA}

SAMPA (Speech Assessment Methods Phonetic Alphabet) is a computerreadable phonetic alphabet, based upon the symbols found in the more-standardbut-not-easily-computer-readable IPA (International Phonetic Alphabet). It uses "letters" consisting of 1-2 ASCII characters to represent each phoneme. The ASCII sequences corresponding to each of the SAMPA letters are designed so that any SAMPA sequence is deterministically parsable.

We chose to use SAMPA instead of IPA because its ASCII-compliance makes it easy to integrate into other systems. 
See the table in Appendix $\mathrm{C}$ for a full table of each SAMPA phoneme, its description, and its sub-parts.

For some brief examples, the SAMPA spelling of the name 'Jenee Hughes' is dZEni hjuz. 'Dr Zoe Wood' becomes dAkt@ 'r zoui wUd. 'Dr John Clements' becomesdAkt@ ‘rdZAn klEm@nts. 'Dr Franz Kurfess' becomes dAk@ ‘r fr\{nz k3`rfEs. 


\section{Chapter 2}

\section{Introduction}

Human brains are built to come to single conclusions about ambiguouslywritten, -spoken, or -heard text. Those single conclusions are developed based upon one's experiences, liguistic expectations, cutural immersion, and language familiarity [29]. When attempting to write English phrases that will be read aloud and heard by people with different linguistic biases, it is important to make prose as deterministically understandable as possible. The first step towards this is understanding and identifying how many ways a particular textual phrase can be misheard, and why.

\subsection{Why It Breaks Down}

There are two points at which an author's intendeded interpretation of prose written to be performed for an audience can be muddled: First, when the author's orthographic text becomes an orator's spoken (phonetic) interpretation, and second, when the orator's phonetic interpretation is translated phonetically by an audience into a perceived orthographic phrase. Both of these interpreta- 
tions must be made succesfully in order for the author's original intended meaning to be conveyed.

The phrase "iced ink" undisputedly succeeds in the first translation from orthographic phrase to phonetic interpretation, since there is only one possible pronunciation. However, it fails on the second. "Iced ink" can only be pronouced one way, but it can be heard multiple ways-the most notable of which is "I stink", not "iced ink".

The phrase "a nice cold hour" can fail at both junctures of interpretation. First, the orator could have accidentally capitalized the word Nice in their head, and made it sound like Nice, the city in France. An audience would likely hear this as "niece", and would be confused, at best. Even if the orator pronounces the phrase as the author intended, the audience could hear multiple orthographic phrases in the same phonetic sequence: "a nice cold hour", "an ice cold hour", or even "a nigh scold our".

A third, more rare and nefarious type of audience misunderstanding can be caused by parse-tree misdirection, where an audience member is absolutely sure they are hearing one phrase, only to get lost halfway through the phrase because they were interpreting a phonetic sequence in a way that resulted in an orthographic dead end. This happens due to the relative frequency of the possible words heard in the aurally-interpreted phrase.

A good example of the effects of this phenomenon can be found when looking at listeners' incidental memorization of popular song lyrics. When asked to sing along with the Adele song, Rolling in the Deep, people who were singing enthusiastically at the start dropped out around the line "reaching a fever pitch" [20]. Let us consider the phrase "fever pitch". This phrase has no exact oronyms, but 
it does have a potential dead end- a listener could hear the first syllable of the phrase as the word "fee", which has a frequency of 7265. That is more than double the frequency of the word "fever", which is 3095. Since the human brain is predisposed to parse more-familiar words, having that heavily-weighted deadend branch is likely the cause of the casual listener not being able to effortlessly memorize the correct lyrics by ear[27].

\subsection{Our Goals}

All of the problems in Section 2.1 can be solved by oronym analysis, by comparing phrase interpretation probabilities, evaluated by using word frequency values. To this end, we aim to demonstrate a technique used to validate word frequency dictionary values. We specifically chose to evaluate frequency values from the UNISYN dictionary, which tallies each word on a per-occurance basis, using a proprietary text corpus, to calculate word frequency.

In the first phase of our user study, we generated oronym strings for the phrase "a nice cold hour", and had over a dozen people make 62 recordings of the most-common oronyms for that phrase. In the second phase, we selected 15 of the phase one recordings, and had approximately 74 different people transcribe each one, for a total of 953 transcriptions overall.

If the UNISYN frequency dictionary values for our test phrases had accurately reflected the real-world expectations of actual listeners, we would expect that the most-commonly transcribed phrases in our user study would roughly correspond with our metric for the most likely oronym interpretation of the root phrase.

During the course of our study, we found that using per-occurance frequency 
values, like those found in the UNISYN dictionary, when computing our overallphrase-frequency metric caused the end result to be thrown off by excessively common words, such as "the", "is", and "a". These super-common words had such high per-occurance tallies that they overpowered any effect that any regular word had on a frequency metric. When we used frequency values from the COCA dictionary, which has word frequency values tallied on a document-count basis instead of a UNISYN-like per-occurance basis, we found that this effect was mitigated. As a result, we do not recommend using the UNISYN dictionary for word frequency purposes. 


\section{Chapter 3}

\section{Implementation}

We present a computer program that takes in a textual phrase in English, determines all oronyms for that phrase, and then visualizes those oronyms in two ways: in tree form, with branch width scaled by word frequency metrics to indicate the likelihood of interpretation; and in sunburst diagram form, where all valid oronyms radiate from a center root, with each arc scaled by frequency.

To accomplish this, the program has four major functional parts: a custom phonetic dictionary, a command-line oronym generator, an OpenGL oronymparse-tree visualization generator, and a Protovis datafile generator.

We compiled the custom phonetic dictionary using data from the UNISYN Lexicon. Then, our custom phonetic dictionary is used by command-line oronym generator, our oronym-parse-tree visualization generator, and our Protovis datafile generator. These three components are run independently of each other, and produce independent output, but they are all based on the same $\mathrm{C}++$ code base.

To get a simple textual list of oronyms, a user feeds a textual phrase into the command-line oronym generator. The command-line oronym generator then 
looks up the possible SAMPA interpretations of the words in the phrase supplied by the user, and compiles all possible phonetic permutations. Then, it goes through each possible SAMPA sequence, and finds all possible complete orthographic interpretations for that particular phonetic sequence. After it has found all possible complete orthographic interpretations of all phonetic (SAMPA) permutations of the original input phrase, it deduplicates the list and returns a this list of oronyms to the user.

To use the oronym-parse-tree visualization generator, the user supplies a phrase that they want to create an oronym parse tree for. Once again, the program finds all possible SAMPA phonetic sequences of the supplied phrase. Then, it compiles all complete and all partial orthographic interpretations of those phonetic sequences. These complete and partial oronym phrases are examined for commonalities, and then a rough tree data structure is created that keeps track of all orthographic divergences. Following this tree, the program queries the custom phonetic dictionary for word frequencies, which it uses to scale branch radii in the visualization it then draws.

To use the last part of our implementation, the Protovis datafile generator, the user once again inputs the phrase they want to analyze the oronyms of. The datafile generator discovers all the oronyms using the same process that the command-line oronym generator does. Then, it looks up the word frequencies of all the component words of the oronyms generated. It then outputs a .js file that plugs into an HTML skeleton. The user clicks on the HTML file after running the Protovis datafile generator, and the interactive sunburst diagram is displayed 


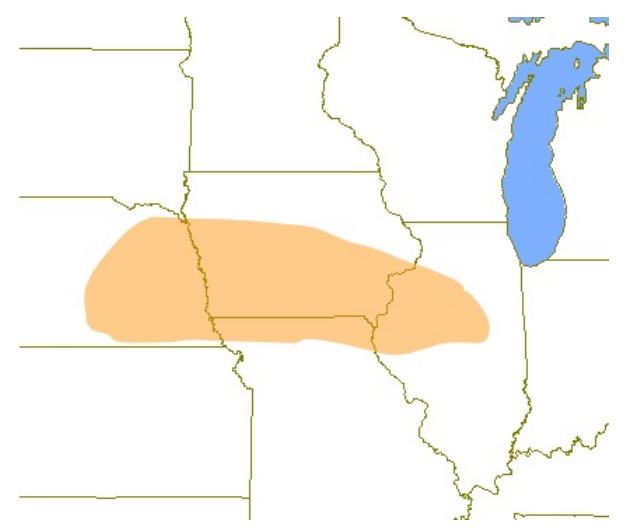

Figure 3.1: This is the geographic area whose accent most closely resembles the General American Accent [6]

\subsection{Customized Phonetic Dictionary}

In order to discover oronyms for each phrase, we first needed to determine how each phrase is pronounced. Pronunciation can vary depending on the speaker's accent, so it was important for us to (1) choose an accent that we could easily replicate and (2) find a dictionary that supported that accent.

\subsubsection{Accent Choice}

We decided to utilize a General American accent, due to its ubiquity in media and news sources. The General American accent, also known as the "Standard American English" dialect, is not spoken by most Americans, but is used as an "average accent". It most closely resembles the Midwestern accent used in the area in Figure 3.1, but is more commonly recognized as "the newscaster accent". Newscasters learn this accent for use on national TV, because it is the "leastaccented" of the American accents[19].

The downside of using the General American accent is that, while it does give a good approximation of most American's speaking accents, it does not 
perfectly reflect a "singing accent". Singers tend to elongate syllables, changing emphasis placement in words, and vowels tend to be sung in a more "round" matter[22].For example, though the dictionary pronunciation of the word "baby" is "be $\$$ bi (bay-bee), in songs, you commonly hear the pronunciation "be $\$$ be (bay-bay). The e sound is easier to sing than the i (ee) sound, because the latter requires the singer move their mouth and vocal cord position further from neutral than the former does[16].

However, since different singers will change pronunication for different vowels based upon which vowels are easiest for them to sing, no definitive pronunciation guidelines or rules exist. Therefore, using the General American accent gave us as good an approximation as we were likely to get[18].

\subsubsection{Dictionary Options}

We considered using three different phonetic dictionaries: the CMU dictionary, LC-STAR dictionary, and UNISYN dictionary[10] [2] [17]. We started out by looking at the LC-STAR dictionary, but quickly decided that it was not going to be as useful to us, because the LC-STAR dictionary is not particularly well-maintained.

The CMU dictionary showed promise, but had a few shortcomings. In its favor, it had a very simple way of encoding words: first the orthographic word, then the identifier number in parentheses (if needed), then a space, then a oneto-two char code for each sound in the word, with the numbers $0,1,2$ appended to indicate emphasis (if needed), separated by spaces. An example of a CMU dictionary entry can be seen in Figure 3.2.

The problem that arose with this format was that there was no explicit defini- 


\section{Figure 3.2: Here is the CMU dictionary entry for the word "abbrevi- ate"}

tion of where to hyphenate the word when splitting it up. This causes problems for words in song lyrics, where each note has its own syllable underneath it, and each syllable might have many different sounds. In addition, it used nonstandard symbols for its phonetic alphabet, which would complicate matters if, in the future, we chose to combine data from other dictionaries with our existing dictionary. Most importantly, the phonetic sequences, if the spaces were removed, would not be deterministically parsable.

The UNISYN dictionary is used primarily to phonetically translate words into multiple accents. It has its own dictionary entry format, with a series of wildcards representing different phones. UNISYN provides some semi-functioning perl scripts that allow a user to specify a dialect they would like to use (For example, a Californian would say "cooking" differently than someone from the Deep South, and both would say it differently than someone from London. However, they are all speaking English. The UNISYN dictionary facilitates this translation).

The UNISYN dictionary had all the information we needed, and then some. However, it was case-insensitive, meaning that it did not make it easy to differentiate pronunciations for some words. For example, the word "nice" is pronounced differently from the city "Nice", but they were both stored as "nice" in the orthography of UNISYN. This was a minor setback, but we were able to design our user study around this limitation, and ultimately decided to use the UNISYN dictionary exclusively. 
Example:

transfer : $2:$ VB/VBP $: \operatorname{tr}\left\{\mathrm{ns}^{\prime \prime} f 3^{\prime} r: \operatorname{tr}\left\{\mathrm{nsf} 3^{\prime} r: \quad\{\right.\right.$ trans==fer $\}$

: 7184

Figure 3.3: Here is an example of an entry in our custom phonetic dictionary, using the word "transfer"

\subsubsection{Custom dictionary fields}

Below we lay out the format for the fields in an entry of our custom phonetic dictionary, which we generated using the UNISYN dictionary's perl script output for the General American accent:

$<$ ortho $>$ : <uniqueID $>$ : < partOfSpeech $>$ : $<$ SAMPAspelling $>$ :

$<$ SAMPAnoEmph $>$ : <extendedOrtho $>$ : $<$ freq $>$

$<$ ortho $>$ is the regular, orthographic spelling of the word.

$<$ uniqueID $>$ is a number (and optional string) used to differentiate homographs $^{2}$.

$<$ partOfSpeech $>$ is used to identify the specific part of speech for the word.

$<$ SAMPAspelling $>$ is the breakdown of the word, phonetically. It uses the SAMPA alphabet, and separators to show where breaks in the word are, and how they are emphasized. If a separator is $\$$, the subsequent phones (until the next separator) are not emphasized. If it's \%, then they are pronounced using secondary emphasis. If it's ", then they are given the primary emphasis in the word.

\footnotetext{
${ }^{2} \mathrm{~A}$ homograph shares the same written form as another word but has a different meaning; For example, a farmer would sow (verb) seeds in a field, but could also raise a sow (noun) for bacon.
} 
$<$ SAMPAnoEmph $>$ is the same as $<$ SAMPASpelling $>$, but with all emphasis separator characters stripped out. We chose to add this field so that we could more easily look up phonetic sequence matches.

$<$ extendedOrtho $>$ allows for stemming analysis of words, for possible use in future work.

$<$ freq $>$ is the frequency at which the word occurs in language, according to UNISYN. The frequency count is "taken from a composite of a number of on-line sources of word-frequency. It includes frequencies from the British National Corpus and Maptask, and frequencies derived from Time articles and online texts such as Gutenberg. They were weighted to give more importance to sources of spoken speech, and also to increase the numeric frequency of smaller corpuses" [25].

An example of a entry in our custom phonetic dictionary can be seen in Figure 3.3.

\subsubsection{Transferring the dictionary to a SQLite database}

Because there are several hundred thousand entries in our phonetic dictionary, it was necessary to have a database, rather than store them all in-program in a multi-dimensional array. We decided to use a SQLite database for this purpose.

To turn the colon-delimited dictionary text file into a SQLite database, we decided to use a program called the "SQLite Database Browser", an open source,

public domain, freeware visual tool to create, design, and edit SQLite3.x database files. We specifically used version $2.0 \mathrm{~b} 1$ of the program, which was built with version 3.6.18 of the SQLite engine[15]. 


\subsection{Oronym Generation}

\subsubsection{Step 1: Finding all phonemic variations of an ortho- graphic phrase}

First, our program takes an orthographic phrase to find oronyms for (Figure 3.4).

$$
\text { 'a nice cold hour' }
$$

Figure 3.4: A valid orthographic phrase

We then tokenize this phrase into its component words, using whitespaces as a delimiter (Figure 3.5).

$$
\text { 'a', 'nice', 'cold', 'hour' }
$$

Figure 3.5: The root orthographic phrase, tokenized

We query our phonetic dictionary for all possible SAMPA pronunciations (Figure 3.6).

$$
\begin{gathered}
\text { 'a' } \rightarrow \text { e, @, A } \\
\text { 'nice' } \rightarrow \text { naIs, nis } \\
\text { 'cold' } \rightarrow \text { kould } \\
\text { 'hour' } \rightarrow \text { aU'r }
\end{gathered}
$$

Figure 3.6: In this and all subsequent diagrams, a 'string in quotes' indicates an orthographic word or phrase, and a monospaced string indicates that it is a SAMPA word or phrase.

Now that we have the pronunciation of each of the words in the form of 
SAMPA strings, we can list all the possible phonetic permutations of the original phrase(Figure 3.7).

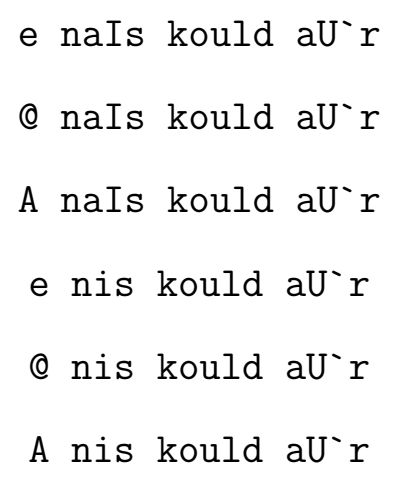

Figure 3.7: Phonetic permutations of the ortho phrase "a nice cold hour"

The pseudocode for this process can be reviewed in Figure A.1.

\subsubsection{Step 2: Finding all Orthographic phrases for a Phone- mic Sequence}

Then, for each phonemic phrase, we want to figure out all valid orthographic interpretations. For this, we have to go back to our phonetic dictionary.

The ideal way to think about searching for words in a phonetic sequence is by picturing the phoenetic sequence in a tree form, similar to the tree pictured in abbreviated form in Figure 3.8. For example, if I had a phonetic tree with the entire dictionary in it, each phonetic tree node would have at least 45 child nodes: one for each phone. A node might also have "word" child nodes, if the phones along the path to that node construct a valid orthographic word.

When there are multiple orthographic interpretations at a single phonetic node, the most likely interpretation can be determined by checking the frequency 
of use for each word. For example, the sequence $\mathrm{n}$ aI $\mathrm{s}$ is much more likely to be "nice" than "gneiss". Figure 3.8 shows a visual representation of traversing an entire dictionary's phonetic tree for nodes along the paths for the SAMPA sequences aI $\mathrm{s}$ and $\mathrm{n}$ aI $\mathrm{s}$.

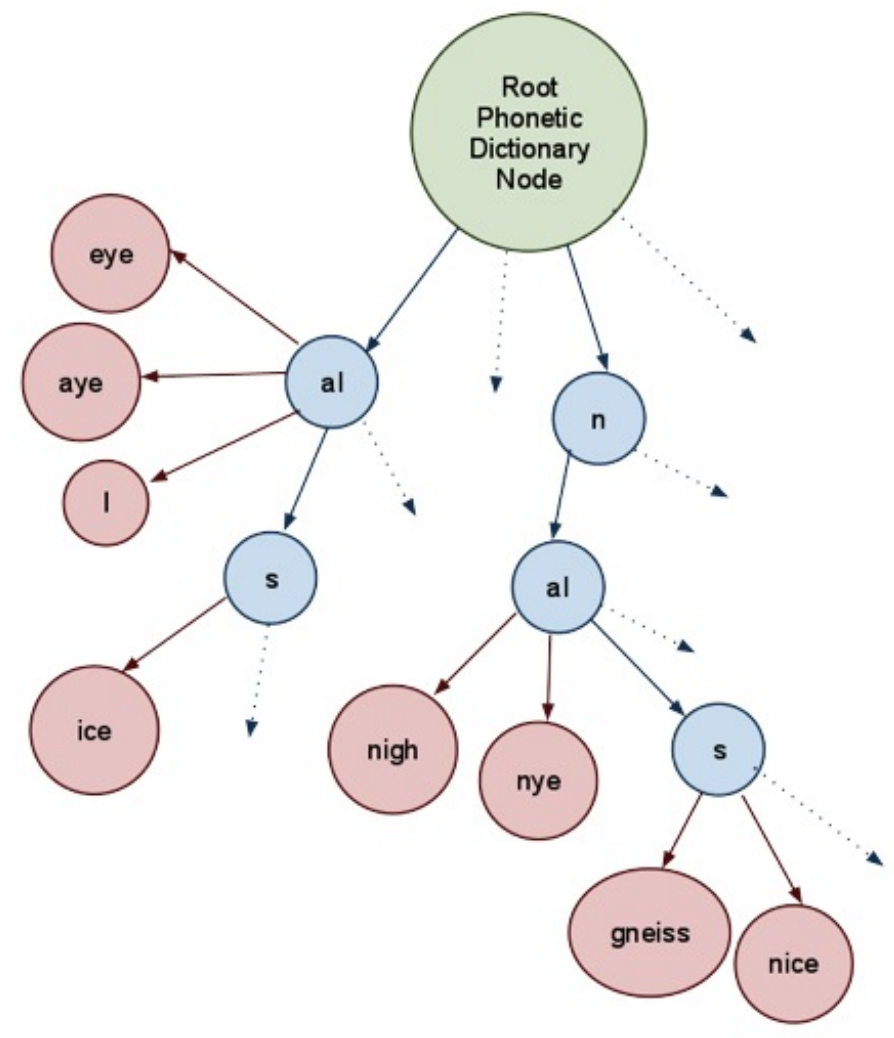

Figure 3.8: Word tree

We can use this dictionary tree method to discover all valid orthographic interpretations for any phonetic sequence of our root orthographic phrase, as shown in Figure 3.9 for the phrase "a nice cold hour".

Once we have grabbed all the orthographic interpretations for each phonetic sequence, we combine them all into an orthographic oronym phrase list. This process may leave us with some redundant oronyms, so we deduplicate that list.

After this, we have a list of all unique and valid oronyms for the original root 
phrase.

In the case of "a nice cold hour", this returns 290 oronyms, as seen in the first column of Table B.1.

The pseudocode for this process can be reviewed in Figure A.2.

\subsubsection{Word Frequency Evaluation}

Next, we want to evaluate all our oronyms based on how common each oronym's component words are. For example, "a nice cold hour" is much more likely to be heard than "a gneiss cold hour," even though both are phonetically identical.

To evaluate oronym frequency comparatively, we tokenize each oronym phrase into its component words, once again delimiting by non-newline whitespaces.

Then, we query our phonetic dictionary with each word to get that word's frequency value. We store each word's value separately. When we have retrieved the frequencies for all the words in a phrase, we then sum up all the frequencies to give a combined frequency of the entire phrase.

You can see these frequency counts for each word in all oronyms of the phrase "a nice cold hour" in Table B.1. 


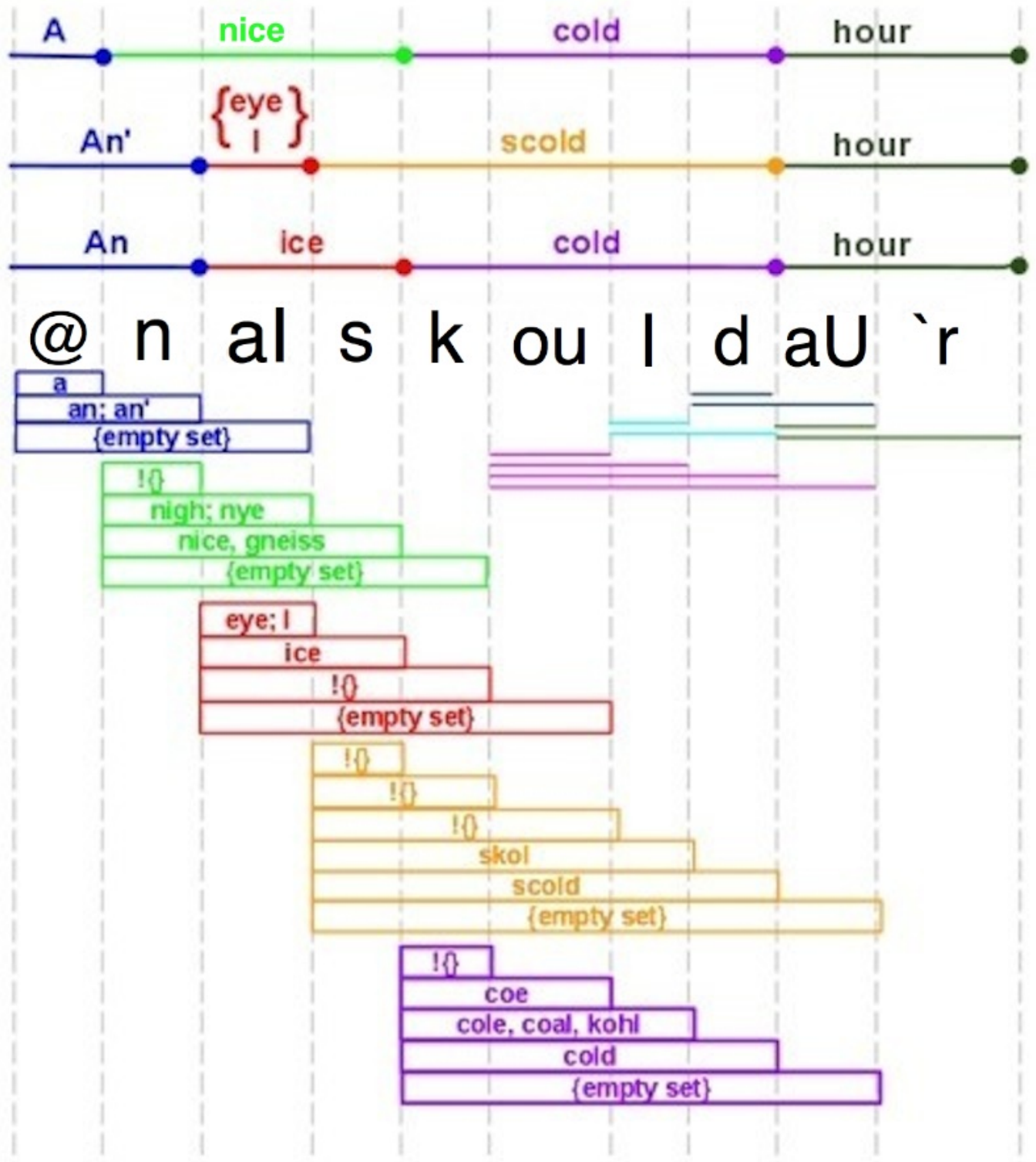

Figure 3.9: Phoneme to ortho graph of "a nice cold hour" 


\subsection{Visual Representation}

We created two different oronym visualizations. The first, oronym trees, were chosen for their ability to show the phonetic dead ends that may happen during oronym aural interpretation. Our particular oronym tree visualization is written in $\mathrm{C}++$ using OpenGL, which allows for future integration into any other $\mathrm{C}++$ codebase.

The second visualization uses sunburst diagrams to show all valid oronyms of a root phrase. These sunburst diagrams were created using the ProtoVis library, and use javascript data files with html wrappers. The javascript data files were generated using the same $\mathrm{C}++$ code that the first visualization used, so both visualizations show identical data. However, the oronym sunburst diagrams more easily exhibit the weighting of the different oronym paths with respect to their frequency dictionary values.

\subsubsection{Oronym Tree Visualization}

We go about building the visual representation of the oronym parse tree in much the same way that we build the textual list of oronyms, with one important caveat: our oronym parse trees may contain incomplete oronym strings, or oronym fragments. Oronym fragments occur when there is a valid orthographic interpretation of a leading subsequence of a phonetic sequence for which the remaining phonemes in the sequence do not map to any valid orthographic word or phrase. For example, for the root oronym phrase "iced ink" (aI s t I N k), a listener may hear and interpret up to "I sting" (aI s t I N), and then be confused when the last phone, $\mathrm{k}$, comes along. 
Our algorithm for building the tree diagram is recursive, called from a parent function that draws the tree's 'seed' sphere. This parent function engages in a depth-first traversal of the oronym tree, and is documented in Figure A.3.

We start in the parent function by getting all the oronyms of our orthographic phrase, using the process outlined in sections 3.2.1 and 3.2.2. However, instead of ignoring any incomplete orthographic interpretation of a phonetic sequence, as we do in

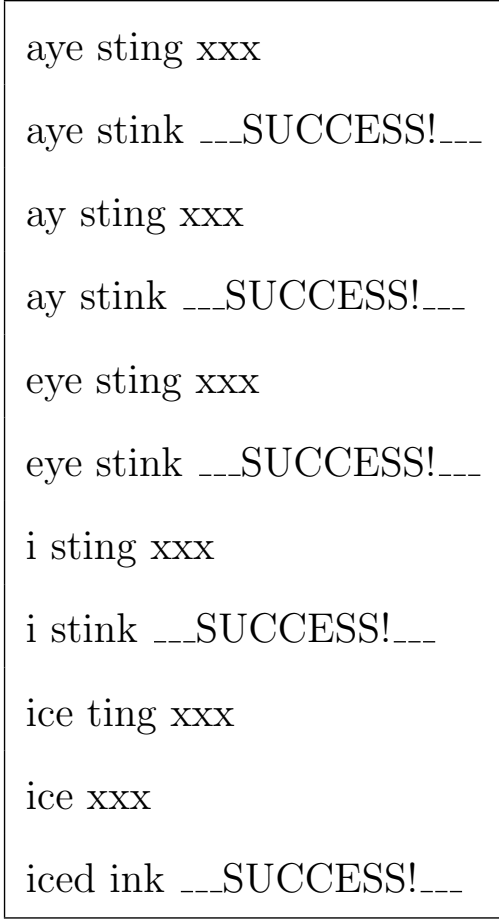

Figure 3.10: All partial and complete oronyms for the phrase "iced ink"

section 3.2.2, we add the incomplete

phrases to the list of oronyms, keeping

track of them by appending 'xxx' or 'fff' to the end of each incomplete oronym string. For example, as shown in Figure 3.10, the phrase "iced ink" may only have five complete oronyms, but it has six additional oronym fragments, making for 11 possible interpretations.

Next, we tokenize our generated phrases by whitespace, and look up the frequency of each word, as shown for "iced ink" in Figure 3.11. We will later scale our branches' radii using the maximum and minimum word frequency values found during this run. In this case, the maximum frequency is $9,937,877$ for the word "I", and the minimum is 124 for the word "ting". 


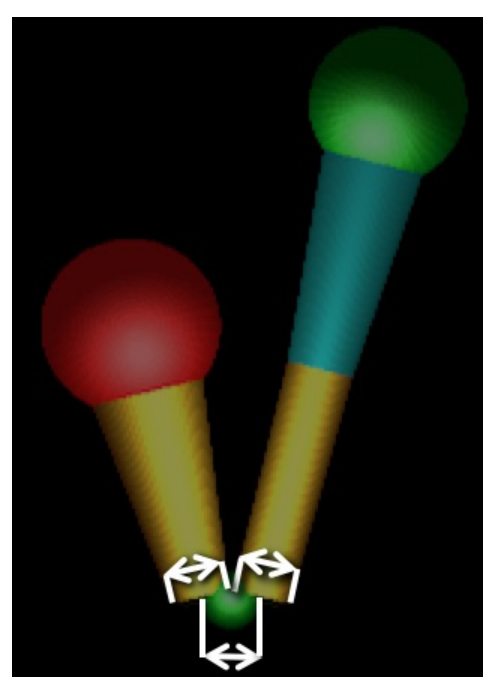

Figure 3.12: Seed sphere vs branch radius comparison

\begin{tabular}{|l|} 
aye $=130503$ \\
ay $=6633$ \\
eye $=26750$ \\
$\mathrm{i}=9937877$ \\
ice $=12202$ \\
iced $=402$ \\
sting $=1472$ \\
ting $=124$ \\
ink $=2589$
\end{tabular}

Figure 3.11: Frequency values for all unique words in "iced ink" oronyms
Once we have all partial and complete oronyms, plus the maximum and minimum word frequency values found in all those phrases, we pass them into our recursive function, along with the radius of the seed sphere. That seed radius will be the beginning radius of each root-level branch, as shown in Figure 3.12

Inside our recursive function, we pull the first word out of each orthographic phrase, and create a set of unique first words, as seen in Figure

\subsection{3}

We then go through this set of unique first words iteratively. 


\begin{tabular}{|l|l|l|l|l|l|}
\hline aye & ay & eye & i & ice & iced \\
\hline
\end{tabular}

\section{Figure 3.13: All unique first words of "iced ink" oronyms}

For each word, we look up frequency in the phonetic dictionary. Then, we use the maximum and minimum frequencies that we found in our parent function, plus constants for maximum and minimum radius size, to scale that frequency into a usable radius size.

Then, we check the contents of the word string.

If the word is "xxx" or "fff", then it is not a word at all-just an indication of the dead end of an oronym fragment. In this case, as seen in Figure 3.14, we draw a red sphere with the radius of the branch's ancestor, using the radius parameter passed into our recursive function for 'lastRadius'.

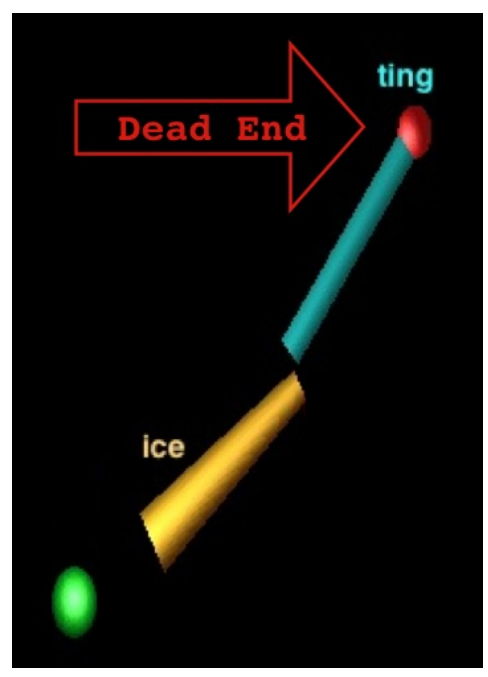

\section{Figure 3.14: Dead end sphere for oronym fragment "ice ting"}

If the word is "_-_SUCCESS!_-_", that indicates a full oronym has been successfully found, and is terminating at that point. A green sphere is drawn at the end of that branch, using the lastRadius parameter for branch radius size, as seen 
in Figure 3.15 for the phrase "I stink".

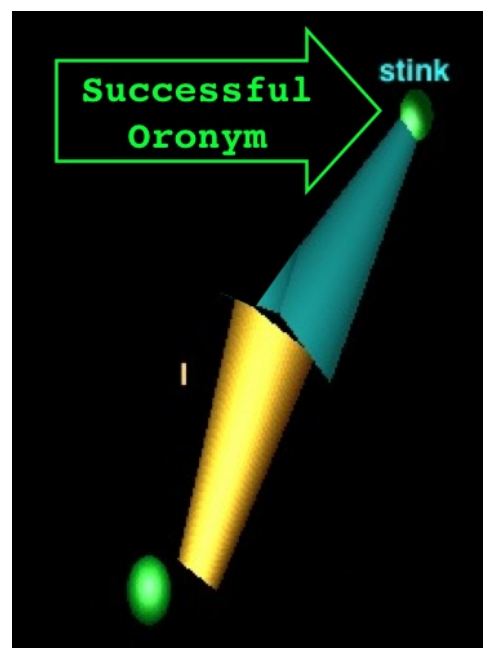

Figure 3.15: Success indicator sphere for complete oronym "I stink"

If the word is not "xxx", "fff", or "_-_SUCCESS!_--", it is a valid orthographic word, and we draw a cylinder "branch" representing that word. The cylinder's bottom radius is equal to lastRadius, and the top radius is equal to the scaled radius that we derived using the word's frequency. An example of this branch radius scaling is show in Figure 3.16 .

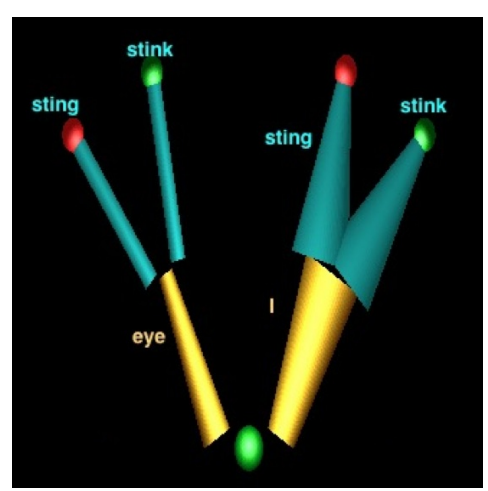

Figure 3.16: Scaled branch radii showing frequency difference

After we draw the cylinder, we then go through the full list of phrases, and compile a list of all phrases that start with the word we just drew the cylinder 
for, as in Figure 3.17. Then, we remove the first word from each of those phrases, deduplicating the resulting list of "tail" phrases, which is shown in Figure 3.18.

\begin{tabular}{c}
\hline aye sting $\mathrm{xxx}$ \\
aye stink _-_SUCCESS!_--- \\
\hline
\end{tabular}

\section{Figure 3.17: All oronym phrases of "iced ink" starting with "aye"}

\begin{tabular}{|l|}
\hline sting $\mathrm{xxx}$ \\
stink __-_SUCCESS!
\end{tabular}

Figure 3.18: Tail phrases for oronyms of "iced ink" that begin with "aye"

Then, we change our material color (so that different levels of branches will be different colors), and make a recursive call to our current function, passing as parameters the scaled radius and the list of tail phrases.

After this recursive call, we change our color material back to whatever it was before the call, and then continue on to the next unique first word in our set, which, in this case, is "ay".

Once we have looped through all our unique first words, we know we are done drawing that set of branches, and we return.

This gives us the oronym parse tree seen in Figure 3.19. As shown in Figure 3.20 (the annotated version of Figure 3.19) each branch on the tree represents a single orthographic word.

Another example of an oronym fragment phrase can be found when looking at the oronym parse tree for the phrase "fever pitch" in Figure 3.21. The branch for "fever" ends in a much smaller radius than the branch on the left for the word 
"fee". As you can see by the relative size of the end spheres of the branches, the word "fee" even outweighs the last word in the other branch as well (which is "pitch", with a frequency of 5104). This indicates that a listener is very likely to hear the oronym fragment over the complete oronym.

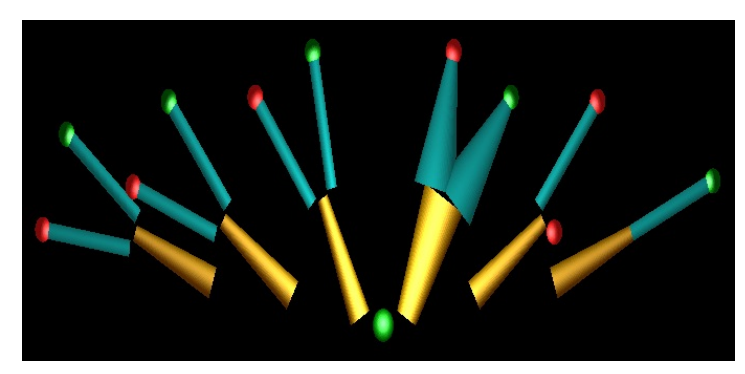

Figure 3.19: Oronym tree for the phrase "iced ink"

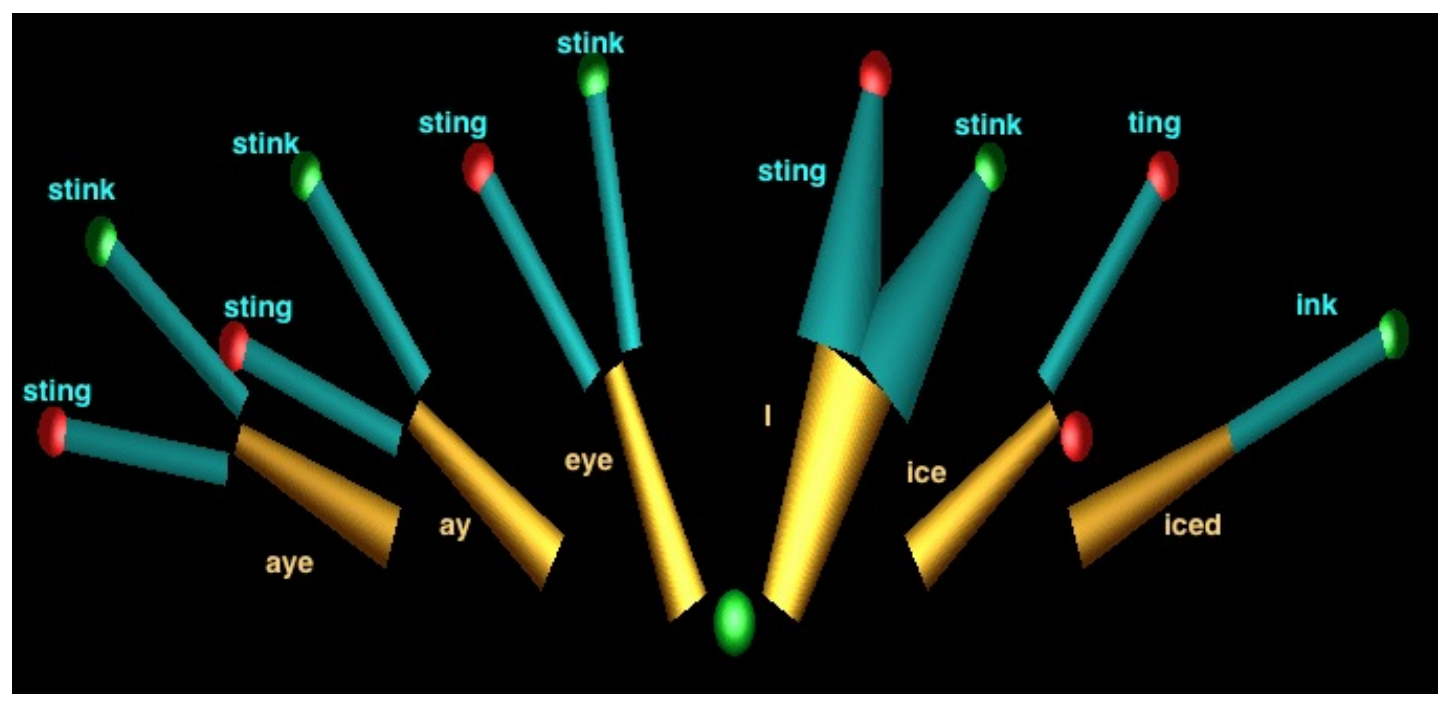

Figure 3.20: Annotated oronym tree for the phrase "iced ink"

\subsubsection{Oronym Sunburst Visualization}

For our second visualization type, we chose to use sunburst diagrams. 

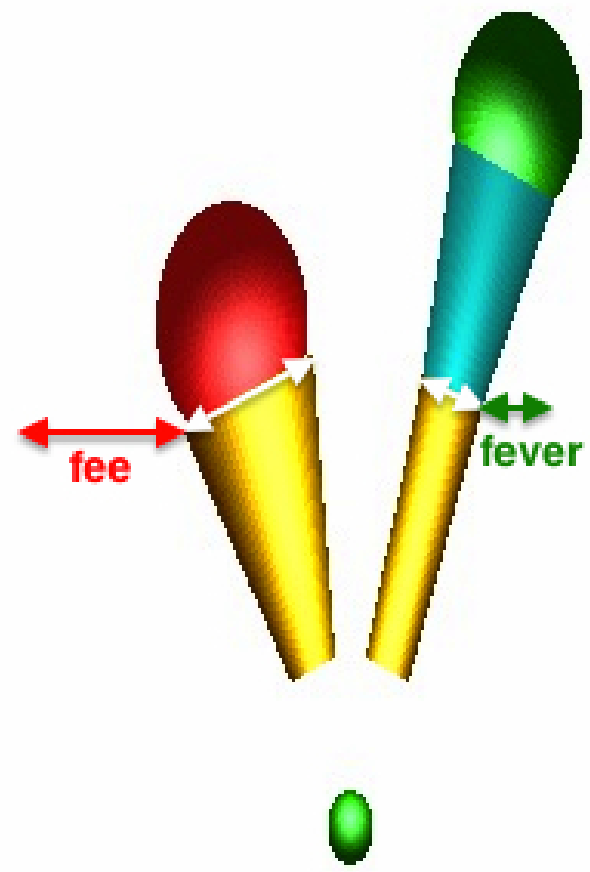

Figure 3.21: Annotated Oronym Parse tree generated for the phrase "fever pitch" 


\section{Sunburst diagram generation}

To generate these sunburst diagrams, we modified our existing $\mathrm{C}++$ program to output data in the Protovis.js format, which is seen in Figure 3.22 .

The labels before the colons are displayed on the diagram in their respective segment, as seen in Figure 3.23.

Some minor adjustments were made to the $\mathrm{C}++$ output. The Protovis document format does not allow for non-alphanumeric characters to appear in labels, so words like "ice-cold" or "it's" caused errors. We found it necessary to remove all non-alphanumeric characters so that the sunbursts would generate successfully.

One of the main benefits of the Protovis data format is that, once the relevant data has been formatted correctly, many different types of graphs can be trivially generated. For our data format, we can generate both sunburst and icicle graph views. We chose to use sunburst graphs, as results from an informal user poll indicated a preference for sunbursts over icicles.

\section{Reading a sunburst diagram}

To read a sunburst diagram, start at the very center, which in our case is labelled "root". Then, pick any one segment from the first ring surrounding the root. The word contained in this segment will be the first word in the oronym phrase.

Next, look at all the outer arc segments that directly touch the first segment picked. Every word in those adjoining arc segments is a valid subsequent word for the oronym phrase starting with the word in the first, inner arc segment. 


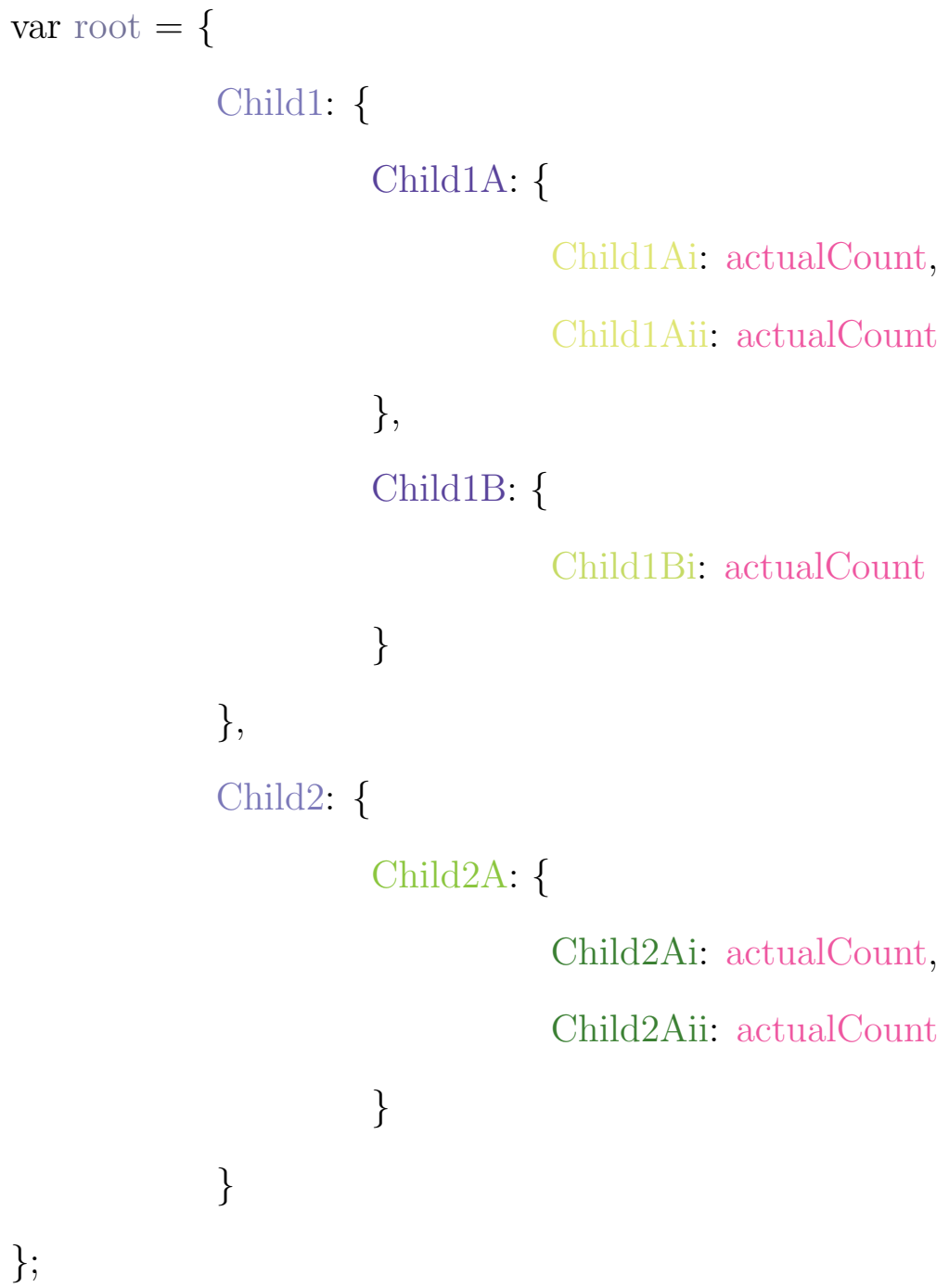

Figure 3.22: Protovis sunburst data format: This example sunburst data file, once the actualCount occurances were replaced with actual values, would generate a sunburst diagram 


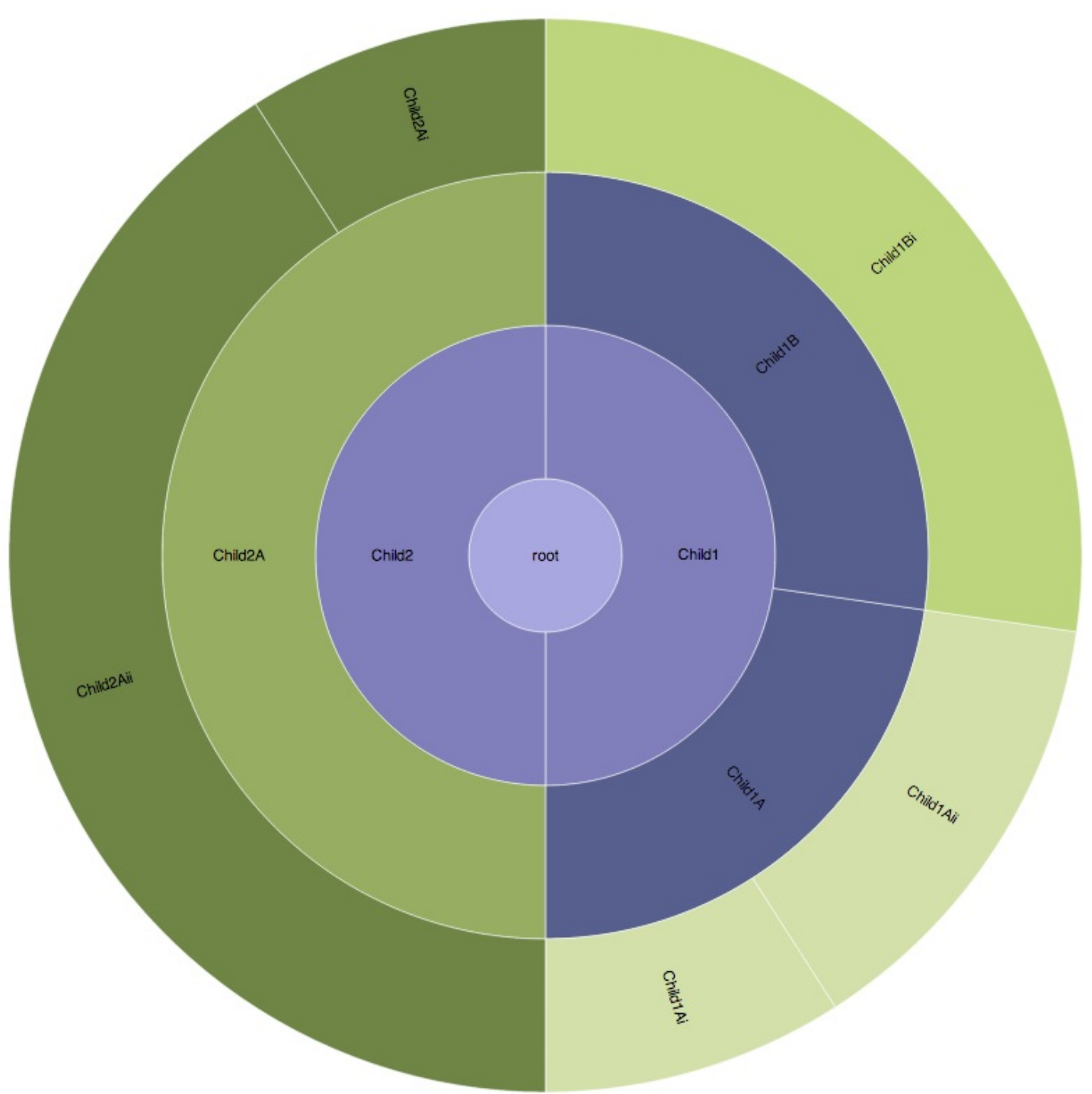

Size: Paths weighted by demo freq metric

Figure 3.23: This example sunburst diagram is what would be generated by the example data file in Figure 3.22 
Continue this process, using one segment per level, until you reach a segment that has no subsequent outer segments. At this point, you will have compiled a full oronym phrase. The size of the final outer segment, relative to size of the rest of the segments in its particular ring, shows you the relative commonness of the phrase whose path ends at that segment.

So, for example, interpreting the sunburst diagram in Figure 3.23 would result in the following faux-oronym phrases: "child1 child1A child1Ai", "child1 child1A child1Aii", "child1 child1B child1Bi", "child2 child2A child2Ai", and finally, "child2 child2A child2Aii".

\section{Example Sunburst Diagrams}

We generated several types of sunburst diagrams, using both artificiallybalanced path weights and the frequency values for each path derived from our UNISYN dictionary. A clearer view of how we used sunburst diagrams can be provided with some concrete examples.

Consider the two sunburst diagrams for the oronyms of the phrase "iced ink", shown in figures 3.24 and 3.25. The phrase "iced ink" has five different oronyms: "iced ink", "ay stink", "aye stink", "eye stink", and "I stink". The five different outer segments (which are easier to see on the equal-weighted sunburst diagram in Figure 3.24) represent the end word of each of those oronyms. The sunburst diagram that uses the frequency metric (shown in Figure 3.25) shows that people are overwhelmingly more likely to hear "I stink" than any other possible oronym.

For a more complicated example, take the sunburst diagrams for oronyms of the phrase "why that's insane". The diagram shown in Figure 3.26 shows the seven possible oronyms that can result from phonetic interpretation of that 


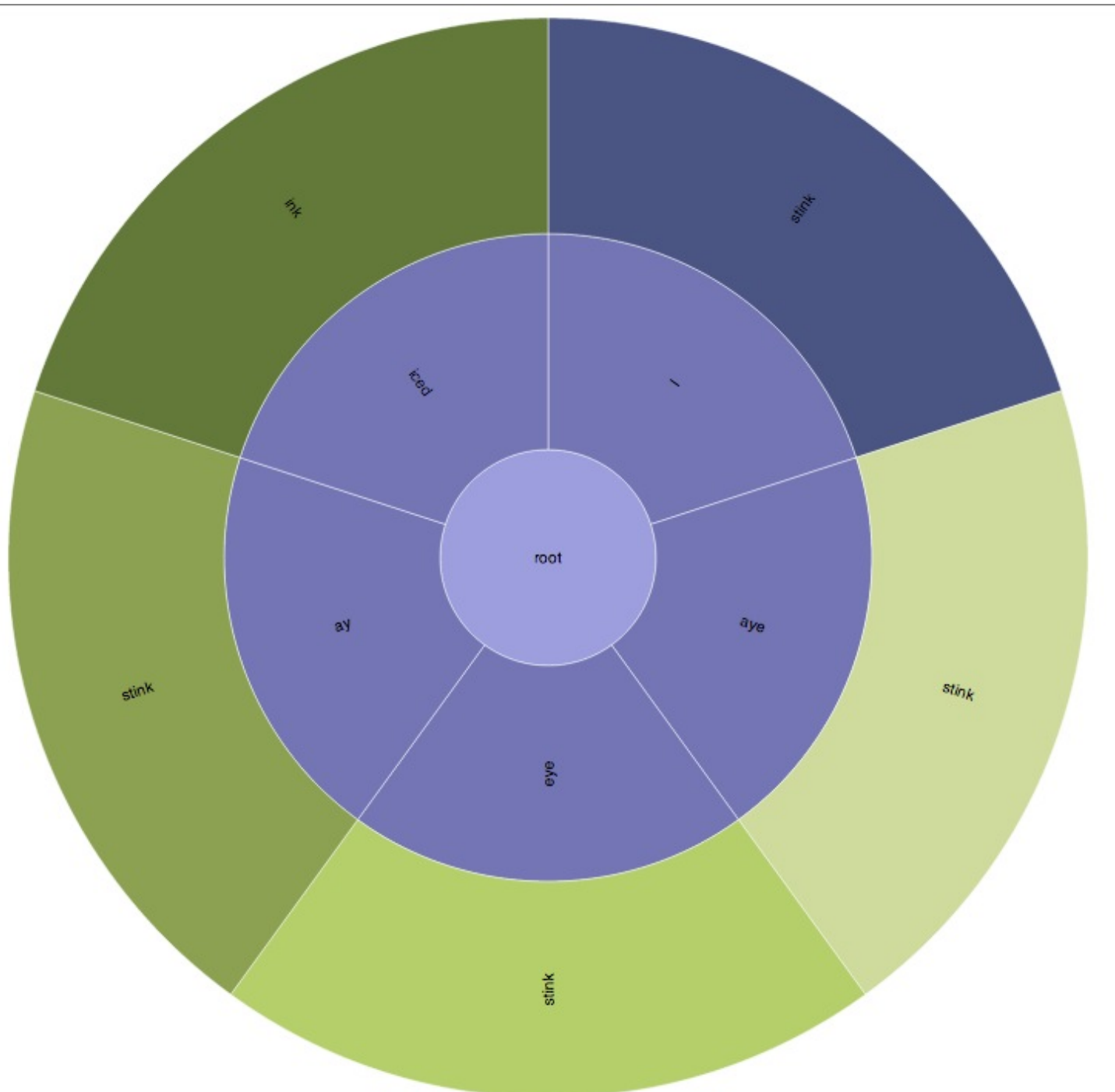

Size: Equal Weight to all paths

Figure 3.24: Equally-Weighted Sunburst Diagram for the oronyms of "iced ink" 


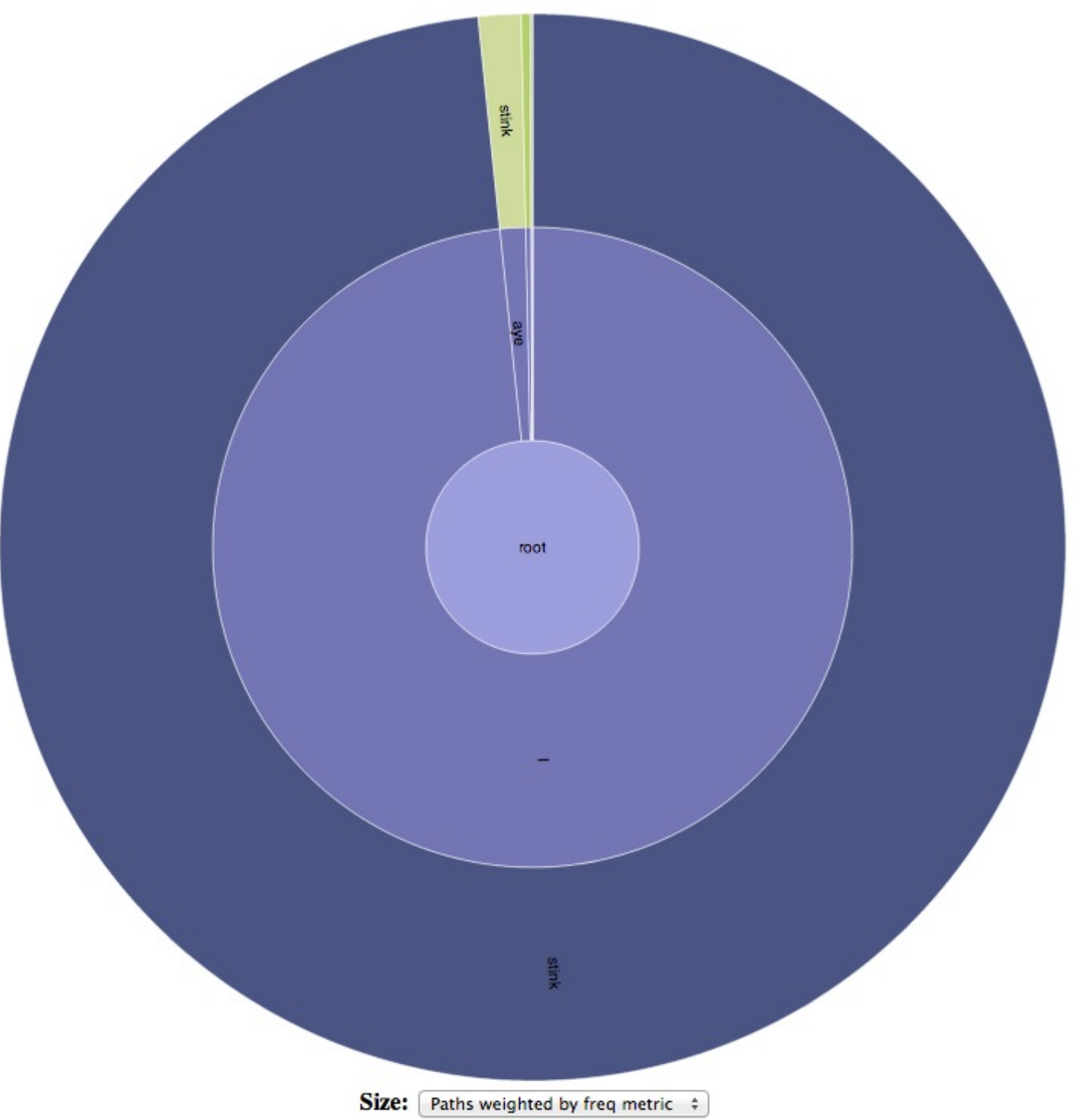

Figure 3.25: Sunburst Diagram for the oronyms of "iced ink" weighted by UNISYN freq metric 
phrase, and even though one phrase is one word shorter than all the rest, the weights of the paths remain equal. The weighted diagram, shown in Figure 3.27, shows the UNISYN-predicted frequency of each phrase's occurance.

Lastly, consider the sunburst diagrams for the phrase we chose to focus on in our user study: "an ice cold hour". As seen in Figure 3.28, the equallyweighted sunburst diagram shows all possible oronym paths. When compared to the sunburst diagram in Figure 3.29 that uses the UNISYN-derived frequency metric, we can see that some paths, such as those that begin with the word "a", are much more likely to be heard than those that begin, for example, with the word "n". 


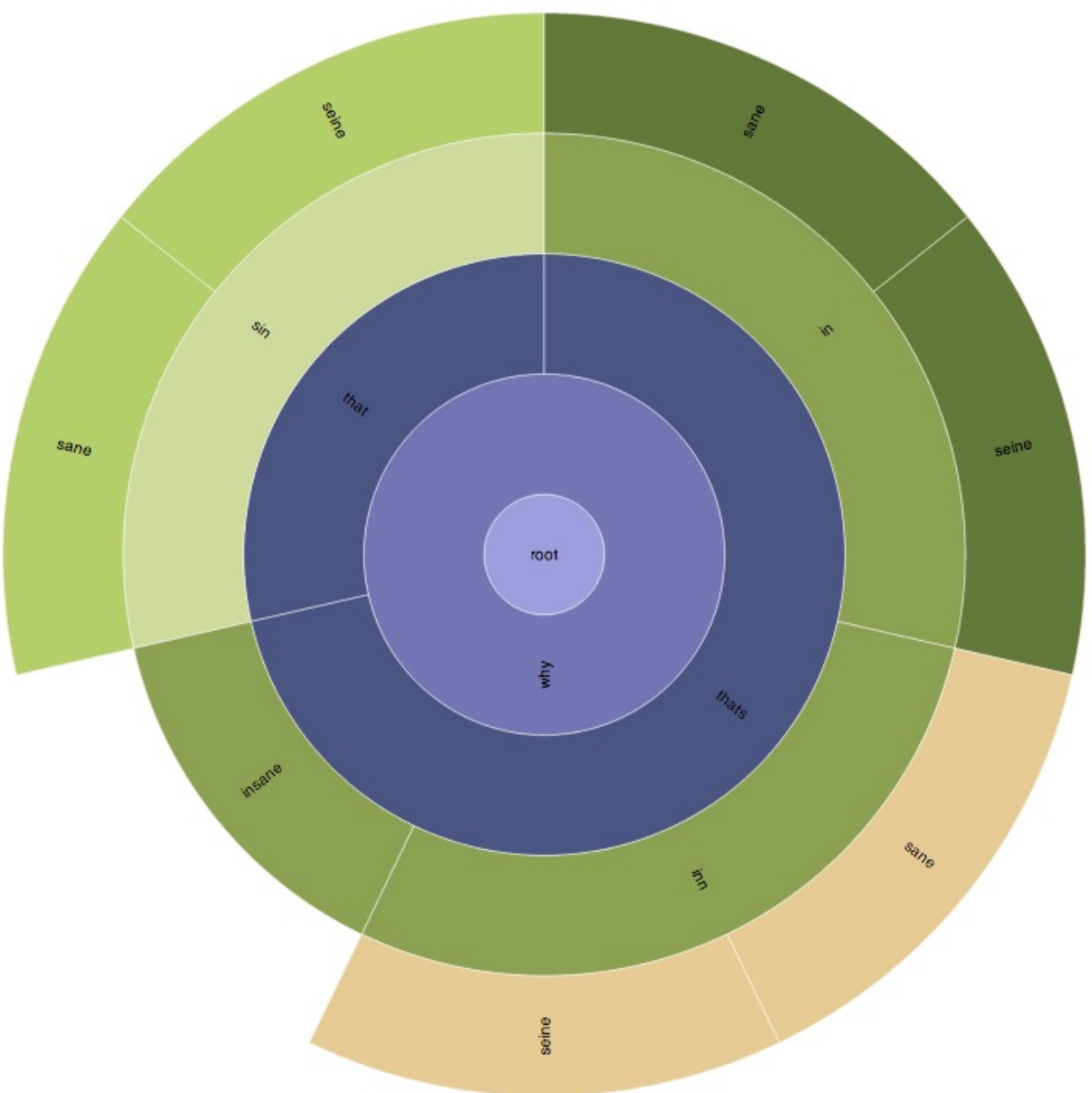

Size: Equal Weight to all paths

Figure 3.26: Equally-Weighted Sunburst Diagram for the oronyms of "why that's insane" 


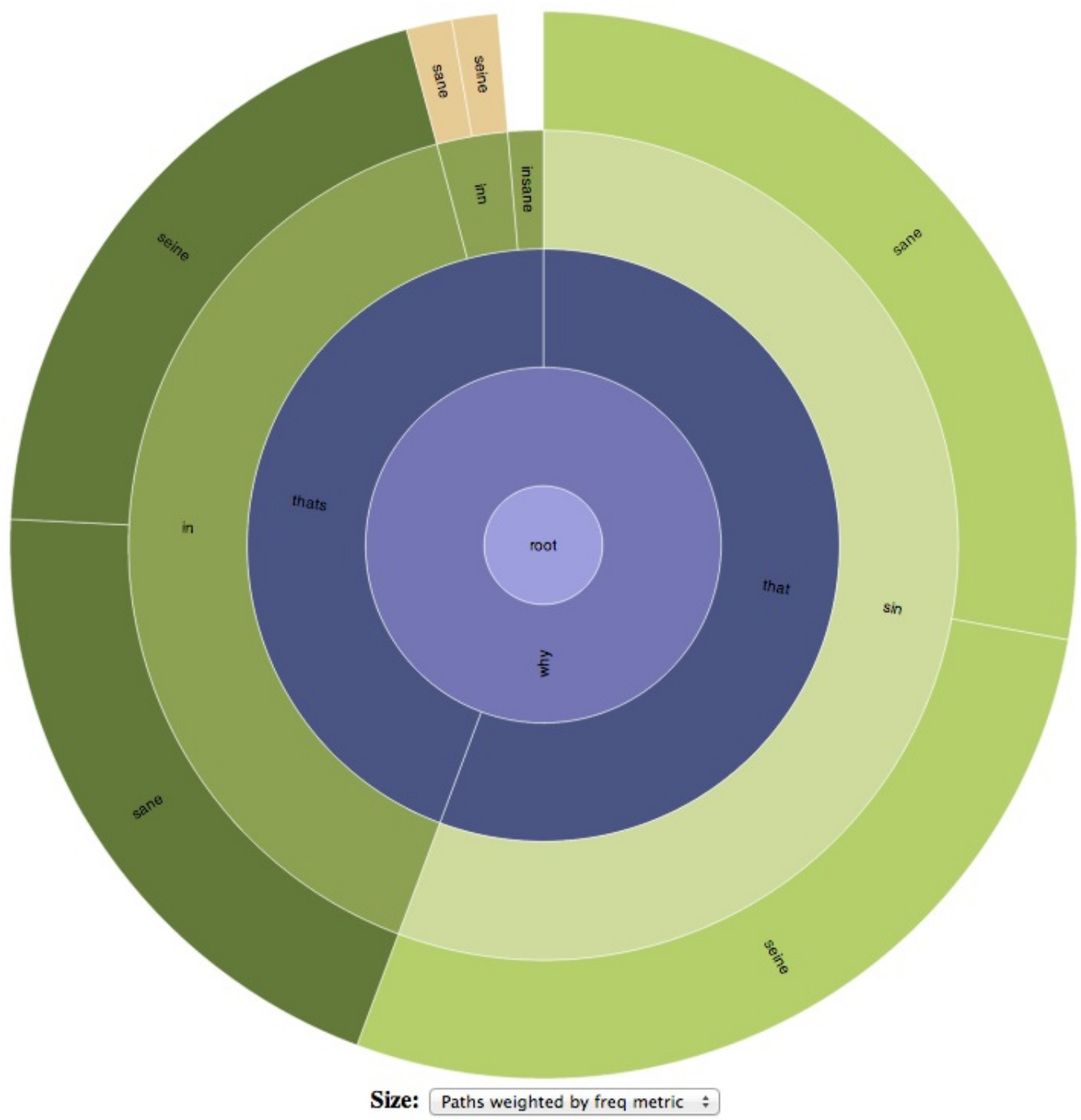

Figure 3.27: Sunburst Diagram for the oronyms of "why that's insane" weighted by UNISYN freq metric 


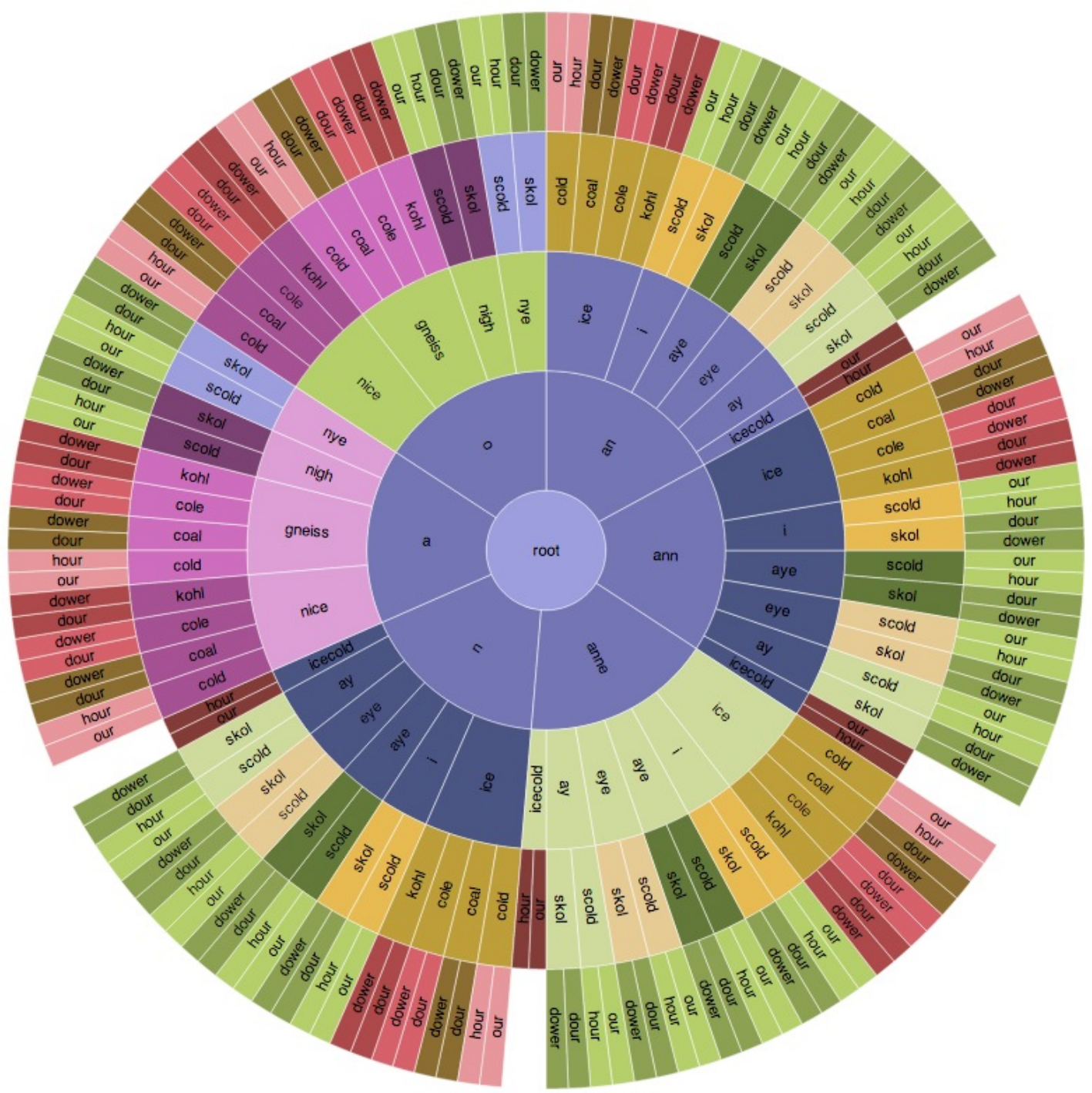

Size: Equal Weight to all paths

Figure 3.28: Equally-Weighted Sunburst Diagram for the oronyms of "an ice cold hour" 


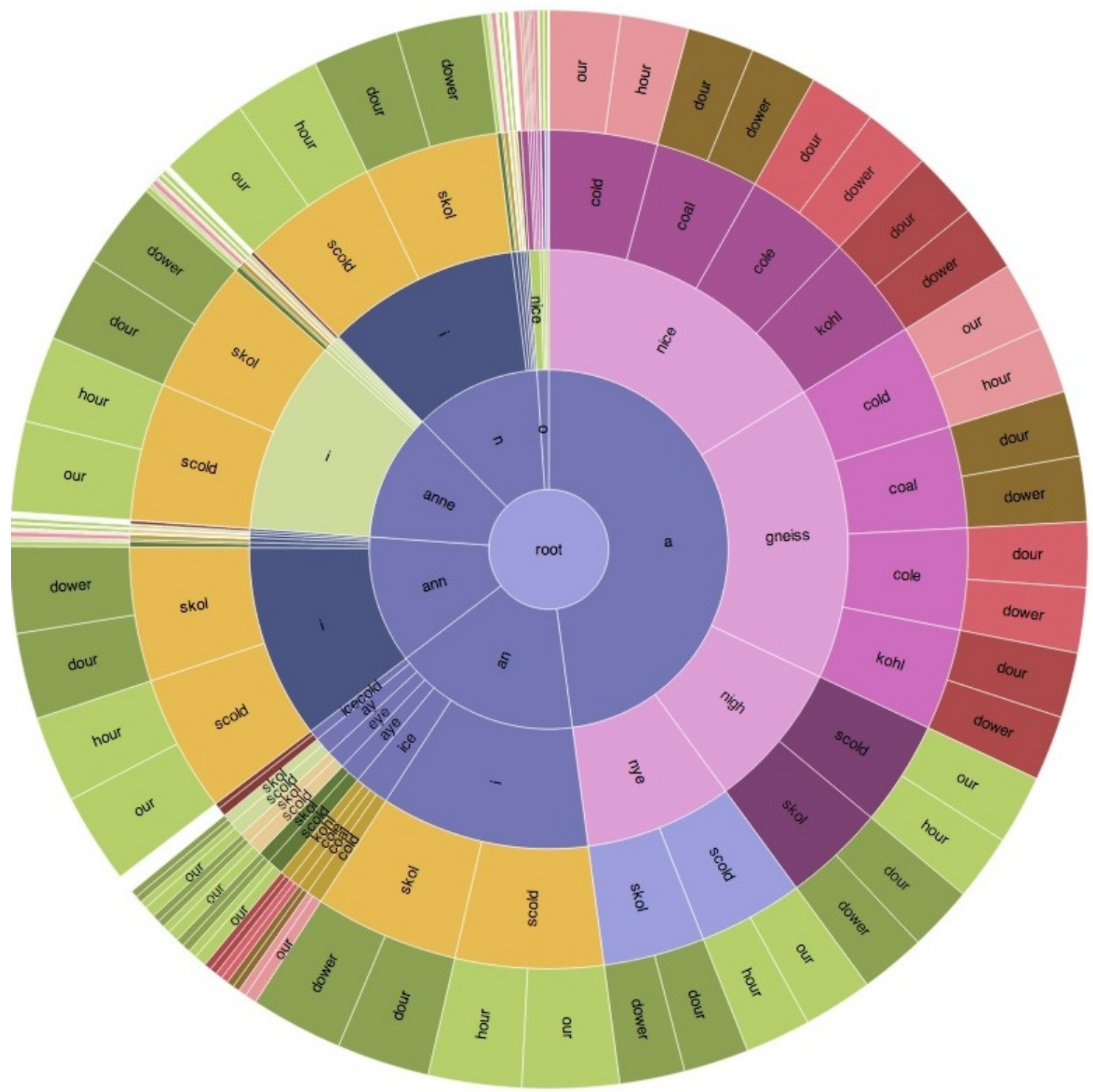

Size: Paths weighted by UNISYN freq metric

Figure 3.29: Sunburst Diagram for the oronyms of "an ice cold hour" weighted by UNISYN freq metric 


\section{Chapter 4}

\section{User Study}

\subsection{Purpose}

We created a multi-phase user study to examine the correctness of our method of word frequency metric generation, which uses the UNISYN dictionary.

We chose to develop a user study to see if our calculated frequency values were a good match for real-world expectations. In the event that our calculated frequency values of the oronyms for a root phrase mirrored the observed frequency of various user transcriptions of that same root phrase, then we could conclude that our source frequency dictionary accurately reflected reality. In the event that the expected and observed frequencies diverged, we could conclude that our source for frequency values was flawed.

During the course of our study, we found that using per-occurance frequency values, like those found in the UNISYN dictionary, when computing our overall-

phrase-frequency metric caused the end result to be thrown off by excessively common words, such as "the", "is", and "a". These super-common words had 
such high per-occurance tallies that they overpowered any effect that any regular word had on a frequency metric. We found that phrases that were relatively rarely observed had high expected frequency values. As a result, we do not recommend using the UNISYN dictionary for word frequency purposes.

\subsection{Structure}

First, we generated oronyms for the phrases "a nice cold hour" and "fourth rye to". Using the selection criteria outlined in section 4.4.1, we narrowed down our recording options to 48 out of the 290 oronyms generated for "a nice cold hour", and 10 out of the 39 oronyms generated for "fourth rye to".

In the first phase, we had a dozen people record over 72 recordings of 58 different phrases. This phase served two purposes: one, to see if our phonemic transcriptions were valid, and two, to gather recordings for the second phase.

In the second phase, we took 15 recordings of oronyms from phase one, and gathered approximately 74 transcriptions for each recording, resulting in a total of 953 transcriptions. These transcriptions were provided by 208 unique users ( 127 from the United States). We then compared the observed frequency of transcriptions of the recorded oronym phrases to the calculated frequency metric for oronyms of the original root phrase.

\subsection{User Sampling Population}

We drew our test subjects from a pool of Amazon Mechanical Turk workers

(hired for $\$ 0.02$ to $\$ 0.10$ per task) and, for part of phase 1 , volunteers from 
Reddit.com [4] [5].

Amazon Mechanical Turk is an online crowdsourcing service where requesters can hire workers to complete Human Intelligence Tasks, or HITs. The efficacy of using Mechanical Turk for user studies has been widely studied in academia, and specifically proven in the linguistic community [30].

Due to the online nature of Mechanical Turk, we we able to gather an international pool of volunteers. As you can see in figure 4.1, the bulk of our responses came from the United States and India.

\subsection{Methodology}

\subsubsection{First Phase: Recitation}

In this phase of the user study, we used a combination of a dozen Mechanical Turk workers (hired for $\$ 0.10$ per task) to record 72 different phrases. These phrases were oronyms of one of two phrases: phrase A, "a nice cold hour" or phrase B, "fourth rye to".

Given that "a nice cold hour" has 290 oronyms, and "fourth rye to" has 39 , it was necessary to narrow down the number of oronyms recorded in phase one of our user study.

To select which oronyms we would submit to Mechanical Turk for workers to record, we decided on the following selection criteria:

- We discarded oronyms that included words with frequency values of less than 30. Asking a general audience to pronounce uncommon words would likely result in a high rate of unusable recordings. In addition, including 
uncommon words would not actually test our algorithm-it would test the vocabulary of the user study subjects, which is outside the scope of this project.

- We discarded all oronym phrases that contained words that capitalize to proper nouns, if that capitalization led to alternative pronunciations. For example, "nice" maps to the phonetic sequence n aI s, but "Nice" maps to the phonetic sequence $\mathrm{n}$ i s (as in "niece").

- We discarded oronym phrases that included implicit punctuation. For example, the phrase "Anne I scold our", has an implicit comma between Anne and I. We did this to avoid halting or "dramatic" recording of phrases.

- We discarded oronyms with any words whose pronunciation is position specific. For example, the word " 'n'" is pronounced @ $\mathrm{n}$ when found in "Rock 'n' Roll, but when on its own, is pronounced $\mathrm{E} n$, which does not map back to the original root phrase "an ice cold hour". We also removed some phrases involving the word " o" ". When pronounced @, as it is in "top o' the morning", it maps back to the original root phrase, but when found outside of that phrase, as in a last name like O'Donnell, it is pronounced oU , and does not map back.

- We only chose oronyms for which all pronunciations of the child oronym phrases were also found in the pronunciations of the root oronym phrase. For example, a root phrase that begins with "a" would have an child oronym phrase that begins with "et", using French pronunciation which drops the trailing t sound. However, "et" can also be pronounced with the t, using an American accent, as in the phrase "et al". Since our root phrase does not include that $t$ sound, any child oronym phrases that begin with 
"et" have at least one pronunciation that does not match the root phrase's pronunciation. So, we discarded all child oronyms that begin with "et".

At the end of this process, we were left with 48 out of the 290 oronyms generated for "a nice cold hour", and 10 out of the 39 oronyms generated for "fourth rye to".

To keep track of the chosen phrases, we assigned each phrase a phraseID, built off of the phrase letter, phrase length, and phrase text. We gave Mechanical Turk workers three minutes to record each phrase and email it to us with the phrase identifier in the subject of the email. The number of recordings per phrase, along with their identifiers, can be seen in table D.1.

We then interpreted the phonetics of each of the recordings in SAMPA by ear. In a stunning example of a use case for our project, we discovered that we had unintentionally included some phrases for recording that were not deterministically phonetically parsable, meaning that our oronyms had multiple pronunciations, not all of which mapped back to the original phrase. While we had caught and removed the phrases that began with "et", our algorithm mapped the orthographic word "a" to the phoneme A (as in "a capella" or "father". That A phoneme can be combined with the subsequent $\mathrm{n}$ phoneme from the word "nice" to create the SAMPA sequence A $\mathrm{n}$, which corresponds with the orthographic word "on". Since that does not map back to our original root phrase, we were forced to discard all transcriptions that ended up with that pronunciation. That being said, the remaining pronuciations fit with our model, and we found no other unexpected phonetic anomalies when comparing our recordings to the expected SAMPA pronunications of each phrase. 


\subsubsection{Recording Sample Pool}

We had originally intended to use every phase one recording in phase two, but had to discard all but 15 of the recordings for various reasons, the most common being that the recording was too loud and we wanted to spare our users ears. Occasionally, the person recording left excessive amounts of space between words that overly segmented the phrase, interrupting the natural flow of the phonetic sequence and rendering it unusable for our purposes. The recordings for the "fourth rye to" oronyms were all unusable for phase two, because our users tended to insert exclamation points any time they said "ooh" or "too", overloading their microphones or over-segmenting the phrase[24].

All 15 recordings we used were oronyms for the phrase "a nice cold hour", and were recorded by one man from the midwest, whose accent which made him the best approximation we could get for a General American accent. All other suitable recordings gathered in phase one did not have appropriate accents, and were summarily discarded from further data collection.

\subsubsection{Second Wave: Transcription}

As previously stated in section 4.2, we hired 208 unique Mechanical Turk workers to transcribe our oronym recordings for $\$ 0.02$ to $\$ 0.03$ per transcription.

Each of the 15 recordings was transcribed approximately 74 times, resulting in a total of 953 transcriptions. These transcriptions were provided by 208 unique users ( 127 from the United States). In addition to transcribing the recording, in each task the worker was asked what country they were from. We did this to help differentiate native American English speakers from non-native speakers, in order to draw interesting conclusions about the differences in native- and non-native 


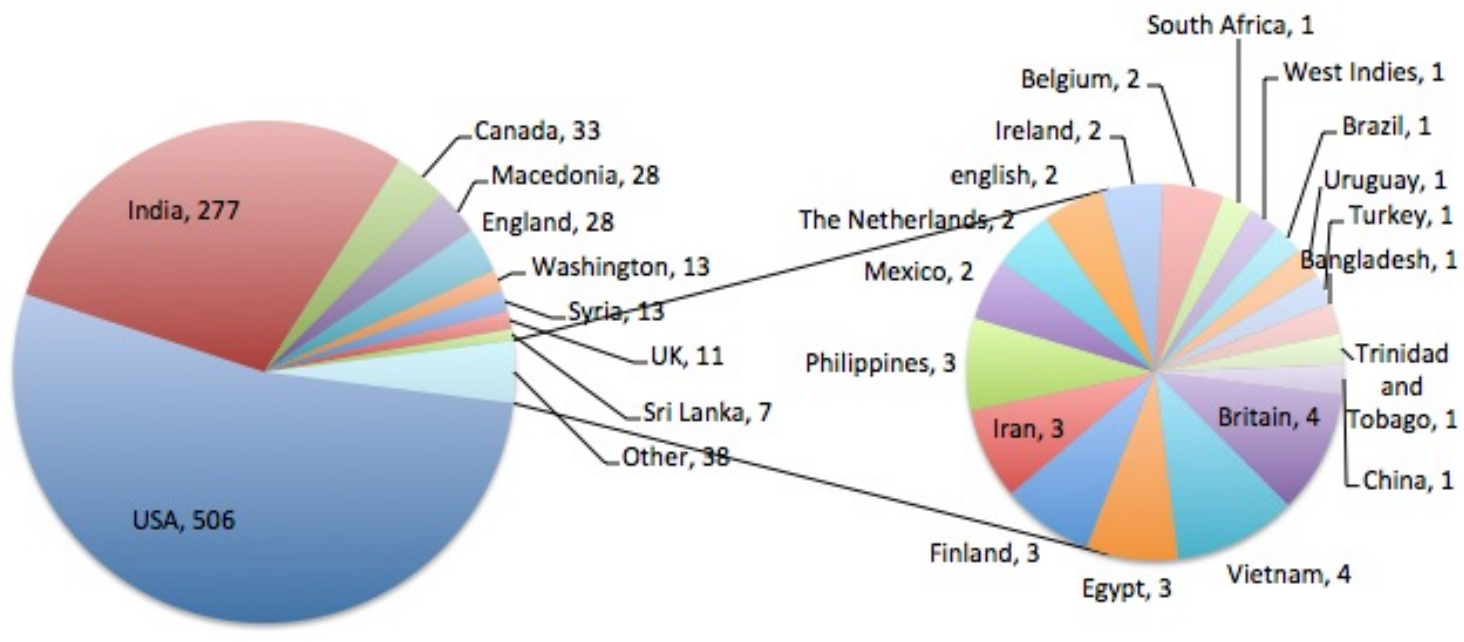

Figure 4.1: Our user study primarily polled people from the United States and India, as can be seen by the number of responses originating from each country.

speaker phrase parsing. 


\section{Chapter 5}

\section{Results}

\subsection{Phase One Results}

As previously discussed in Section 4.2, In this phase, we recorded a dozen users reciting any of the 48 oronyms of the phrase "an ice cold hour", or any of the 10 oronyms for the phrase "fourth rye to".

Out of 72 recordings, only the recordings of the oronyms of "fourth rye to" were found to diverge from our expected phonetic patterns, likely due to poor microphone quality not being able to pick up the aspirated ' $f$ ' sound at the beginning of the phrase[24]. All other oronyms were found to be within reasonable tolerance levels, with 15 recordings from one particular speaker found to be a close enough match to the General American accent to use his recordings in phase two. 


\subsection{Phase Two Results}

As previously outlined in Section 4.2, we gathered 953 transcriptions for our 15 recorded phrases, with each recording garnering approximately 74 transcriptions.

Worldwide, the top four most-frequently transcribed phrases made up for $70 \%$ of total transcriptions. The top transcribed phrase worldwide was "an ice cold hour", with 352 transcriptions, followed by "a nice cold hour", with 217 transcriptions. Following that, "a nice gold hour" had 63 transcriptions, and "in ice cold hour" had 38 transcriptions. The breakdown of these top four can be seen in figure 5.1 and table 5.1 .

All of the worldwide top transcribed phrases were predicted by our oronymgenerator, except for "a nice gold hour". This is a known limitation of our project, though, because we chose to focus on exact phonetic matches. The cold/gold mishearing is a product of phoneme voiced/voiceless pair swapping, which we cover in-depth in Section 5.4.2. It is outside the current scope of our project.

\subsubsection{Transcribed oronyms' observed frequency vs. ex- pected frequency}

Though the most commonly transcribed phrases were predicted by our method of oronym generation, figure 5.3 shows an unexpected distribution of the number of times each phrase was transcribed versus the frequency metric that we calculated. We hypothesized that a simple summation of the UNISYN-provided word frequencies for each word in a phrase would produce a meaningful indicator of a 


\section{Worldwide Count}

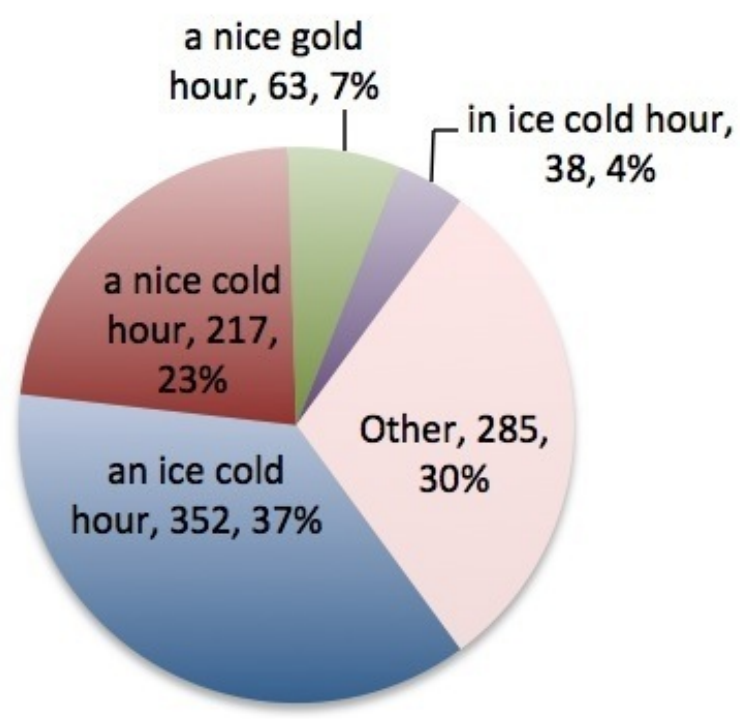

Figure 5.1: Our top two transcriptions were "a nice cold hour" and "an ice cold hour"

\begin{tabular}{|l|l|l|}
\hline predicted freq & phrase transcribed & total answers \\
\hline 931028 & an ice cold hour & 352 \\
\hline 7851662 & a nice cold hour & 217 \\
\hline 0 & a nice gold hour & 63 \\
\hline 5503158 & in ice cold hour & 38 \\
\hline 0 & an ice gold hour & 18 \\
\hline 859307 & an eye scold hour & 13 \\
\hline
\end{tabular}

Table 5.1: In this table, we list all oronyms that were transcribed more than five times. Out of this list, all but the two containing the word "gold" were predicted by our oronym algorithm. However, we expected that any voiced/voiceless phoneme substitutions, like "cold" / "gold" would be missed by our algorithm. 
phrase's likelyhood to be heard.

Unfortunately, that proved not to be the case. In figure 5.2, we see the sunburst diagram for the expected distribution of transcribed phrases based upon the UNISYN frequency metric that we calculated. In figure 5.3, we see the sunburst diagram for the transcriptions we observed, with a special slice representing all the transcriptions we did not predict. The unpredicted slice is nearly as large as the slice for "an ice cold hour", which was observed the most often out of all expected transcriptions.

\subsubsection{Statistical measurement of expected versus observed transcription frequency}

A statistical analysis of the observed dataset versus the expected dataset, using a one-proportion z test, further proves that the calculated UNISYN frequency values were not a good predictor of observed transcription. Using the top two observed transcriptions as our sample population, we take a look at the phrases "a nice cold hour" and "an ice cold hour" . The phrase "a nice cold hour" has a calculated UNISYN freq metric of 7851662 , and had 125 actual transcriptions observed among people living in the United States. The phrase "an ice cold hour" has a calculated UNISYN freq metric of 931028, and had 191 actual transcriptions observed among people living in the United States. Therefore, the expected population is 8782690 , and the observed population is 316 .

Given those populations, the expected population proportion for "a nice cold hour" would be $7851662 \div 8782690$, or 0.8934 .

In our user study, we found that 125 people transcribed "a nice cold hour" , and 191 people transcribed "an ice cold hour", for a ratio of 0.65 to 1, where "a 


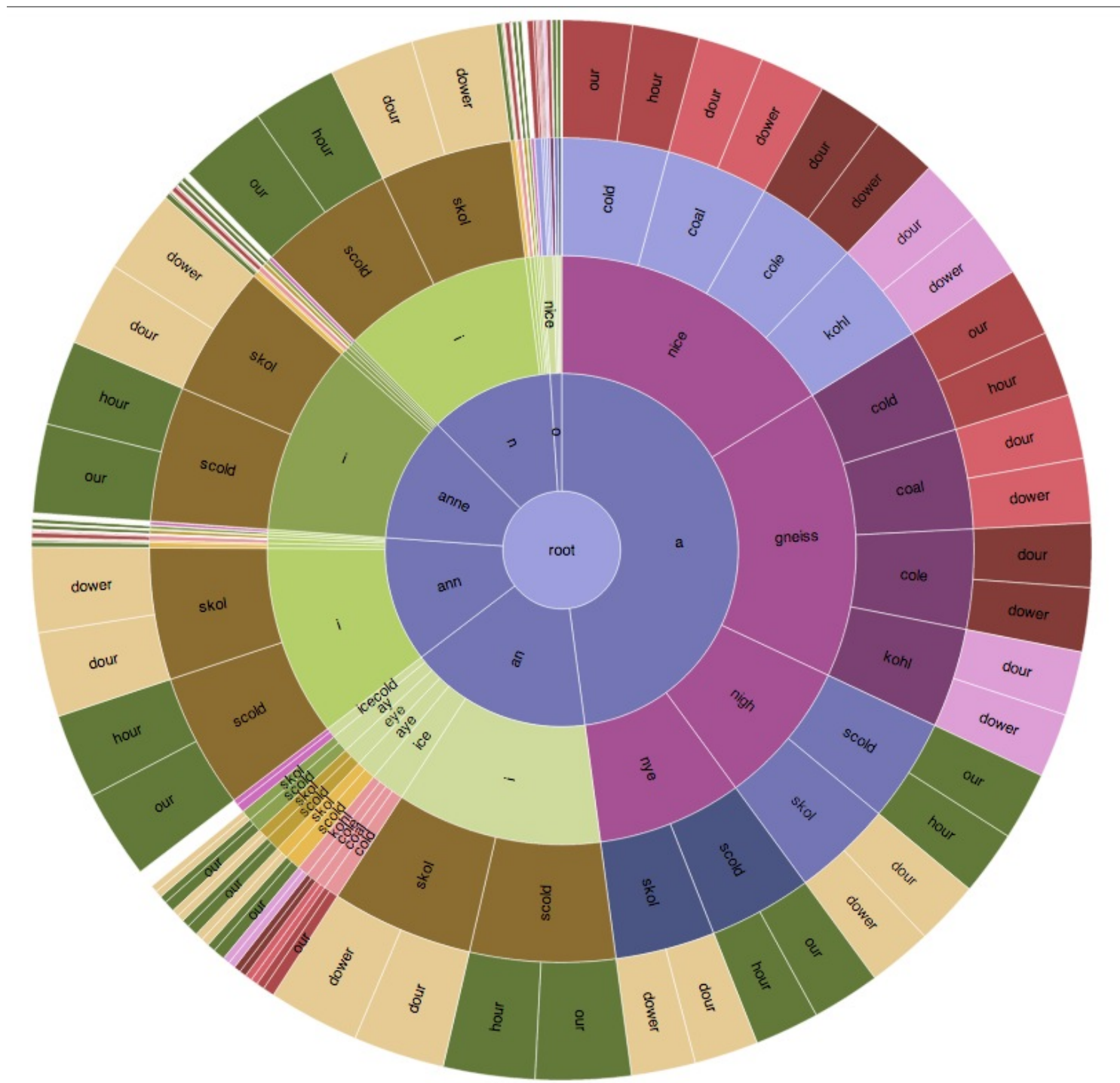

Size: Paths weighted by UNISYN freq metric

Figure 5.2: Sunburst Chart for A Nice Cold Hour using UNISYN metrics for comparison to observed frequency sunburst 


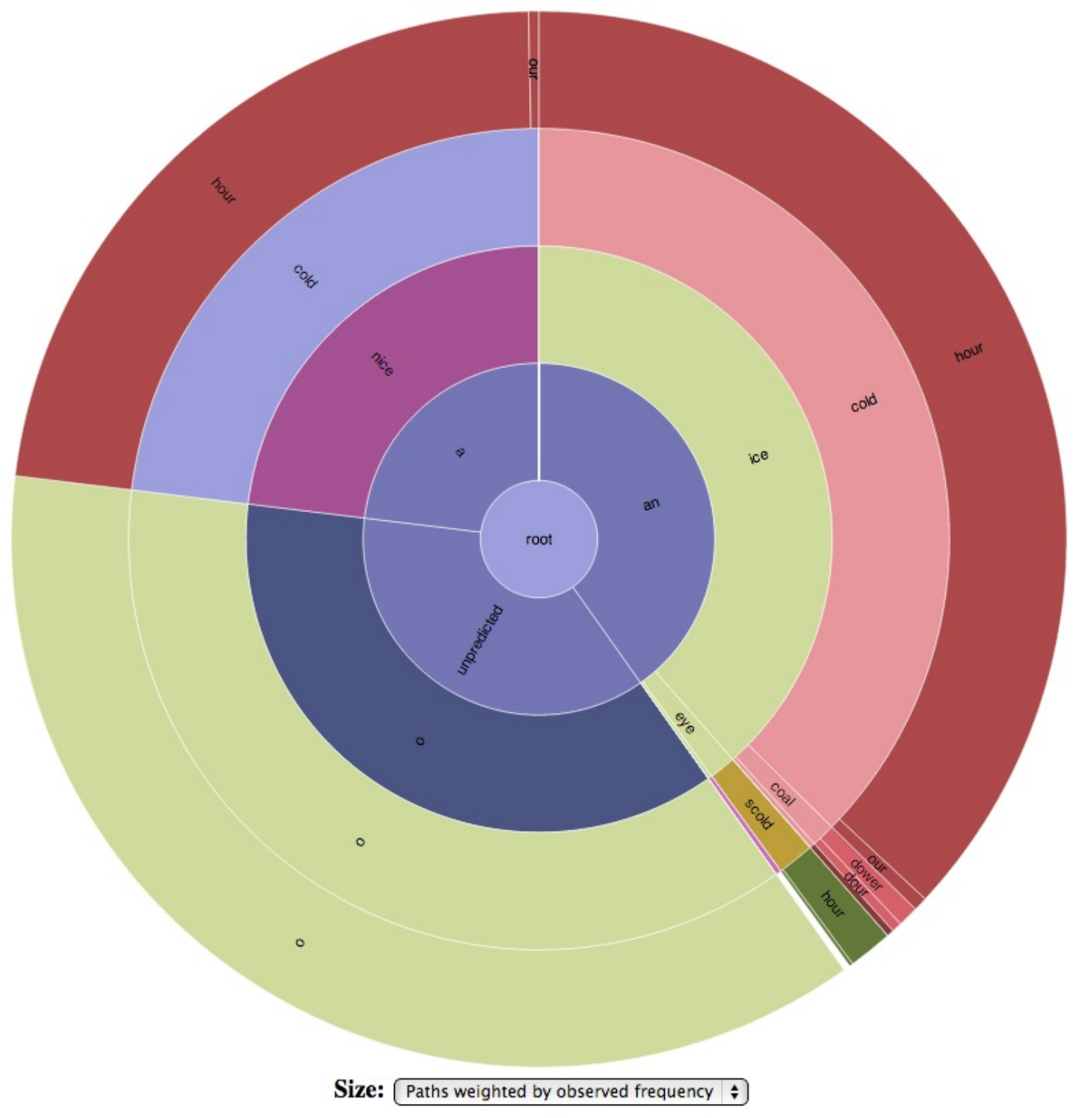

Figure 5.3: Sunburst Chart for A Nice Cold Hour using observed frequencies 
nice cold hour" accounts for 39.56\% ( $\mathrm{p}=0.3956)$ of the combined count.

Given the observed population proportion of 0.3956 and the expected population proportion 0.8934 , we did a one-proportion z test with an $\alpha$ of 0.01 . The $\mathrm{z}$ value returned was 18.0971 , meaning that the observed population proportion was 18.0971 standard deviations away from the expected population proportion. When we used this $\mathrm{z}$ value to compute a $\mathrm{p}$ value, we got a value so low that we were unable find a calculator with enough decimal places to show it without rounding it to zero.

In short, the per-occurance frequency metric predictions derived from UNISYN do not even remotely match the observed data.

\subsubsection{Observations on Transcription Count per Recording for each transcribed phrase}

The Transcription Count by Recording graphs in figures 5.4 through 5.10 show how many occurances of a certain transcription were produced from each recording. Each graph represents one transcription, and has bars for each recording, where each bar shows how many times the transcription was observed for that particular recording. The graph also compares the observed incidences of those transcriptions with the expected UNISYN frequency metric. The $\mathrm{X}$ axis lists the transcribed phrase. The right $\mathrm{Y}$ axis corresponds to the smaller, multicolored bars. Each bar represents the number of times that a transcribed phrase was observed for that particular recording. The left $\mathrm{X}$ axis corresponds to the large blue bar behind the smaller bars. The blue bar represents the calculated UNISYN frequency metric for the transcription.

When you compare the bars from the two y axes, some interesting patterns 
appear.

\section{An ice cold hour}

When looking at figure 5.4, we notice that all but 12 out of the 362 transcriptions of "an ice cold hour" come from recordings of phrases that similarly begin with "an". This suggests the existence of some unmeasured value related to pronunciation that makes the theoretically-identical phonetic sequences of "an ice cold hour" and "a nice cold hour" be heard as functionally different. However, note that in figure 5.4, the blue bar representing the UNISYN frequency prediction for the transcribed phrase underpredicts the number of transcriptions from recordings that begin with "an", while doing a fairly good job of predicting transcription incidence for recordings that begin with "a".

\section{A nice cold hour}

In figure 5.5, we see that, while most of the transcriptions of "a nice cold hour" came from recordings of phrases that begin with 'a', a not-inconsiderable number came from the recordings for the phrases "an ice cold our" and "an icecold our". When taken in regards to the conclusions we drew from figure 5.4, we can conclude that, while listeners appear not to be able to hear an 'a' as an 'an', listeners can, under certain circumstances, hear an 'an' as an 'a'. The blue prediction bar shows that the expected incidence over all recordings was much higher than the observed incidence, and only came close to being correct for the recorded phrases "a nice cold hour" and "a nice coal dower". 


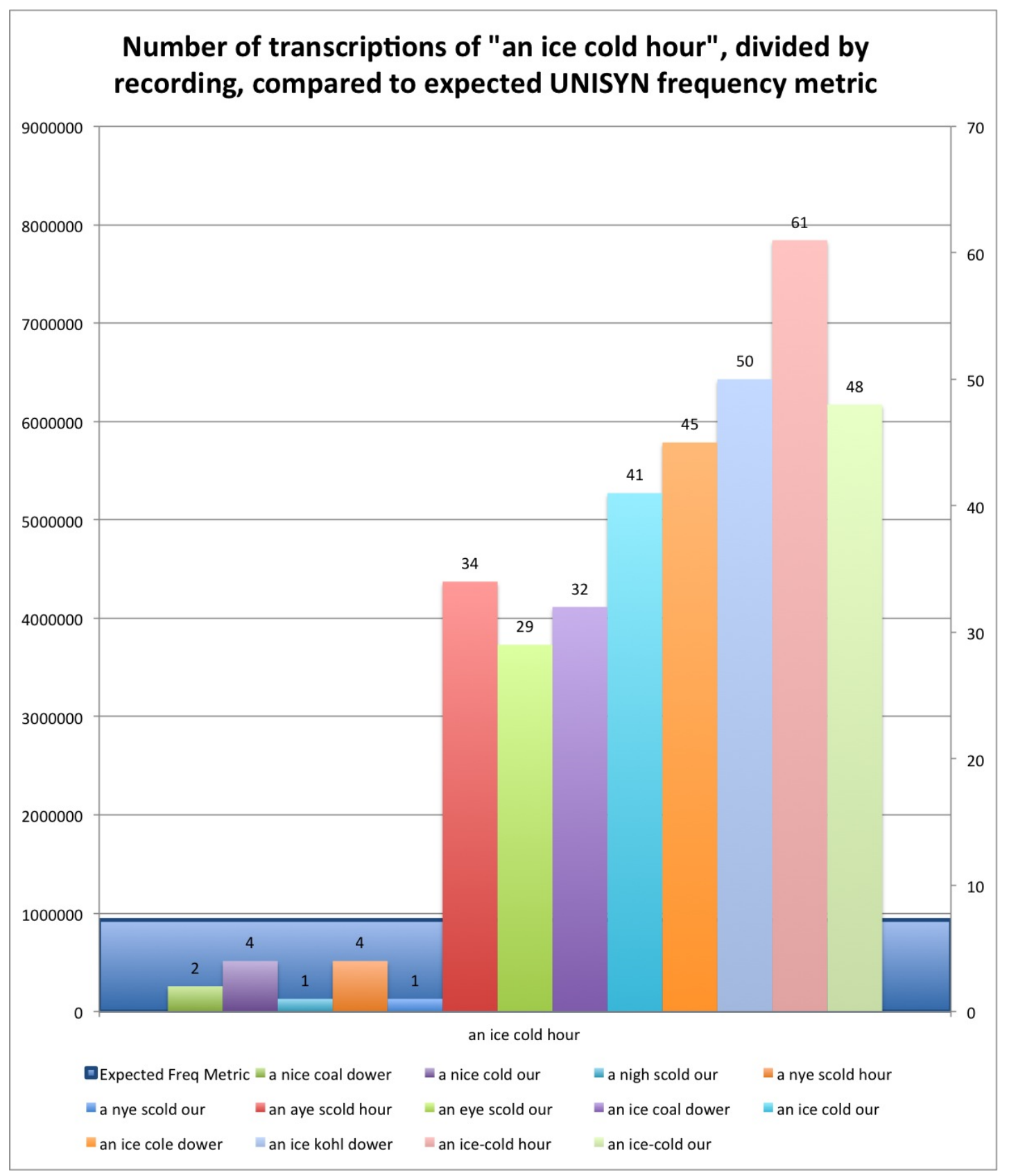

Figure 5.4: This graph represents all transcriptions of the phrase "an ice cold hour", divided into columns based on what recordings were transcribed as "an ice cold hour". The large blue bar in the background shows the predicted frequency metric for the phrase in question. 


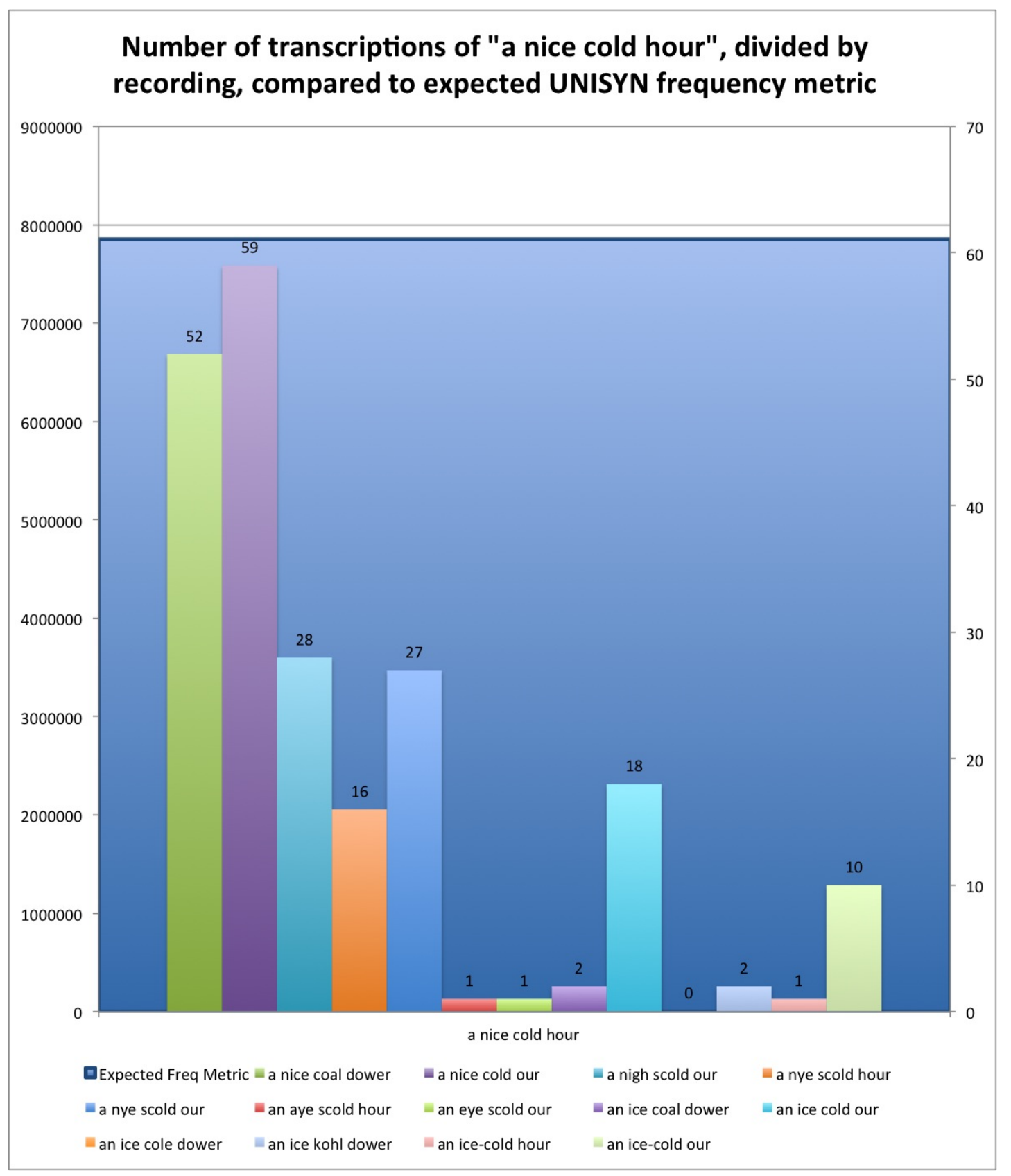

Figure 5.5: This graph represents all transcriptions of the phrase "a nice cold hour", divided into columns based on what recordings were transcribed as "a nice cold hour". The large blue bar in the background shows the predicted frequency metric for the phrase in question. 


\section{In ice cold hour}

The chart in figure 5.6 is for the third most common transcription, "in ice cold hour". Transcriptions of this phrase are fairly evenly distributed among the recordings, though there is a spike of transcriptions for "an ice-cold hour". Additionally, similar to earlier observations, the transcriptions occur a lot less frequently than the UNISYN frequency metric bar suggests they should.

\section{A nice gold hour}

The chart in figure 5.7 for the transcriptions of "a nice gold hour" shows that the source recordings of those transcriptions are very specific: only the recordings of "a nye scold our", "a nye scold hour", and "a nigh scold our" produce the ' $\mathrm{g}$ '/ 'c' substitution. In fact, all of our transcriptions that involved the word "gold" arose from these recordings. This suggests a relationship between the phonemes $\mathrm{s} k$ and $\mathrm{g}$, and warrants further investigation, as suggested later in Section 5.4.2. As shown by the lack of a background blue bar in this diagram, this transcription was not predicted by our oronym generation algorithm, due to the aforementioned phoneme swapping.

\section{An ice cold dower}

Figure 5.8 shows the transcriptions for the phrase "an ice cold dower". This phrase, with its neighboring $d$ sounds in "cold dower", features repeated-phoneme auto-deletion or auto-insertion. Repeated-phoneme auto-deletion or -insertion occurs when two identical and adjacent phonemes are blurred into one sound. This can result in the listener putting two phonemes where only one exists (as in this case), or putting one phoneme where two exist. This phenomenon, while 


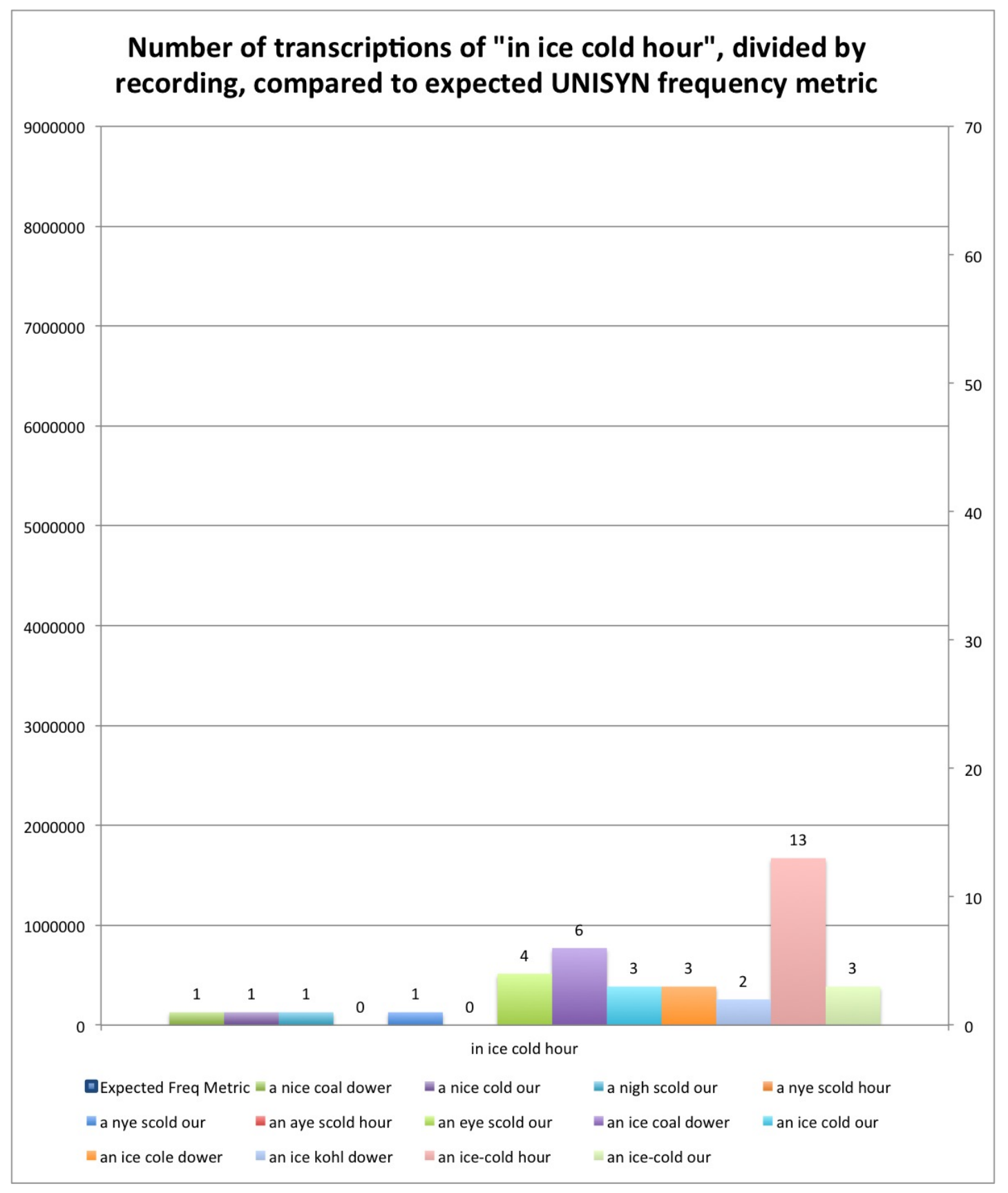

Figure 5.6: This graph represents all transcriptions of the phrase "in ice cold hour", divided into columns based on what recordings were transcribed as "in ice cold hour". The large blue bar in the background shows the predicted frequency metric for the phrase in question. 


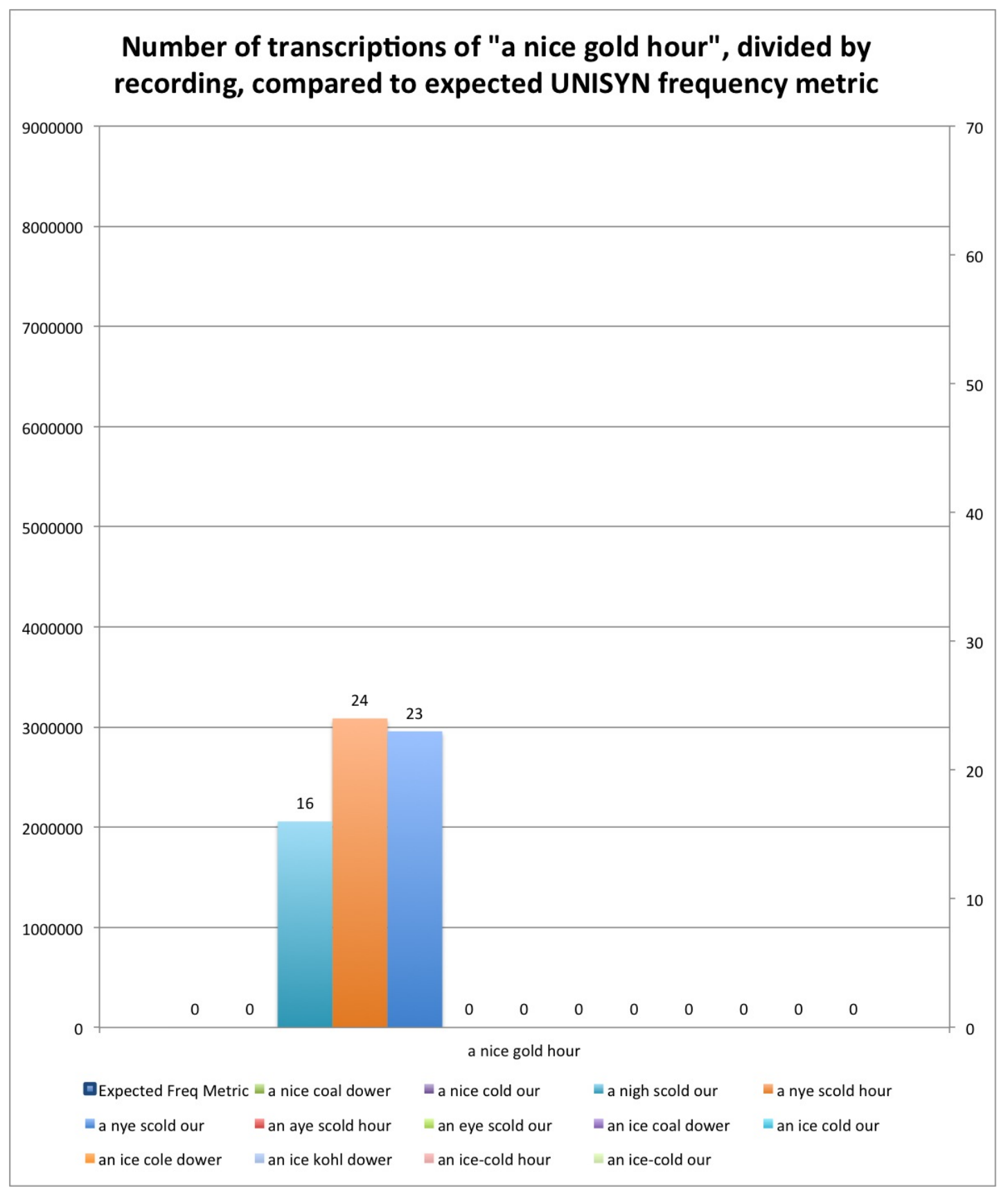

Figure 5.7: This graph represents all transcriptions of the phrase "a nice gold hour", divided into columns based on what recordings were transcribed as "a nice gold hour". The large blue bar in the background shows the predicted frequency metric for the phrase in question. 
known to us, is outside the scope of the current project, and is suggested for future work. Due to this limit in scope, this transcription was not predicted by our oronym generation algorithm, as shown by the lack of a background blue frequency prediction bar in figure 5.8.

\section{An eye scold hour}

The chart for "an eye scold hour", shown in figure 5.9, is primarily interesting in that it appears more or less deterministically interpretable. The only recordings that resulted in this phrase were those for "an eye scold hour" or "an aye scold hour". We hypothesize that this has something to do with word emphases, and suggest investigating this for future work. Interestingly enough, the predicted frequency value was fairly close to the actual occurance ratio for that phrase.

\section{An ice coal dower}

The chart for "an ice coal dower" in figure 5.10 is notable in that all its transcriptions came from recordings that began in "an" and ended in "dower", like the phrase itself does. As in 5.9, we suggest that the near-deterministic interpretation has something to do with emphases, and suggest investigating this for future work. The UNISYN frequency metric once again predicted higher expected incidences than were actually observed for this transcription.

\subsubsection{Transcription Breakdown By Country}

When comparing transcriptions from countries where English is the dominant language (as shown in figure 5.11) to those from countries where it is not (as shown in figure 5.12), we can make some interesting observations. 


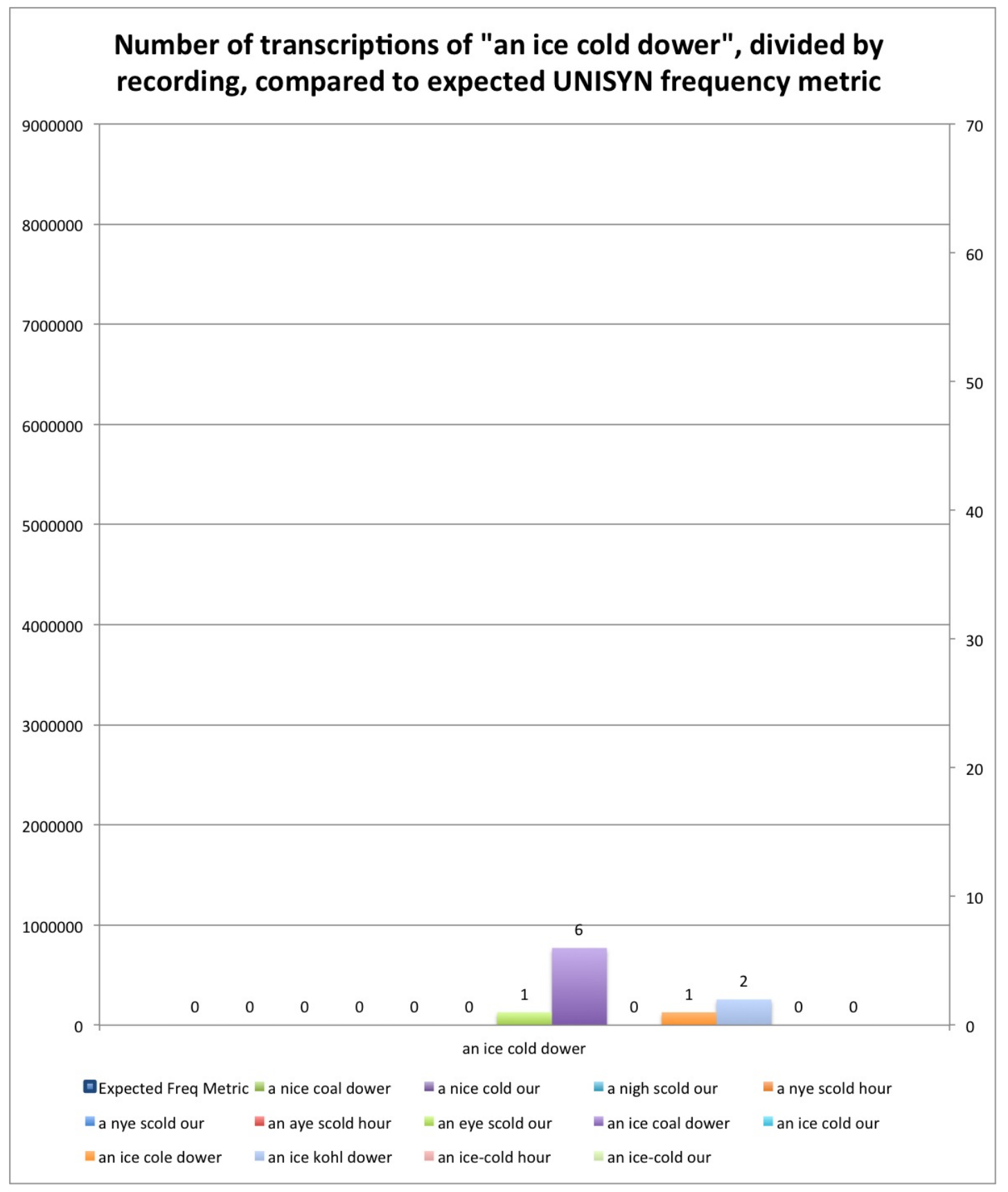

Figure 5.8: This graph represents all transcriptions of the phrase "a nice cold dower", divided into columns based on what recordings were transcribed as "a nice cold dower". The large blue bar in the background shows the predicted frequency metric for the phrase in question. 


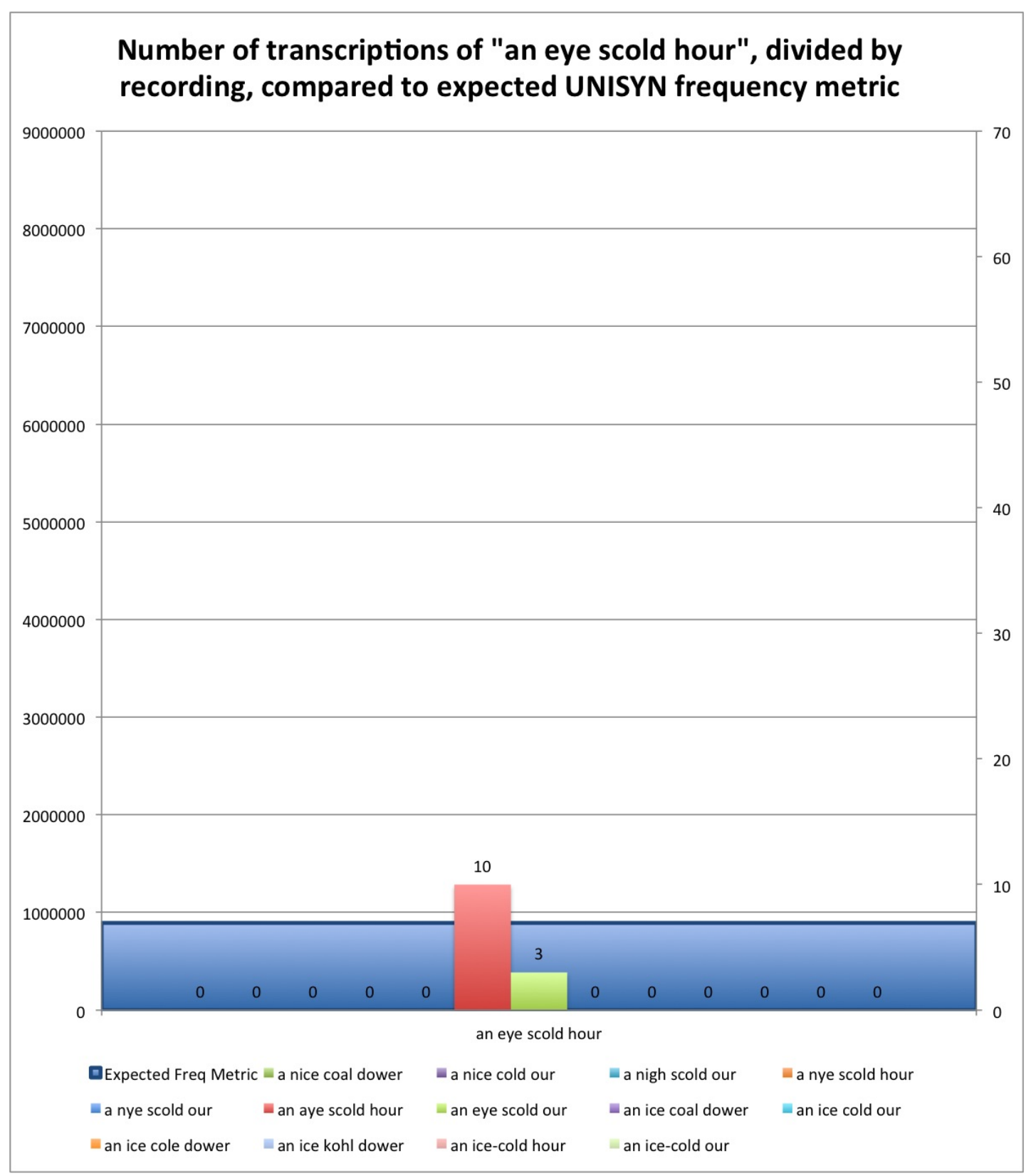

Figure 5.9: This graph represents all transcriptions of the phrase "an eye scold hour", divided into columns based on what recordings were transcribed as "an eye scold hour". The large blue bar in the background shows the predicted frequency metric for the phrase in question. 


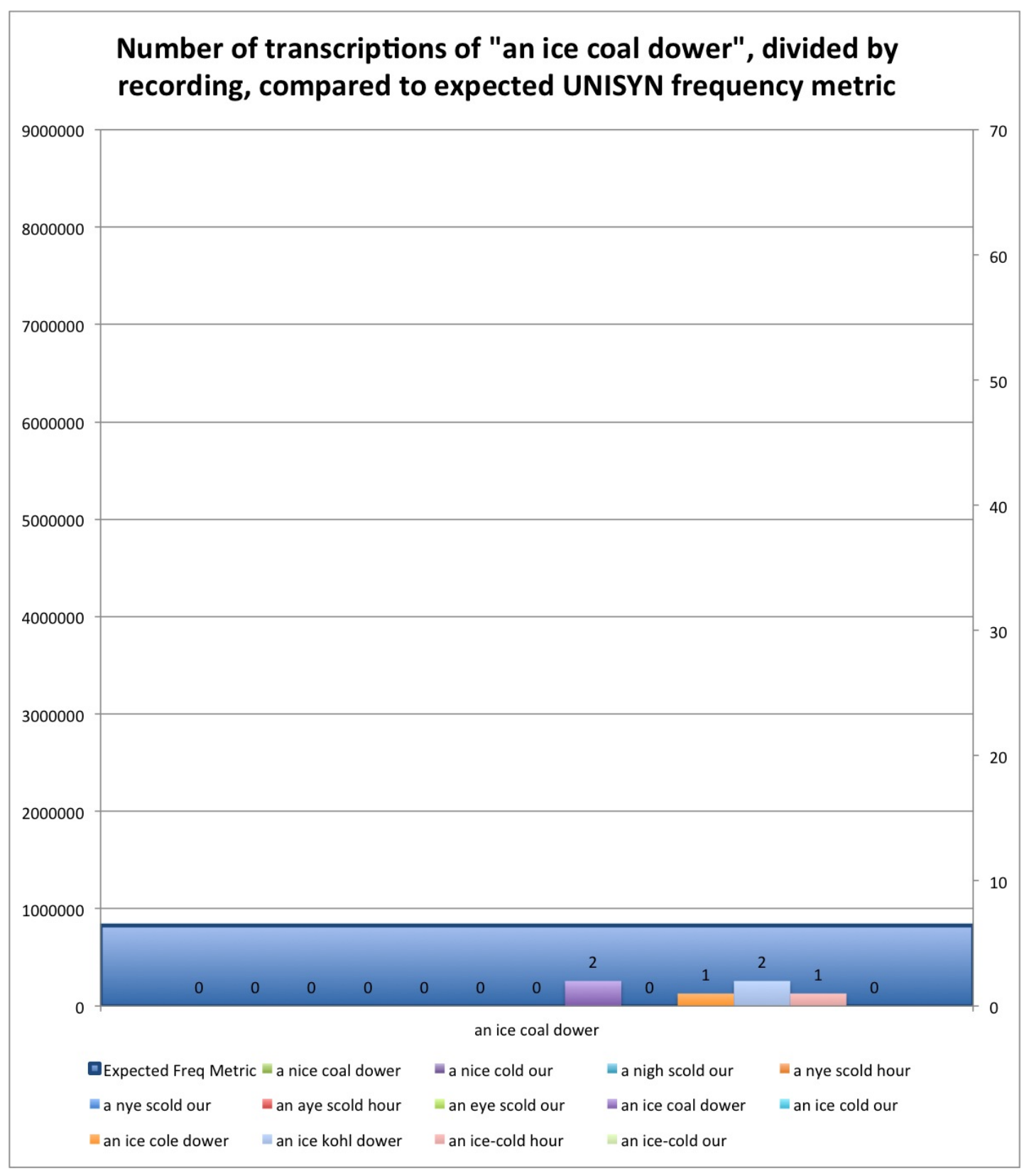

Figure 5.10: This graph represents all transcriptions of the phrase "an ice coal dower", divided into columns based on what recordings were transcribed as "an ice coal dower". The large blue bar in the background shows the predicted frequency metric for the phrase in question. 


\section{English-dominant countries}

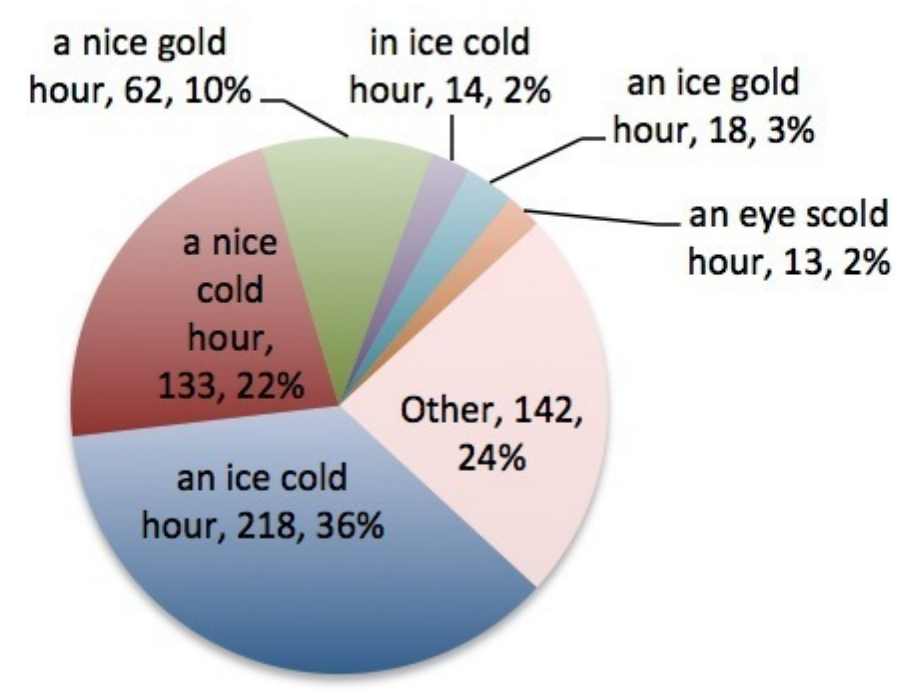

Figure 5.11: Pie Chart of transcriptions from countries that are primarily English-speaking.

\section{Non-Native English Countries}

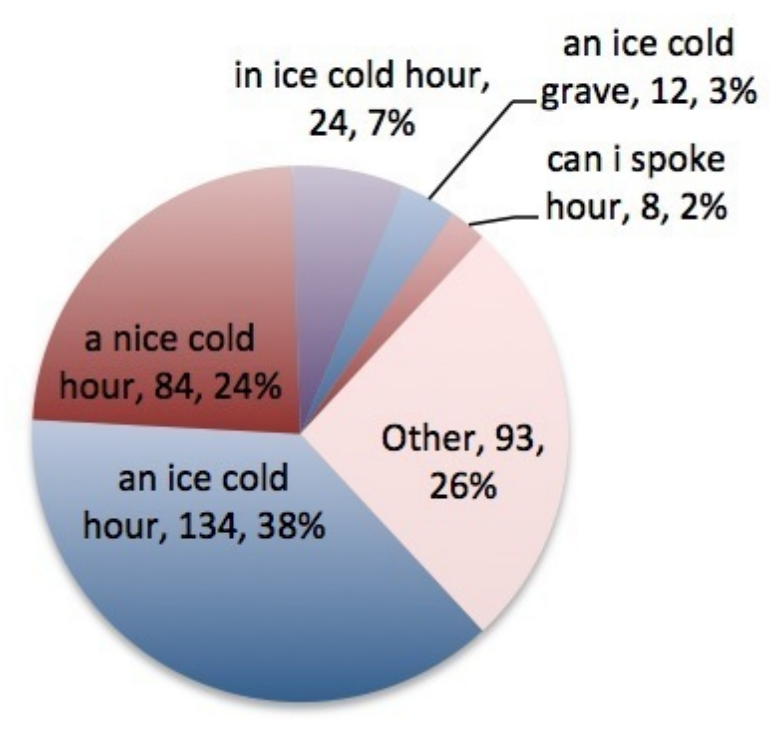

Figure 5.12: Pie Chart of transcriptions from countries that are Nonnative English speakers. 
1. The most common transcription for both is "an ice cold hour", with $36 \%$ native-speaker transcriptions ( 218 ) and $24 \%$ non-native ( 134 ).

2. The second most common transcription is also the same for both ("a nice cold hour"), but it accounts for a larger percentage of the non-native pie ( $24 \%$ compared to the native $22 \%$ ).

3. The third most common transcription differs for native and non-native speakers.

Native speakers transcribed "a nice gold hour" 62 times, accounting for 10 $\%$ of all native transcriptions. In comparison, only 1 non-native speaker transcribed that phrase, for a measly $0.2 \%$ of total non-native transcriptions.

This brings up an interesting data point - the third most popular transcription for native speakers barely shows up at all for non-native transcribers. There may be something about common phoneme substitution that native speakers pick up on that non-natives do not; specifically, a cold/gold merger.

The third most common transcription for non-native speakers is "in ice cold hour", which was transcribed 24 times, and makes up $7 \%$ of non-native transcriptions. This phrase was the fifth most common transcription for native speakers, with 14 transcriptions making up $2 \%$ of total transcriptions.

4. The fourth most common transcription for native speakers was "an ice gold hour", getting $3 \%$ of the total with 18 transcriptions (by 14 unique transcribers). This phrase was not transcribed by any non-native speakers. 
This exhibits the same phone/phoneme substitution that we saw with "a nice gold hour".

The fourth most common phrase for non-native speakers, "an ice cold grave", was only transcribed by one unique worker, and as such, is not going to be taken into serious consideration. The fifth most common phrase, "can I spoke hour", was also only transcribed by one unique worker, and so also cannot be taken into serious consideration.

\subsection{Deficiencies in The Oronyminator}

In some cases, our expected, UNISYN-derived phrase-frequency metric did not accurately line up with the observed transcription frequencies from our user studies. We believe that there are several possible reasons for this.

\subsubsection{Frequency Validity}

Our frequency source data from UNISYN ended up being less than satisfactory, due to several factors, deliniated below.

\section{Corpus Composition deficiencies}

The lack of quality phonemic frequency data is a known defeciency in our source dictionary, UNISYN. According to the authors of the UNISYN lexicon documentation:

It should be noted that the frequency field, as it was obtained from simple word lists, is not particularly reliable (emphasis mine).[25] 
The UNISYN frequency count is based upon a large but not exhaustive corpus of text. It has some particularly glaring deficiencies in the medical arena. We find this frustrating, because knowledge about common medical mondegreens could be used to prevent mistakes in patients' treatment plans[21]. Also, it meant that the word "colitis" wasn not in our dictionary, and we therefore could not use the example "the girl with colitis goes by" / "the girl with kaleidescope eyes".

\section{Homograph Differentiation}

Additionally, our source frequency data cannot and does not distinguish between words that may be homographs (that is, words that sound different but are spelled the same). This makes our program improperly weight some phrases over others.

For example, take the words for the animals "bucks" and "does". "Bucks" has a frequency of 1133, and "does" has a frequency of 508386. For comparison, "deer" has a frequency of 1896. You can see the relative scale of these in figure 5.13. It seems highly unlikely that the male and female labels for a species would be as or more common than the actual name of the species, given that we do not see this for sheep (sheep at 13572 , ewe at 186 ,and ram at 681) or horses (horse at 27559 , mare at 1055 , and stallion at 644 ). What is much more likely is that "bucks" is getting extra hits through its meaning as a slang synonym for dollars (dollars at 8927), and "does" is getting most of its frequency count for the third person present tense of the verb "to do". That seems very likely, given that the frequency for the singular "doe" is only 1077.

Future work on our project would benefit from using a dictionary with some way of distinguishing homographs when counting frequency. 


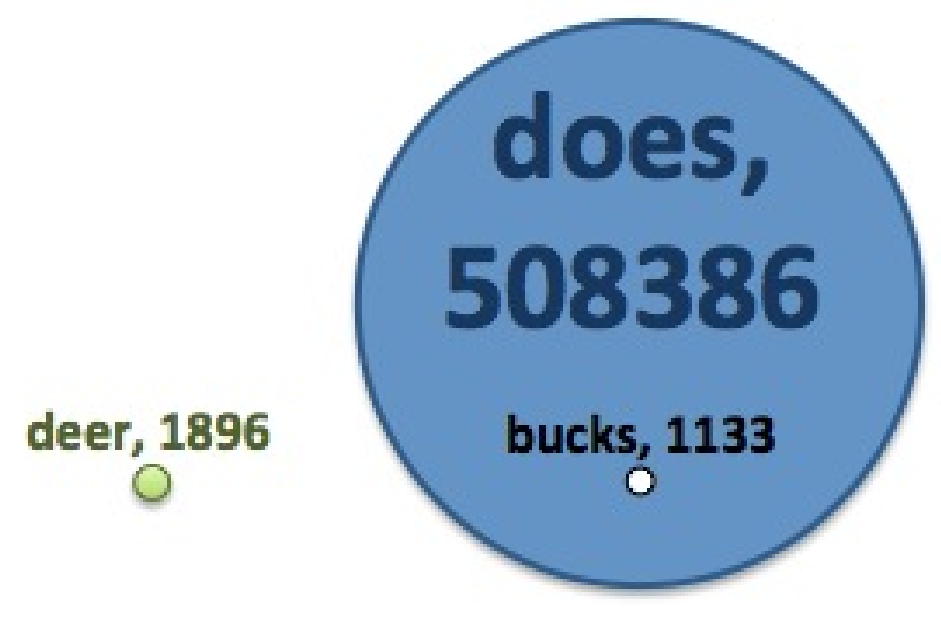

Figure 5.13: Bubble Chart comparison of Frequency for deer, does, and bucks

\section{Frequency Dictionary Tallying Methods}

In the future, we would also like to use word frequency values from a dictionary that takes a larger, more diverse dataset into its frequency count, such as the frequency lists from the Corpus of Contemporary American English[3]. The COCA corpus is entirely focused on word frequency, and as such, does not contain any phonetic data. However, it contains several different ways of determining frequency of words that overcome some of the shortcomings we ran into trying to compare the semantically-identical words 'a' and 'an'. ' $\mathrm{A}$ ' is found much more frequently than 'an', but both are just as common. In the UNISYN dictionary, we only have contextless frequency counts. In the COCA frequency dictionary, they keep two types of counts: one for how many times the word has been found total, and one for how many documents the word has been found in. This way, even though ' $a$ ' is found almost seven times as often than 'a' overall, we know that they are equally-familiar words, because they are both found in approximately $160 \mathrm{k}$ corpus entries[23]. 
As a proof of this concept, we created a sunburst diagram for "an ice cold hour" using COCA by-document frequency values (shown in figure 5.14), to compare it against the sunburst diagram created using UNISYN frequency values (shown in figure 5.15). The COCA-based sunburst, while still inaccurate, at least has a ratio of phrases beginning with "a" to "an" that is closer to the actual observed ratio (which can be seen back in figure 5.3).

A statistical analysis of the observed dataset frequencies versus a COCAderived frequency dataset, using a one-proportion $\mathrm{z}$ test, further proves that the COCA frequency values are a better match for the observed data than the UNISYN-derived frequencies were. Again using the top two observed transcriptions as our sample population, we take a look at the phrases "a nice cold hour" and "an ice cold hour" . The phrase "a nice cold hour" has a calculated COCA freq metric of 247719 , and had 125 actual transcriptions observed among people living in the United States. The phrase "an ice cold hour" has a calculated COCA freq metric of 227405, and had 191 actual transcriptions observed among people living in the United States. Therefore, the expected population is 475124 , and the observed population is 316 .

Given those population, the expected population proportion for "a nice cold hour" would be $247719 \div 475124$, or 0.4793 .

In our user study, we found that 125 people transcribed "a nice cold hour" , and 191 people transcribed "an ice cold hour" , for a ratio of 0.65 to 1, where "a nice cold hour" accounts for 39.56\% ( $\mathrm{p}=0.3956$ ) of the combined count.

Given the observed population proportion of 0.3956 and the expected population proportion 0.4793 , we did a one-proportion z test with an $\alpha$ of 0.01 . The $\mathrm{z}$ value returned was 3.0428 , meaning that the observed population proportion 


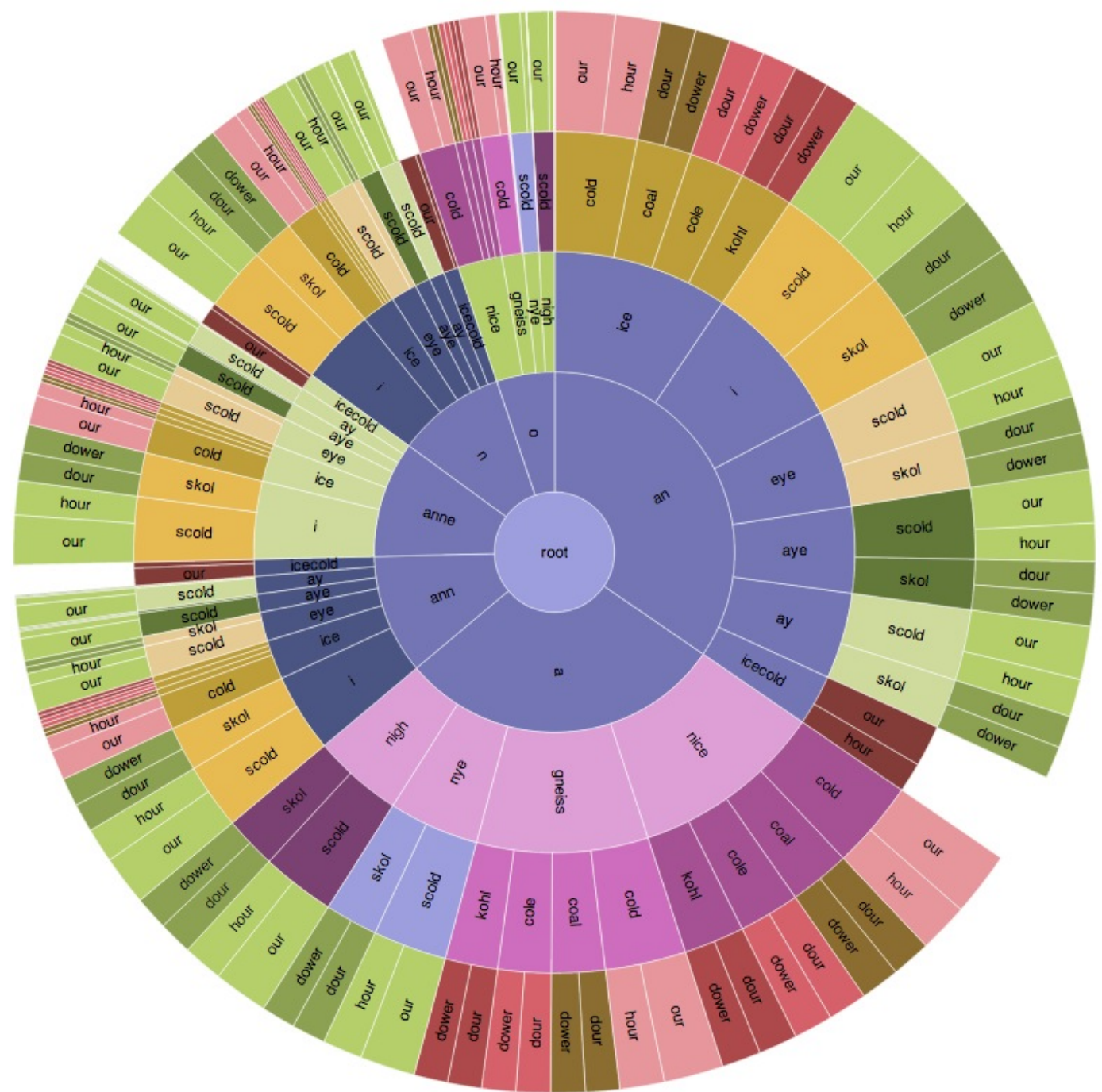

Size: Paths weighted by COCA freq metric

Figure 5.14: Sunburst diagram for "an ice cold hour" using COCA by-document freq metric 


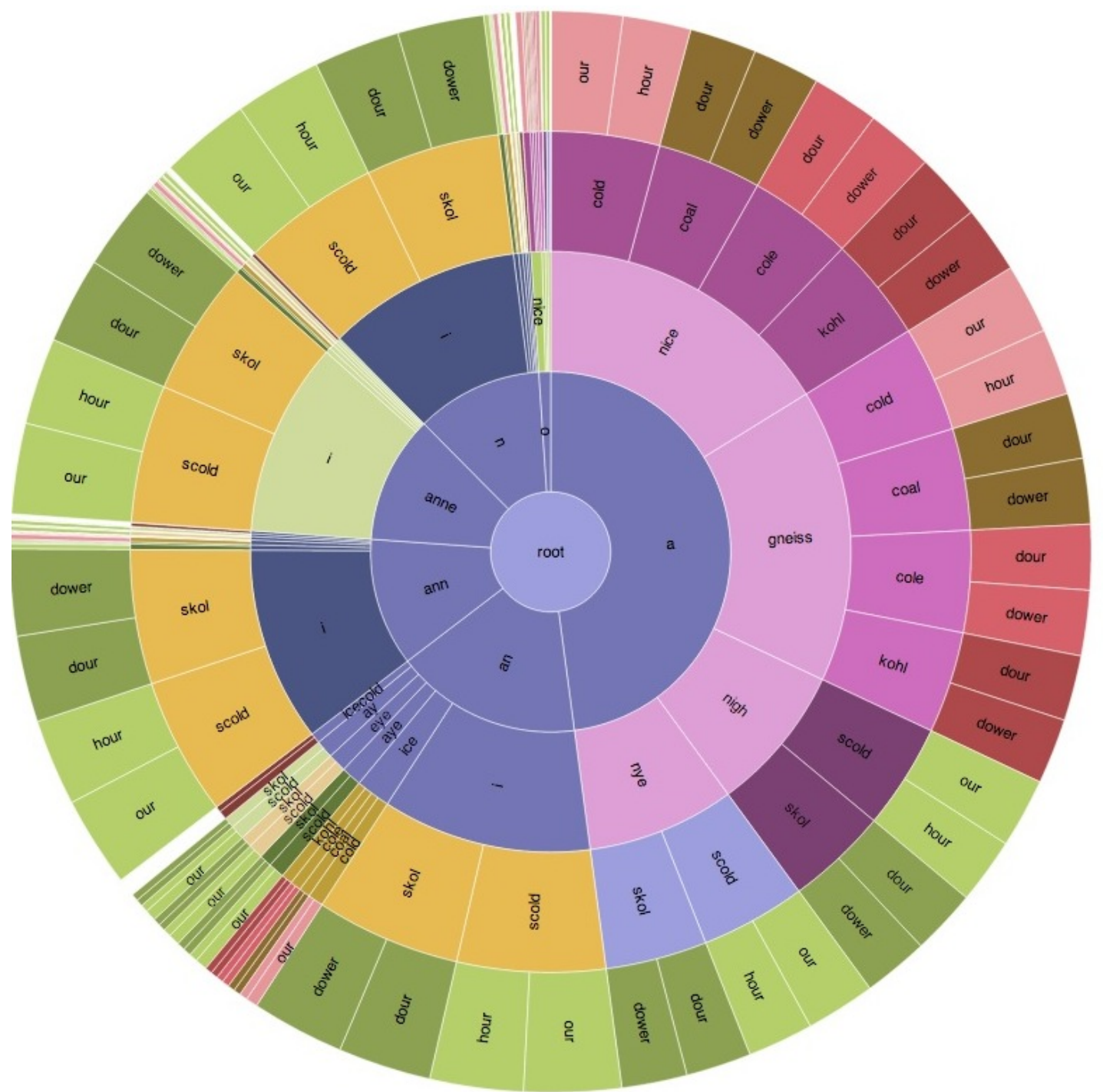

Size: Paths weighted by UNISYN freq metric

Figure 5.15: Sunburst diagram for "an ice cold hour" using UNISYN freq metric 
was 3.0428 standard deviations away from the expected population proportion. When we used this $\mathrm{z}$ value to compute a $\mathrm{p}$ value, we were left with a pvalue of 0.0023 , which is greater than our $\alpha$ of 0.01 . Therefore, there is an approximately $0.01 \%$ chance that the observed data could match the COCA predictions. While that is still not incredibly likely, it remains significantly more likely than the possibility of the UNISYN predictions being correct (which were 18.0971 standard deviations away from the expected population proportion, as calculated in 5.2.2. 


\subsection{Future Work}

\subsubsection{Higher-order frequency data}

Right now, our program only takes into account the frequency of standalone words, without taking their context into consideration. In the future, we would like to integrate n-grams into our program. N-grams are a probabilistic model of predicting the next item that will follow in a sequence, based upon frequencies of how often those $\mathrm{N}$ items occur in sequence in a corpus of text[12]. A word-level 4-gram, for example, would be a series of four words. Here are some 4-gram phrases, along with counts of how often they occur, from the Google Ngram corpus:

serve as the informational 41

serve as the infrastructure 500

serve as the initial 5331

serve as the initiating 125

serve as the initiation 63

serve as the initiator 81

serve as the injector 56

serve as the inlet 41

serve as the inner 87

serve as the input 1323

$[1]$

To give an example of what our frequency metric might look like if it incorporated n-grams, we looked up the historical n-gram occurence percentage of n-grams contained in oronyms of our main test phrase "an ice cold hour". As seen in figure 5.16, comparing the 3-grams "a nice cold" and "an ice cold" results in a fairly even split, though the latter phrase is slightly more likely to occur in modern-day settings. When we look at the 2-grams of that 3-gram, we see more 


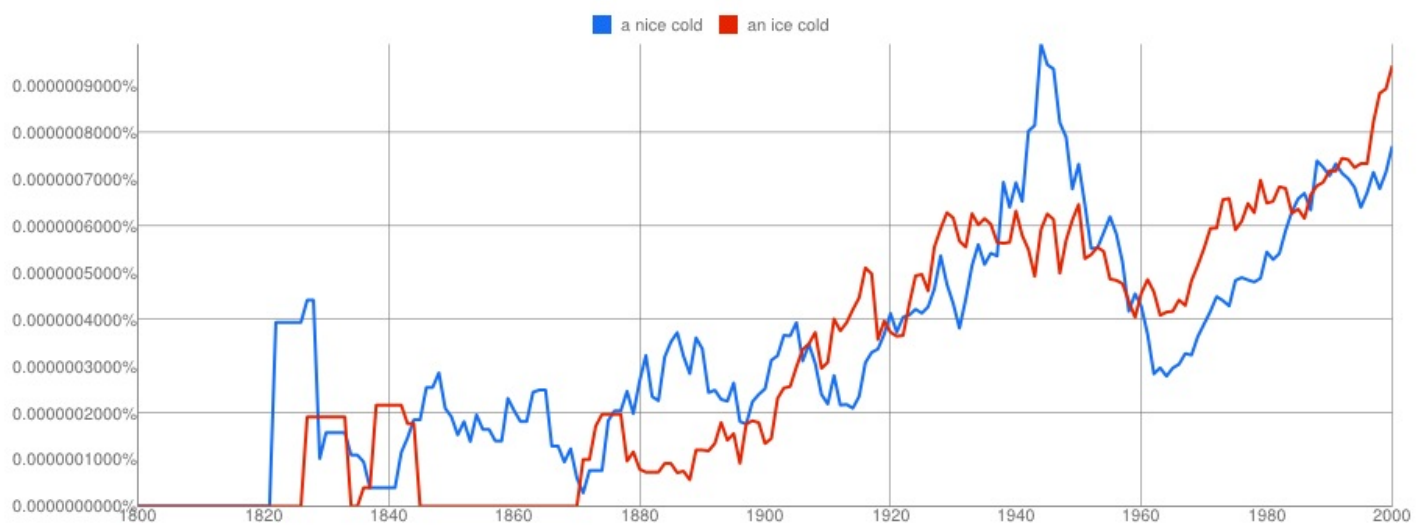

Figure 5.16: Historical N-gram data comparing the three-grams "a nice cold" and "an ice cold"

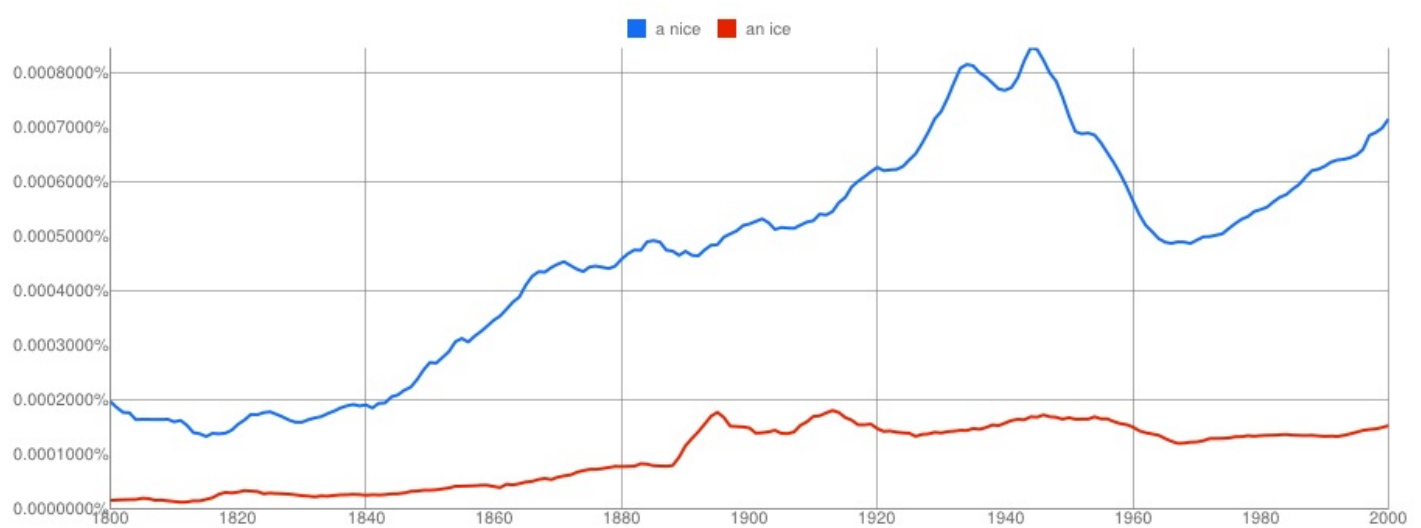

Figure 5.17: Historical N-gram data comparing the two-grams "a nice" and "an ice"

interesting trends. In figure 5.17, we see that "a nice" is consistently more frequently found in text than "an ice" is. However, when we compare the 2-grams "ice cold" and "nice cold", as we do in figure 5.18, we see that the phrase "ice cold" is leaps and bounds more likely to be encountered in everyday language.

Though we are happy with our findings, we believe that we could create even better likelihood metrics with the integration of several different orders of n-grams, and would suggest this for future work. However, if the final purpose of the oronyminator ends up being in the song lyric domain, everyday-usage 


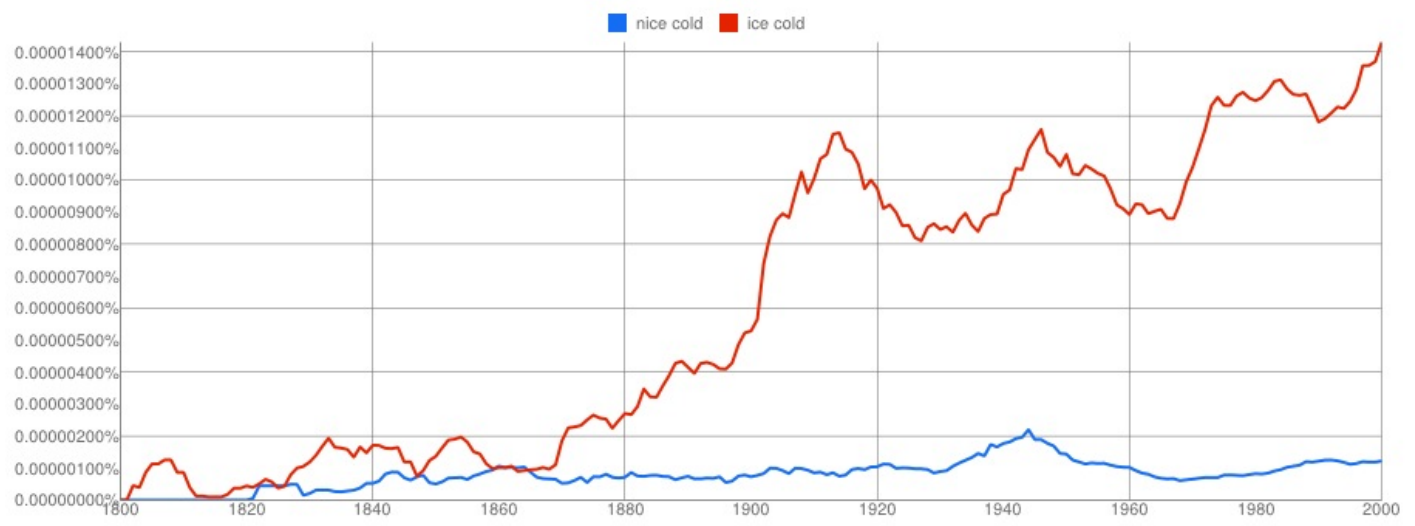

Figure 5.18: Historical N-gram data comparing the two-grams "nice cold" and "ice cold"

syntactical predictability may not be particularly relevant, due to the differences in vocabulary and grammar found in song lyrics versus regular prose.

\subsubsection{Phoneme swapping}

Often when speaking, humans substitute easier-to-say phones for more timeintensive phones. One of the main ways that this substitution occurs is through voiced/voiceless pairs. To voice a phone means to cause the vocal chords to vibrate. Voiced phones are singable, whereas voiceless phones are not. Voiceless phones are like a hiss, and simply direct streams of escaping air. Most consonant phonemes are part of a voice/voiceless pair, such as 't' and 'd' (The word "pretty", when spoken quickly, often uses a 'd' sound instead of a ' $t$ ' sound, because the phoneme for ' $d$ ' is easier to say). Phones are paired when the only differences between their pronuciation is the voicing, aka, when their manner of articulation (i.e. their manner of directing air during the sound), mouth end position, and mouth start position are the same (To view all phones in the SAMPA alphabet, along with enough information to determine whether they are pairs, see table C). In our project, we came across an example of phoneme swapping in the 
"cold" / "gold" transcriptions, which we went over in figure 5.7 and section 5.2.3. For future work, we suggest looking into phoneme swap pairings, and integrating the findings into the existing algorithm.

\subsubsection{Melody Matcher master project}

MisheardMe Oronyminator is a part of the greater Melody Matcher suite. Melody Matcher is a semi-automated music composition support program. It analyzes English lyrics along with a melody, and alerts the composer of the locations in the song where the lyrics are not deterministically understandable. Basically, it is grammar- and spell-check for songs.

Melody Matcher aims to replicate the human ability to identify lyrics in a song that are easily misheard.

\subsubsection{Target Audience and Goals}

This program is to be used as a compositional aid by anyone who wants to write songs and make them sound good, technically. It should allow the song writer to focus on more subjective criteria of what makes a song "good", because it will make the structural rules of lyric composition immediately apparent.

Our hope for this project is that it will be useful to burgeoning songwriters, who have the creative spark to make wonderfully poetic lyrics, but lack the "ear" to match their lyrics successfully to music. It should be particularly helpful to songwriters who place a high emphasis on understandability of lyrics (such as parody song writers, or lyricists for musical theater). 
Additionally, Melody Matcher will be useful for songwriters for whom English is a second language. While they may be a master lyricist in their native language, writing lyrics in English can be a particular challenge, since so much of lyricwriting is dependent upon knowing the cadence of the language one writes their lyrics in, and since English has no easily discernible rules for emphasis placement in words.

It will also be useful in the commercial space, for analyzing jingles. In order for a jingle to be effective, it must be deterministically and easily understandable, so that the message of the jingle gets across. By using Melody Matcher, jingle writers will be able to objectively measure this aspect of their output.

While MisheardMe Oronyminator only takes into account phonetics and frequencies, Melody Matcher analyzes the intelligibility of song lyrics by investigating several additional root causes:

- Lyric/Music emphasis mismatch, due to:

- Note intervals

- Phrase emphases

- Word emphases

- Word "cramming", due to:

- Syllable lengths that exceed that of note length

- Mouth movement delta time intervals

- Word misidentification, due to:

- Altered pronunciation of words 
- Phone similarity

* Voicing (voiced vs. voiceless)

* Beginning/end mouth positions

* Type (Plosive, Fricative, affricate, nasal, lateral, approximant, semivowel)

The fully-implemented Melody Matcher program will eventually take into account all of these causes of unintelligibility. 


\subsection{Conclusion}

In this paper, we have demonstrated MisheardMe Oronyminator, a computer program that takes in textual phrases in English, determines all oronyms for those phrases and then visualizes them using associated frequency information to indicate the likelihood of interpretation. We have demonstrated all four major functional parts: our custom phonetic dictionary, our command-line oronym generator, our OpenGL oronym-parse-tree visualization generator, and our Protovis sunburst diagrams. Our custom phonetic dictionary has some inconsistencies in word frenquency, due to the UNISYN source dictionary's frequency values not being generated from a well-sampled corpus. However, our program has no major structural flaws, and can be succesfully used for phrases with words that have frequencies on the same order of magnitude. Our command-line oronym generator successfully generates all oronyms that are exact phonetic matches for an orthographic phrase. The user studies that we did supported our generated phrases, if not our frequency metrics. Our oronym visualizations had two goals: one, to visually represent the likelihood of each oronym interpretation, visualized by scaling branches or arcs by phrase frequency values; and two, to exhibit orthographic phrases that may not have any exact oronyms, but have many dead-end, partial oronyms that could cause ambiguity. Our visualizations successfully accomplish both of those goals. 
Appendix A

\section{Implementation Details}




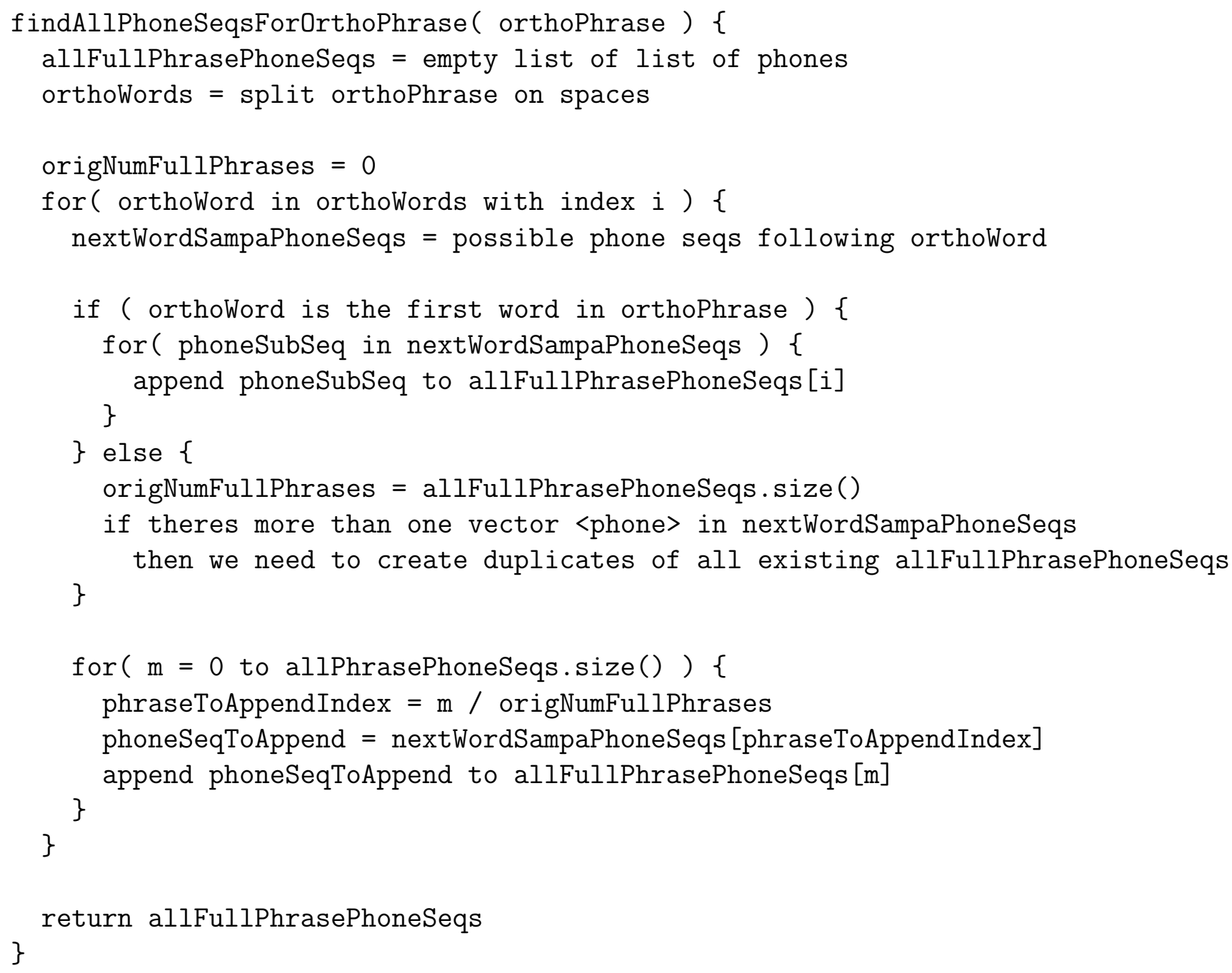

Figure A.1: Algorithm to get all phonetic sequences for an orthographic phrase. 


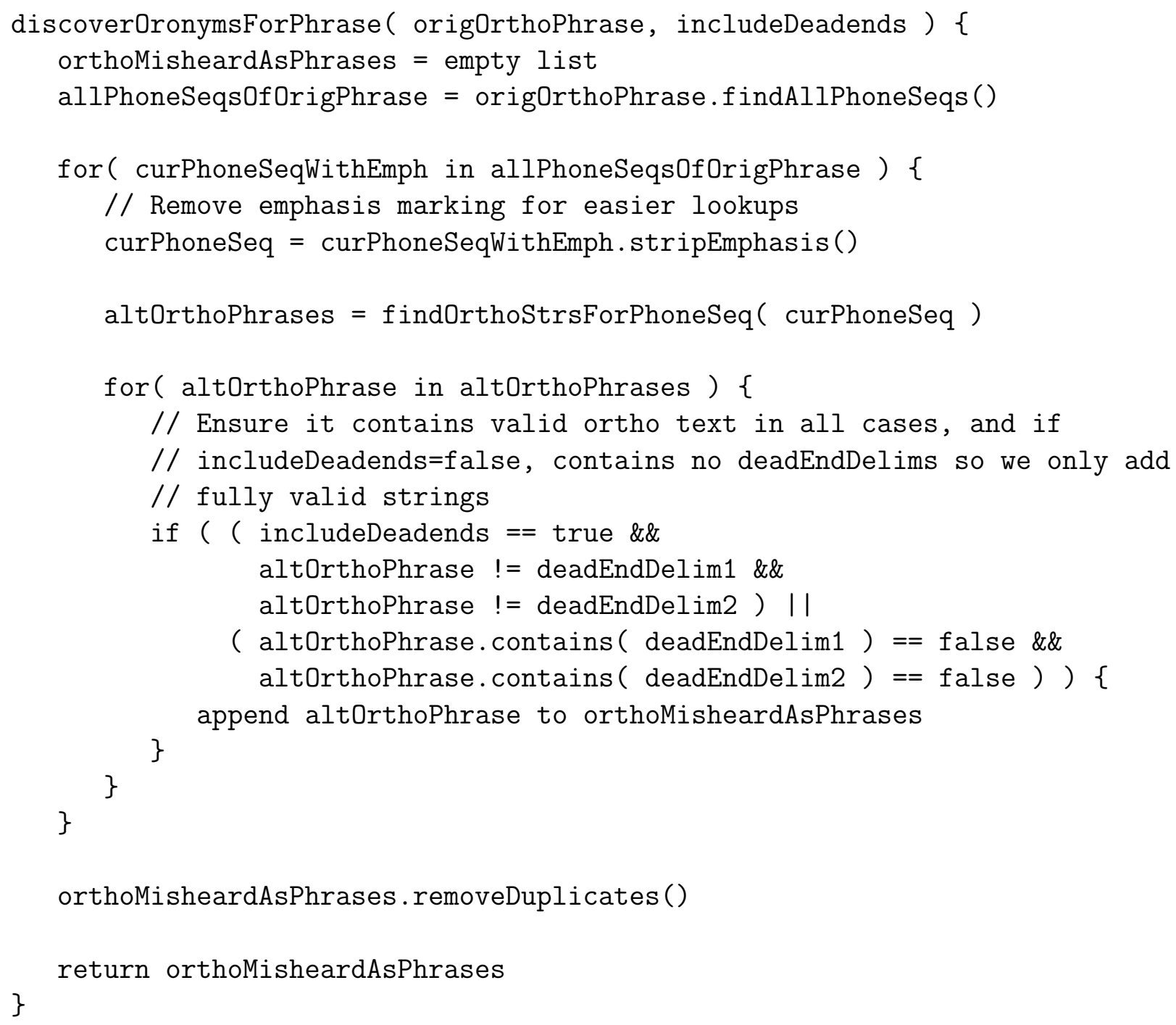

Figure A.2: Algorithm to get all oronyms for an orthographic phrase. 


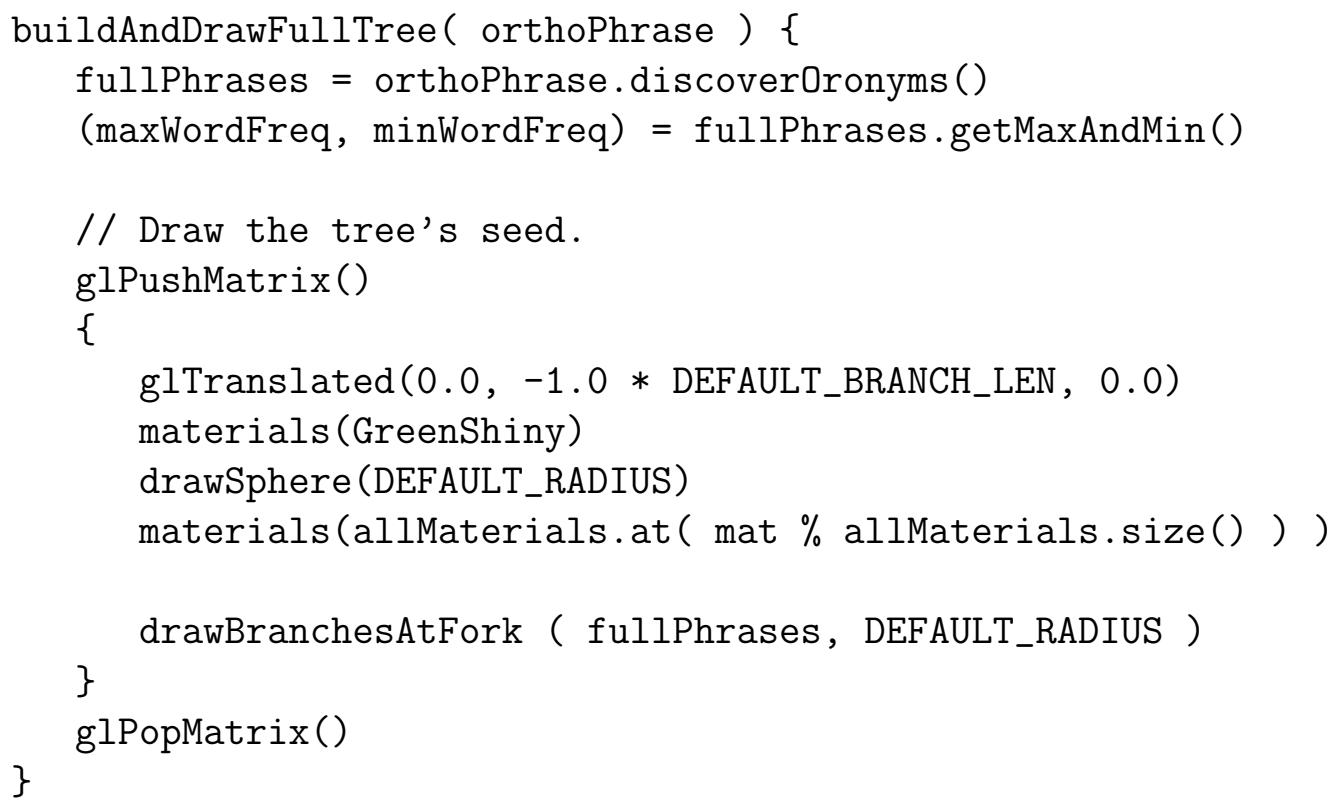

Figure A.3: Given an orthographic phrase, this function prepares to draw the tree 


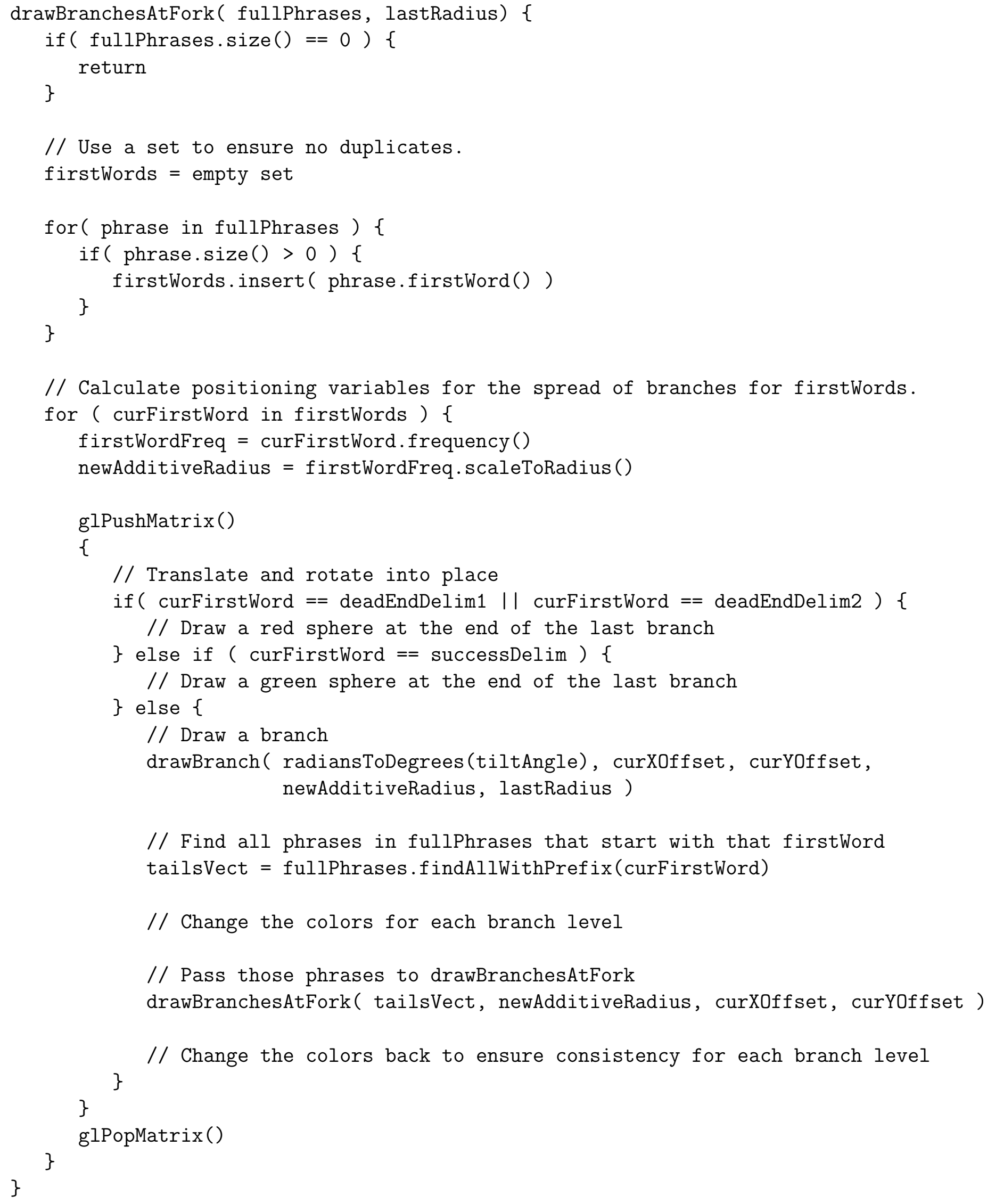

Figure A.4: This is the function that facilitates the in-time drawing of the tree as we parse though our oronyms possibilities 


\section{Appendix B}

\section{Oronym Tables}

\begin{tabular}{cc|cccccccc}
\hline phrase & total freq & word1 & freq1 & word2 & freq2 & word3 & freq3 & word4 & freq4 \\
\hline on i scold our & 13185760 & on & 2774243 & i & 9937877 & scold & 217 & our & 473423 \\
\hline on i scold hour & 12784150 & on & 2774243 & i & 9937877 & scold & 217 & hour & 71813 \\
\hline on i skol dour & 12712244 & on & 2774243 & i & 9937877 & skol & 5 & dour & 119 \\
\hline on i skol dower & 12712217 & on & 2774243 & i & 9937877 & skol & 5 & dower & 92 \\
\hline an i scold our & 11205686 & an & 794169 & i & 9937877 & scold & 217 & our & 473423 \\
\hline an i scold hour & 10804076 & an & 794169 & i & 9937877 & scold & 217 & hour & 71813 \\
\hline an i skol dour & 10732170 & an & 794169 & i & 9937877 & skol & 5 & dour & 119 \\
\hline an i skol dower & 10732143 & an & 794169 & i & 9937877 & skol & 5 & dower & 92 \\
\hline 'n' i scold our & 10411517 & 'n' & 0 & i & 9937877 & scold & 217 & our & 473423 \\
\hline 'n' i scold hour & 10009907 & 'n' & 0 & i & 9937877 & scold & 217 & hour & 71813 \\
\hline 'n' i skol dour & 9938001 & 'n' & 0 & i & 9937877 & skol & 5 & dour & 119 \\
\hline 'n' i skol dower & 9937974 & 'n' & 0 & i & 9937877 & skol & 5 & dower & 92 \\
\hline a nice cold our & 8253272 & a & 7536297 & nice & 190708 & cold & 52844 & our & 473423 \\
\hline \multicolumn{2}{c}{} & & & & & & & Continued on next page \\
\hline
\end{tabular}


Table B.1 - continued from previous page

\begin{tabular}{|c|c|c|c|c|c|c|c|c|c|}
\hline phrase & total freq & word1 & freq1 & word2 & freq2 & word3 & freq3 & word4 & freq4 \\
\hline a niece cold our & 8064257 & $\mathrm{a}$ & 7536297 & niece & 1693 & cold & 52844 & our & 473423 \\
\hline a gneiss cold our & 8062585 & $\mathrm{a}$ & 7536297 & gneiss & 21 & cold & 52844 & our & 473423 \\
\hline a ne scold our & 8017040 & $\mathrm{a}$ & 7536297 & ne & 7103 & scold & 217 & our & 473423 \\
\hline a knee scold our & 8016076 & $\mathrm{a}$ & 7536297 & knee & 6139 & scold & 217 & our & 473423 \\
\hline a nigh scold our & 8011331 & $\mathrm{a}$ & 7536297 & nigh & 1394 & scold & 217 & our & 473423 \\
\hline a nye scold our & 8009974 & $\mathrm{a}$ & 7536297 & nye & 37 & scold & 217 & our & 473423 \\
\hline a nice cold hour & 7851662 & $\mathrm{a}$ & 7536297 & nice & 190708 & cold & 52844 & hour & 71813 \\
\hline a nice coal dour & 7747572 & $\mathrm{a}$ & 7536297 & nice & 190708 & coal & 20448 & dour & 119 \\
\hline a nice coal dower & 7747545 & $\mathrm{a}$ & 7536297 & nice & 190708 & coal & 20448 & dower & 92 \\
\hline a nice cole dour & 7729197 & $\mathrm{a}$ & 7536297 & nice & 190708 & cole & 2073 & dour & 119 \\
\hline a nice cole dower & 7729170 & $\mathrm{a}$ & 7536297 & nice & 190708 & cole & 2073 & dower & 92 \\
\hline a nice kohl dour & 7728036 & $\mathrm{a}$ & 7536297 & nice & 190708 & kohl & 912 & dour & 119 \\
\hline a nice kohl dower & 7728009 & $\mathrm{a}$ & 7536297 & nice & 190708 & kohl & 912 & dower & 92 \\
\hline a niece cold hour & 7662647 & $\mathrm{a}$ & 7536297 & niece & 1693 & cold & 52844 & hour & 71813 \\
\hline a gneiss cold hour & 7660975 & $\mathrm{a}$ & 7536297 & gneiss & 21 & cold & 52844 & hour & 71813 \\
\hline a ne scold hour & 7615430 & $\mathrm{a}$ & 7536297 & ne & 7103 & scold & 217 & hour & 71813 \\
\hline a knee scold hour & 7614466 & $\mathrm{a}$ & 7536297 & knee & 6139 & scold & 217 & hour & 71813 \\
\hline a nigh scold hour & 7609721 & $\mathrm{a}$ & 7536297 & nigh & 1394 & scold & 217 & hour & 71813 \\
\hline a nye scold hour & 7608364 & a & 7536297 & nye & 37 & scold & 217 & hour & 71813 \\
\hline a niece coal dour & 7558557 & $\mathrm{a}$ & 7536297 & niece & 1693 & coal & 20448 & dour & 119 \\
\hline a niece coal dower & 7558530 & $\mathrm{a}$ & 7536297 & niece & 1693 & coal & 20448 & dower & 92 \\
\hline a gneiss coal dour & 7556885 & $\mathrm{a}$ & 7536297 & gneiss & 21 & coal & 20448 & dour & 119 \\
\hline & & & & & & & \multicolumn{3}{|c|}{ Continued on next page } \\
\hline
\end{tabular}


Table B.1 - continued from previous page

\begin{tabular}{|c|c|c|c|c|c|c|c|c|c|}
\hline phrase & total freq & word1 & freq1 & word2 & freq2 & word3 & freq3 & word4 & freq4 \\
\hline a gneiss coal dower & 7556858 & $\mathrm{a}$ & 7536297 & gneiss & 21 & coal & 20448 & dower & 92 \\
\hline a ne skol dour & 7543524 & $\mathrm{a}$ & 7536297 & ne & 7103 & skol & 5 & dour & 119 \\
\hline a ne skol dower & 7543497 & $\mathrm{a}$ & 7536297 & ne & 7103 & skol & 5 & dower & 92 \\
\hline a knee skol dour & 7542560 & $\mathrm{a}$ & 7536297 & knee & 6139 & skol & 5 & dour & 119 \\
\hline a knee skol dower & 7542533 & $\mathrm{a}$ & 7536297 & knee & 6139 & skol & 5 & dower & 92 \\
\hline a niece cole dour & 7540182 & $\mathrm{a}$ & 7536297 & niece & 1693 & cole & 2073 & dour & 119 \\
\hline a niece cole dower & 7540155 & $\mathrm{a}$ & 7536297 & niece & 1693 & cole & 2073 & dower & 92 \\
\hline a niece kohl dour & 7539021 & $\mathrm{a}$ & 7536297 & niece & 1693 & kohl & 912 & dour & 119 \\
\hline a niece kohl dower & 7538994 & $\mathrm{a}$ & 7536297 & niece & 1693 & kohl & 912 & dower & 92 \\
\hline a gneiss cole dour & 7538510 & $\mathrm{a}$ & 7536297 & gneiss & 21 & cole & 2073 & dour & 119 \\
\hline a gneiss cole dower & 7538483 & $\mathrm{a}$ & 7536297 & gneiss & 21 & cole & 2073 & dower & 92 \\
\hline a nigh skol dour & 7537815 & $\mathrm{a}$ & 7536297 & nigh & 1394 & skol & 5 & dour & 119 \\
\hline a nigh skol dower & 7537788 & $\mathrm{a}$ & 7536297 & nigh & 1394 & skol & 5 & dower & 92 \\
\hline a gneiss kohl dour & 7537349 & $\mathrm{a}$ & 7536297 & gneiss & 21 & kohl & 912 & dour & 119 \\
\hline a gneiss kohl dower & 7537322 & $\mathrm{a}$ & 7536297 & gneiss & 21 & kohl & 912 & dower & 92 \\
\hline a nye skol dour & 7536458 & $\mathrm{a}$ & 7536297 & nye & 37 & skol & 5 & dour & 119 \\
\hline a nye skol dower & 7536431 & $\mathrm{a}$ & 7536297 & nye & 37 & skol & 5 & dower & 92 \\
\hline on aye scold our & 3378386 & on & 2774243 & aye & 130503 & scold & 217 & our & 473423 \\
\hline on e scold our & 3356846 & on & 2774243 & e & 108963 & scold & 217 & our & 473423 \\
\hline on ice cold our & 3312712 & on & 2774243 & ice & 12202 & cold & 52844 & our & 473423 \\
\hline on eye scold our & 3274633 & on & 2774243 & eye & 26750 & scold & 217 & our & 473423 \\
\hline on ay scold our & 3254516 & on & 2774243 & ay & 6633 & scold & 217 & our & 473423 \\
\hline & & & & & & & \multicolumn{3}{|c|}{ Continued on next page } \\
\hline
\end{tabular}


Table B.1 - continued from previous page

\begin{tabular}{|c|c|c|c|c|c|c|c|c|c|}
\hline phrase & total freq & word1 & freq1 & word2 & freq2 & word3 & freq3 & word4 & freq4 \\
\hline on ice-cold our & 3247715 & on & 2774243 & ice-cold & 49 & our & 473423 & & \\
\hline on aye scold hour & 2976776 & on & 2774243 & aye & 130503 & scold & 217 & hour & 71813 \\
\hline on e scold hour & 2955236 & on & 2774243 & e & 108963 & scold & 217 & hour & 71813 \\
\hline on ice cold hour & 2911102 & on & 2774243 & ice & 12202 & cold & 52844 & hour & 71813 \\
\hline on aye skol dour & 2904870 & on & 2774243 & aye & 130503 & skol & 5 & dour & 119 \\
\hline on aye skol dower & 2904843 & on & 2774243 & aye & 130503 & skol & 5 & dower & 92 \\
\hline on e skol dour & 2883330 & on & 2774243 & e & 108963 & skol & 5 & dour & 119 \\
\hline on e skol dower & 2883303 & on & 2774243 & $\mathrm{e}$ & 108963 & skol & 5 & dower & 92 \\
\hline on eye scold hour & 2873023 & on & 2774243 & eye & 26750 & scold & 217 & hour & 71813 \\
\hline on ay scold hour & 2852906 & on & 2774243 & ay & 6633 & scold & 217 & hour & 71813 \\
\hline on ice-cold hour & 2846105 & on & 2774243 & ice-cold & 49 & hour & 71813 & & \\
\hline on ice coal dour & 2807012 & on & 2774243 & ice & 12202 & coal & 20448 & dour & 119 \\
\hline on ice coal dower & 2806985 & on & 2774243 & ice & 12202 & coal & 20448 & dower & 92 \\
\hline on eye skol dour & 2801117 & on & 2774243 & eye & 26750 & skol & 5 & dour & 119 \\
\hline on eye skol dower & 2801090 & on & 2774243 & eye & 26750 & skol & 5 & dower & 92 \\
\hline on ice cole dour & 2788637 & on & 2774243 & ice & 12202 & cole & 2073 & dour & 119 \\
\hline on ice cole dower & 2788610 & on & 2774243 & ice & 12202 & cole & 2073 & dower & 92 \\
\hline on ice kohl dour & 2787476 & on & 2774243 & ice & 12202 & kohl & 912 & dour & 119 \\
\hline on ice kohl dower & 2787449 & on & 2774243 & ice & 12202 & kohl & 912 & dower & 92 \\
\hline on ay skol dour & 2781000 & on & 2774243 & ay & 6633 & skol & 5 & dour & 119 \\
\hline on ay skol dower & 2780973 & on & 2774243 & ay & 6633 & skol & 5 & dower & 92 \\
\hline an aye scold our & 1398312 & an & 794169 & aye & 130503 & scold & 217 & our & 473423 \\
\hline & & & & & & & \multicolumn{3}{|c|}{ Continued on next page } \\
\hline
\end{tabular}


Table B.1 - continued from previous page

\begin{tabular}{|c|c|c|c|c|c|c|c|c|c|}
\hline phrase & total freq & word1 & freq1 & word2 & freq2 & word3 & freq3 & word4 & freq4 \\
\hline an e scold our & 1376772 & an & 794169 & e & 108963 & scold & 217 & our & 473423 \\
\hline an ice cold our & 1332638 & an & 794169 & ice & 12202 & cold & 52844 & our & 473423 \\
\hline an eye scold our & 1294559 & an & 794169 & eye & 26750 & scold & 217 & our & 473423 \\
\hline an ay scold our & 1274442 & an & 794169 & ay & 6633 & scold & 217 & our & 473423 \\
\hline an ice-cold our & 1267641 & an & 794169 & ice-cold & 49 & our & 473423 & & \\
\hline an aye scold hour & 996702 & an & 794169 & aye & 130503 & scold & 217 & hour & 71813 \\
\hline an e scold hour & 975162 & an & 794169 & e & 108963 & scold & 217 & hour & 71813 \\
\hline ah nice cold our & 946271 & ah & 229296 & nice & 190708 & cold & 52844 & our & 473423 \\
\hline an ice cold hour & 931028 & an & 794169 & ice & 12202 & cold & 52844 & hour & 71813 \\
\hline an aye skol dour & 924796 & an & 794169 & aye & 130503 & skol & 5 & dour & 119 \\
\hline an aye skol dower & 924769 & an & 794169 & aye & 130503 & skol & 5 & dower & 92 \\
\hline an e skol dour & 903256 & an & 794169 & e & 108963 & skol & 5 & dour & 119 \\
\hline an e skol dower & 903229 & an & 794169 & $\mathrm{e}$ & 108963 & skol & 5 & dower & 92 \\
\hline an eye scold hour & 892949 & an & 794169 & eye & 26750 & scold & 217 & hour & 71813 \\
\hline an ay scold hour & 872832 & an & 794169 & ay & 6633 & scold & 217 & hour & 71813 \\
\hline an ice-cold hour & 866031 & an & 794169 & ice-cold & 49 & hour & 71813 & & \\
\hline an ice coal dour & 826938 & an & 794169 & ice & 12202 & coal & 20448 & dour & 119 \\
\hline an ice coal dower & 826911 & an & 794169 & ice & 12202 & coal & 20448 & dower & 92 \\
\hline an eye skol dour & 821043 & an & 794169 & eye & 26750 & skol & 5 & dour & 119 \\
\hline an eye skol dower & 821016 & an & 794169 & eye & 26750 & skol & 5 & dower & 92 \\
\hline an ice cole dour & 808563 & an & 794169 & ice & 12202 & cole & 2073 & dour & 119 \\
\hline an ice cole dower & 808536 & an & 794169 & ice & 12202 & cole & 2073 & dower & 92 \\
\hline
\end{tabular}


Table B.1 - continued from previous page

\begin{tabular}{|c|c|c|c|c|c|c|c|c|c|}
\hline phrase & total freq & word1 & freq1 & word2 & freq2 & word3 & freq3 & word 4 & freq4 \\
\hline an ice kohl dour & 807402 & an & 794169 & ice & 12202 & kohl & 912 & dour & 119 \\
\hline an ice kohl dower & 807375 & an & 794169 & ice & 12202 & kohl & 912 & dower & 92 \\
\hline an ay skol dour & 800926 & an & 794169 & ay & 6633 & skol & 5 & dour & 119 \\
\hline an ay skol dower & 800899 & an & 794169 & ay & 6633 & skol & 5 & dower & 92 \\
\hline eh nice cold our & 783938 & eh & 66963 & nice & 190708 & cold & 52844 & our & 473423 \\
\hline ah niece cold our & 757256 & ah & 229296 & niece & 1693 & cold & 52844 & our & 473423 \\
\hline ah gneiss cold our & 755584 & $\mathrm{ah}$ & 229296 & gneiss & 21 & cold & 52844 & our & 473423 \\
\hline et nice cold our & 723706 & et & 6731 & nice & 190708 & cold & 52844 & our & 473423 \\
\hline o' nice cold our & 717438 & $\mathrm{O}^{\prime}$ & 463 & nice & 190708 & cold & 52844 & our & 473423 \\
\hline ah ne scold our & 710039 & ah & 229296 & ne & 7103 & scold & 217 & our & 473423 \\
\hline ah knee scold our & 709075 & ah & 229296 & knee & 6139 & scold & 217 & our & 473423 \\
\hline ah nigh scold our & 704330 & ah & 229296 & nigh & 1394 & scold & 217 & our & 473423 \\
\hline ah nye scold our & 702973 & ah & 229296 & nye & 37 & scold & 217 & our & 473423 \\
\hline 'n' aye scold our & 604143 & 'n' & 0 & aye & 130503 & scold & 217 & our & 473423 \\
\hline eh niece cold our & 594923 & eh & 66963 & niece & 1693 & cold & 52844 & our & 473423 \\
\hline eh gneiss cold our & 593251 & eh & 66963 & gneiss & 21 & cold & 52844 & our & 473423 \\
\hline 'n' e scold our & 582603 & 'n' & 0 & e & 108963 & scold & 217 & our & 473423 \\
\hline eh ne scold our & 547706 & eh & 66963 & ne & 7103 & scold & 217 & our & 473423 \\
\hline eh knee scold our & 546742 & eh & 66963 & knee & 6139 & scold & 217 & our & 473423 \\
\hline ah nice cold hour & 544661 & $\mathrm{ah}$ & 229296 & nice & 190708 & cold & 52844 & hour & 71813 \\
\hline eh nigh scold our & 541997 & eh & 66963 & nigh & 1394 & scold & 217 & our & 473423 \\
\hline eh nye scold our & 540640 & eh & 66963 & nye & 37 & scold & 217 & our & 473423 \\
\hline & & & & & & & \multicolumn{3}{|c|}{ Continued on next page } \\
\hline
\end{tabular}


Table B.1 - continued from previous page

\begin{tabular}{|c|c|c|c|c|c|c|c|c|c|}
\hline phrase & total freq & word1 & freq1 & word2 & freq2 & word3 & freq3 & word4 & freq4 \\
\hline 'n' ice cold our & 538469 & 'n' & 0 & ice & 12202 & cold & 52844 & our & 473423 \\
\hline et niece cold our & 534691 & et & 6731 & niece & 1693 & cold & 52844 & our & 473423 \\
\hline et gneiss cold our & 533019 & et & 6731 & gneiss & 21 & cold & 52844 & our & 473423 \\
\hline o' niece cold our & 528423 & o' & 463 & niece & 1693 & cold & 52844 & our & 473423 \\
\hline o' gneiss cold our & 526751 & $\mathrm{o}^{\prime}$ & 463 & gneiss & 21 & cold & 52844 & our & 473423 \\
\hline 'n' eye scold our & 500390 & 'n' & 0 & eye & 26750 & scold & 217 & our & 473423 \\
\hline et ne scold our & 487474 & et & 6731 & ne & 7103 & scold & 217 & our & 473423 \\
\hline et knee scold our & 486510 & et & 6731 & knee & 6139 & scold & 217 & our & 473423 \\
\hline et nigh scold our & 481765 & et & 6731 & nigh & 1394 & scold & 217 & our & 473423 \\
\hline o' ne scold our & 481206 & $\mathrm{o}^{\prime}$ & 463 & ne & 7103 & scold & 217 & our & 473423 \\
\hline et nye scold our & 480408 & et & 6731 & nye & 37 & scold & 217 & our & 473423 \\
\hline 'n' ay scold our & 480273 & 'n' & 0 & ay & 6633 & scold & 217 & our & 473423 \\
\hline o' knee scold our & 480242 & $\mathrm{o}^{\prime}$ & 463 & knee & 6139 & scold & 217 & our & 473423 \\
\hline o' nigh scold our & 475497 & $\mathrm{o}^{\prime}$ & 463 & nigh & 1394 & scold & 217 & our & 473423 \\
\hline o' nye scold our & 474140 & o' & 463 & nye & 37 & scold & 217 & our & 473423 \\
\hline 'n' ice-cold our & 473472 & 'n' & 0 & ice-cold & 49 & our & 473423 & & \\
\hline ah nice coal dour & 440571 & $\mathrm{ah}$ & 229296 & nice & 190708 & coal & 20448 & dour & 119 \\
\hline ah nice coal dower & 440544 & ah & 229296 & nice & 190708 & coal & 20448 & dower & 92 \\
\hline ah nice cole dour & 422196 & ah & 229296 & nice & 190708 & cole & 2073 & dour & 119 \\
\hline ah nice cole dower & 422169 & $\mathrm{ah}$ & 229296 & nice & 190708 & cole & 2073 & dower & 92 \\
\hline ah nice kohl dour & 421035 & ah & 229296 & nice & 190708 & kohl & 912 & dour & 119 \\
\hline ah nice kohl dower & 421008 & ah & 229296 & nice & 190708 & kohl & 912 & dower & 92 \\
\hline & & & & & & & \multicolumn{3}{|c|}{ Continued on next page } \\
\hline
\end{tabular}


Table B.1 - continued from previous page

\begin{tabular}{|c|c|c|c|c|c|c|c|c|c|}
\hline phrase & total freq & word1 & freq1 & word2 & freq2 & word3 & freq3 & word4 & freq4 \\
\hline eh nice cold hour & 382328 & eh & 66963 & nice & 190708 & cold & 52844 & hour & 71813 \\
\hline ah niece cold hour & 355646 & $\mathrm{ah}$ & 229296 & niece & 1693 & cold & 52844 & hour & 71813 \\
\hline ah gneiss cold hour & 353974 & $\mathrm{ah}$ & 229296 & gneiss & 21 & cold & 52844 & hour & 71813 \\
\hline et nice cold hour & 322096 & et & 6731 & nice & 190708 & cold & 52844 & hour & 71813 \\
\hline o' nice cold hour & 315828 & $\mathrm{o}^{\prime}$ & 463 & nice & 190708 & cold & 52844 & hour & 71813 \\
\hline ah ne scold hour & 308429 & $\mathrm{ah}$ & 229296 & ne & 7103 & scold & 217 & hour & 71813 \\
\hline ah knee scold hour & 307465 & $\mathrm{ah}$ & 229296 & knee & 6139 & scold & 217 & hour & 71813 \\
\hline ah nigh scold hour & 302720 & ah & 229296 & nigh & 1394 & scold & 217 & hour & 71813 \\
\hline ah nye scold hour & 301363 & ah & 229296 & nye & 37 & scold & 217 & hour & 71813 \\
\hline eh nice coal dour & 278238 & eh & 66963 & nice & 190708 & coal & 20448 & dour & 119 \\
\hline eh nice coal dower & 278211 & eh & 66963 & nice & 190708 & coal & 20448 & dower & 92 \\
\hline eh nice cole dour & 259863 & eh & 66963 & nice & 190708 & cole & 2073 & dour & 119 \\
\hline eh nice cole dower & 259836 & eh & 66963 & nice & 190708 & cole & 2073 & dower & 92 \\
\hline eh nice kohl dour & 258702 & eh & 66963 & nice & 190708 & kohl & 912 & dour & 119 \\
\hline eh nice kohl dower & 258675 & eh & 66963 & nice & 190708 & kohl & 912 & dower & 92 \\
\hline ah niece coal dour & 251556 & $\mathrm{ah}$ & 229296 & niece & 1693 & coal & 20448 & dour & 119 \\
\hline ah niece coal dower & 251529 & $\mathrm{ah}$ & 229296 & niece & 1693 & coal & 20448 & dower & 92 \\
\hline ah gneiss coal dour & 249884 & $\mathrm{ah}$ & 229296 & gneiss & 21 & coal & 20448 & dour & 119 \\
\hline ah gneiss coal dower & 249857 & ah & 229296 & gneiss & 21 & coal & 20448 & dower & 92 \\
\hline ah ne skol dour & 236523 & $\mathrm{ah}$ & 229296 & ne & 7103 & skol & 5 & dour & 119 \\
\hline ah ne skol dower & 236496 & $\mathrm{ah}$ & 229296 & ne & 7103 & skol & 5 & dower & 92 \\
\hline ah knee skol dour & 235559 & ah & 229296 & knee & 6139 & skol & 5 & dour & 119 \\
\hline & & & & & & & \multicolumn{3}{|c|}{ Continued on next page } \\
\hline
\end{tabular}


Table B.1 - continued from previous page

\begin{tabular}{|c|c|c|c|c|c|c|c|c|c|}
\hline phrase & total freq & word1 & freq1 & word2 & freq2 & word3 & freq3 & word4 & freq4 \\
\hline ah knee skol dower & 235532 & $\mathrm{ah}$ & 229296 & knee & 6139 & skol & 5 & dower & 92 \\
\hline ah niece cole dour & 233181 & ah & 229296 & niece & 1693 & cole & 2073 & dour & 119 \\
\hline ah niece cole dower & 233154 & ah & 229296 & niece & 1693 & cole & 2073 & dower & 92 \\
\hline ah niece kohl dour & 232020 & ah & 229296 & niece & 1693 & kohl & 912 & dour & 119 \\
\hline ah niece kohl dower & 231993 & ah & 229296 & niece & 1693 & kohl & 912 & dower & 92 \\
\hline ah gneiss cole dour & 231509 & ah & 229296 & gneiss & 21 & cole & 2073 & dour & 119 \\
\hline ah gneiss cole dower & 231482 & ah & 229296 & gneiss & 21 & cole & 2073 & dower & 92 \\
\hline ah nigh skol dour & 230814 & $\mathrm{ah}$ & 229296 & nigh & 1394 & skol & 5 & dour & 119 \\
\hline ah nigh skol dower & 230787 & ah & 229296 & nigh & 1394 & skol & 5 & dower & 92 \\
\hline ah gneiss kohl dour & 230348 & ah & 229296 & gneiss & 21 & kohl & 912 & dour & 119 \\
\hline ah gneiss kohl dower & 230321 & ah & 229296 & gneiss & 21 & kohl & 912 & dower & 92 \\
\hline ah nye skol dour & 229457 & ah & 229296 & nye & 37 & skol & 5 & dour & 119 \\
\hline ah nye skol dower & 229430 & ah & 229296 & nye & 37 & skol & 5 & dower & 92 \\
\hline et nice coal dour & 218006 & et & 6731 & nice & 190708 & coal & 20448 & dour & 119 \\
\hline et nice coal dower & 217979 & et & 6731 & nice & 190708 & coal & 20448 & dower & 92 \\
\hline o' nice coal dour & 211738 & $\mathrm{o}^{\prime}$ & 463 & nice & 190708 & coal & 20448 & dour & 119 \\
\hline o' nice coal dower & 211711 & o' & 463 & nice & 190708 & coal & 20448 & dower & 92 \\
\hline 'n' aye scold hour & 202533 & 'n' & 0 & aye & 130503 & scold & 217 & hour & 71813 \\
\hline et nice cole dour & 199631 & et & 6731 & nice & 190708 & cole & 2073 & dour & 119 \\
\hline et nice cole dower & 199604 & et & 6731 & nice & 190708 & cole & 2073 & dower & 92 \\
\hline et nice kohl dour & 198470 & et & 6731 & nice & 190708 & kohl & 912 & dour & 119 \\
\hline et nice kohl dower & 198443 & et & 6731 & nice & 190708 & kohl & 912 & dower & 92 \\
\hline
\end{tabular}


Table B.1 - continued from previous page

\begin{tabular}{cc|cccccccc}
\hline phrase & total freq & word1 & freq1 & word2 & freq2 & word3 & freq3 & word4 & freq4 \\
\hline o' nice cole dour & 193363 & o' & 463 & nice & 190708 & cole & 2073 & dour & 119 \\
\hline o' nice cole dower & 193336 & o' & 463 & nice & 190708 & cole & 2073 & dower & 92 \\
\hline eh niece cold hour & 193313 & eh & 66963 & niece & 1693 & cold & 52844 & hour & 71813 \\
\hline o' nice kohl dour & 192202 & o' & 463 & nice & 190708 & kohl & 912 & dour & 119 \\
\hline o' nice kohl dower & 192175 & o' & 463 & nice & 190708 & kohl & 912 & dower & 92 \\
\hline eh gneiss cold hour & 191641 & eh & 66963 & gneiss & 21 & cold & 52844 & hour & 71813 \\
\hline 'n' e scold hour & 180993 & 'n' & 0 & e & 108963 & scold & 217 & hour & 71813 \\
\hline eh ne scold hour & 146096 & eh & 66963 & ne & 7103 & scold & 217 & hour & 71813 \\
\hline eh knee scold hour & 145132 & eh & 66963 & knee & 6139 & scold & 217 & hour & 71813 \\
\hline eh nigh scold hour & 140387 & eh & 66963 & nigh & 1394 & scold & 217 & hour & 71813 \\
\hline eh nye scold hour & 139030 & eh & 66963 & nye & 37 & scold & 217 & hour & 71813 \\
\hline 'n' ice cold hour & 136859 & 'n' & 0 & ice & 12202 & cold & 52844 & hour & 71813 \\
\hline et niece cold hour & 133081 & et & 6731 & niece & 1693 & cold & 52844 & hour & 71813 \\
\hline et gneiss cold hour & 131409 & et & 6731 & gneiss & 21 & cold & 52844 & hour & 71813 \\
\hline 'n' aye skol dour & 130627 & 'n' & 0 & aye & 130503 & skol & 5 & dour & 119 \\
\hline 'n' aye skol dower & 130600 & 'n' & 0 & aye & 130503 & skol & 5 & dower & 92 \\
\hline o' niece cold hour & 126813 & o' & 463 & niece & 1693 & cold & 52844 & hour & 71813 \\
\hline o' gneiss cold hour & 125141 & o' & 463 & gneiss & 21 & cold & 52844 & hour & 71813 \\
\hline 'n' e skol dour & 109087 & 'n' & 0 & e & 108963 & skol & 5 & dour & 119 \\
\hline 'n' e skol dower & 109060 & 'n' & 0 & e & 108963 & skol & 5 & dower & 92 \\
\hline eh' eye scold hour & 98780 & 'n' & 0 & eye & 26750 & scold & 217 & hour & 71813 \\
\hline & & & & & & & Continued on next page \\
\hline
\end{tabular}


Table B.1 - continued from previous page

\begin{tabular}{|c|c|c|c|c|c|c|c|c|c|}
\hline phrase & total freq & word1 & freq1 & word2 & freq2 & word3 & freq3 & word4 & freq4 \\
\hline eh niece coal dower & 89196 & eh & 66963 & niece & 1693 & coal & 20448 & dower & 92 \\
\hline eh gneiss coal dour & 87551 & eh & 66963 & gneiss & 21 & coal & 20448 & dour & 119 \\
\hline eh gneiss coal dower & 87524 & eh & 66963 & gneiss & 21 & coal & 20448 & dower & 92 \\
\hline et ne scold hour & 85864 & et & 6731 & ne & 7103 & scold & 217 & hour & 71813 \\
\hline et knee scold hour & 84900 & et & 6731 & knee & 6139 & scold & 217 & hour & 71813 \\
\hline et nigh scold hour & 80155 & et & 6731 & nigh & 1394 & scold & 217 & hour & 71813 \\
\hline o' ne scold hour & 79596 & $\mathrm{o}^{\prime}$ & 463 & ne & 7103 & scold & 217 & hour & 71813 \\
\hline et nye scold hour & 78798 & et & 6731 & nye & 37 & scold & 217 & hour & 71813 \\
\hline 'n' ay scold hour & 78663 & 'n' & 0 & ay & 6633 & scold & 217 & hour & 71813 \\
\hline o' knee scold hour & 78632 & o' & 463 & knee & 6139 & scold & 217 & hour & 71813 \\
\hline eh ne skol dour & 74190 & eh & 66963 & ne & 7103 & skol & 5 & dour & 119 \\
\hline eh ne skol dower & 74163 & eh & 66963 & ne & 7103 & skol & 5 & dower & 92 \\
\hline o' nigh scold hour & 73887 & $\mathrm{o}^{\prime}$ & 463 & nigh & 1394 & scold & 217 & hour & 71813 \\
\hline eh knee skol dour & 73226 & eh & 66963 & knee & 6139 & skol & 5 & dour & 119 \\
\hline eh knee skol dower & 73199 & eh & 66963 & knee & 6139 & skol & 5 & dower & 92 \\
\hline o' nye scold hour & 72530 & $\mathrm{o}^{\prime}$ & 463 & nye & 37 & scold & 217 & hour & 71813 \\
\hline 'n' ice-cold hour & 71862 & 'n' & 0 & ice-cold & 49 & hour & 71813 & & \\
\hline eh niece cole dour & 70848 & eh & 66963 & niece & 1693 & cole & 2073 & dour & 119 \\
\hline eh niece cole dower & 70821 & eh & 66963 & niece & 1693 & cole & 2073 & dower & 92 \\
\hline eh niece kohl dour & 69687 & eh & 66963 & niece & 1693 & kohl & 912 & dour & 119 \\
\hline eh niece kohl dower & 69660 & eh & 66963 & niece & 1693 & kohl & 912 & dower & 92 \\
\hline eh gneiss cole dour & 69176 & eh & 66963 & gneiss & 21 & cole & 2073 & dour & 119 \\
\hline & & & & & & & \multicolumn{3}{|c|}{ Continued on next page } \\
\hline
\end{tabular}


Table B.1 - continued from previous page

\begin{tabular}{|c|c|c|c|c|c|c|c|c|c|}
\hline phrase & total freq & word1 & freq1 & word2 & freq2 & word3 & freq3 & word4 & freq4 \\
\hline eh gneiss cole dower & 69149 & eh & 66963 & gneiss & 21 & cole & 2073 & dower & 92 \\
\hline eh nigh skol dour & 68481 & eh & 66963 & nigh & 1394 & skol & 5 & dour & 119 \\
\hline eh nigh skol dower & 68454 & eh & 66963 & nigh & 1394 & skol & 5 & dower & 92 \\
\hline eh gneiss kohl dour & 68015 & eh & 66963 & gneiss & 21 & kohl & 912 & dour & 119 \\
\hline eh gneiss kohl dower & 67988 & eh & 66963 & gneiss & 21 & kohl & 912 & dower & 92 \\
\hline eh nye skol dour & 67124 & eh & 66963 & nye & 37 & skol & 5 & dour & 119 \\
\hline eh nye skol dower & 67097 & eh & 66963 & nye & 37 & skol & 5 & dower & 92 \\
\hline 'n' ice coal dour & 32769 & 'n' & 0 & ice & 12202 & coal & 20448 & dour & 119 \\
\hline 'n' ice coal dower & 32742 & 'n' & 0 & ice & 12202 & coal & 20448 & dower & 92 \\
\hline et niece coal dour & 28991 & et & 6731 & niece & 1693 & coal & 20448 & dour & 119 \\
\hline et niece coal dower & 28964 & et & 6731 & niece & 1693 & coal & 20448 & dower & 92 \\
\hline et gneiss coal dour & 27319 & et & 6731 & gneiss & 21 & coal & 20448 & dour & 119 \\
\hline et gneiss coal dower & 27292 & et & 6731 & gneiss & 21 & coal & 20448 & dower & 92 \\
\hline 'n' eye skol dour & 26874 & 'n' & 0 & eye & 26750 & skol & 5 & dour & 119 \\
\hline 'n' eye skol dower & 26847 & 'n' & 0 & eye & 26750 & skol & 5 & dower & 92 \\
\hline o' niece coal dour & 22723 & o' & 463 & niece & 1693 & coal & 20448 & dour & 119 \\
\hline o' niece coal dower & 22696 & $\mathrm{o}^{\prime}$ & 463 & niece & 1693 & coal & 20448 & dower & 92 \\
\hline o' gneiss coal dour & 21051 & $\mathrm{o}^{\prime}$ & 463 & gneiss & 21 & coal & 20448 & dour & 119 \\
\hline o' gneiss coal dower & 21024 & o' & 463 & gneiss & 21 & coal & 20448 & dower & 92 \\
\hline 'n' ice cole dour & 14394 & 'n' & 0 & ice & 12202 & cole & 2073 & dour & 119 \\
\hline 'n' ice cole dower & 14367 & 'n' & 0 & ice & 12202 & cole & 2073 & dower & 92 \\
\hline et ne skol dour & 13958 & et & 6731 & ne & 7103 & skol & 5 & dour & 119 \\
\hline & & & & & & & \multicolumn{3}{|c|}{ Continued on next page } \\
\hline
\end{tabular}


Table B.1 - continued from previous page

\begin{tabular}{cc|cccccccc}
\hline phrase & total freq & word1 & freq1 & word2 & freq2 & word3 & freq3 & word4 & freq4 \\
\hline et ne skol dower & 13931 & et & 6731 & ne & 7103 & skol & 5 & dower & 92 \\
\hline 'n' ice kohl dour & 13233 & 'n' & 0 & ice & 12202 & kohl & 912 & dour & 119 \\
\hline 'n' ice kohl dower & 13206 & 'n' & 0 & ice & 12202 & kohl & 912 & dower & 92 \\
\hline et knee skol dour & 12994 & et & 6731 & knee & 6139 & skol & 5 & dour & 119 \\
\hline et knee skol dower & 12967 & et & 6731 & knee & 6139 & skol & 5 & dower & 92 \\
\hline et niece cole dour & 10616 & et & 6731 & niece & 1693 & cole & 2073 & dour & 119 \\
\hline et niece cole dower & 10589 & et & 6731 & niece & 1693 & cole & 2073 & dower & 92 \\
\hline et niece kohl dour & 9455 & et & 6731 & niece & 1693 & kohl & 912 & dour & 119 \\
\hline et niece kohl dower & 9428 & et & 6731 & niece & 1693 & kohl & 912 & dower & 92 \\
\hline et gneiss cole dour & 8944 & et & 6731 & gneiss & 21 & cole & 2073 & dour & 119 \\
\hline et gneiss cole dower & 8917 & et & 6731 & gneiss & 21 & cole & 2073 & dower & 92 \\
\hline et nigh skol dour & 8249 & et & 6731 & nigh & 1394 & skol & 5 & dour & 119 \\
\hline et nigh skol dower & 8222 & et & 6731 & nigh & 1394 & skol & 5 & dower & 92 \\
\hline et gneiss kohl dour & 7783 & et & 6731 & gneiss & 21 & kohl & 912 & dour & 119 \\
\hline et gneiss kohl dower & 7756 & et & 6731 & gneiss & 21 & kohl & 912 & dower & 92 \\
\hline o' ne skol dour & 7690 & o' & 463 & ne & 7103 & skol & 5 & dour & 119 \\
\hline o' ne skol dower & 7663 & o' & 463 & ne & 7103 & skol & 5 & dower & 92 \\
\hline et nye skol dour & 6892 & et & 6731 & nye & 37 & skol & 5 & dour & 119 \\
\hline et nye skol dower & 6865 & et & 6731 & nye & 37 & skol & 5 & dower & 92 \\
\hline 'n' ay skol dour & 6757 & 'n' & 0 & ay & 6633 & skol & 5 & dour & 119 \\
\hline 'n' ay skol dower & 6730 & 'n' & 0 & ay & 6633 & skol & 5 & dower & 92 \\
\hline o' knee skol dour & 6726 & o' & 463 & knee & 6139 & skol & 5 & dour & 119 \\
\hline & & & & & & Continued on next page \\
\hline
\end{tabular}


Table B.1 - continued from previous page

\begin{tabular}{cc|cccccccc}
\hline phrase & total freq & word1 & freq1 & word2 & freq2 & word3 & freq3 & word4 & freq4 \\
\hline o' knee skol dower & 6699 & o' & 463 & knee & 6139 & skol & 5 & dower & 92 \\
\hline o' niece cole dour & 4348 & o' & 463 & niece & 1693 & cole & 2073 & dour & 119 \\
\hline o' niece cole dower & 4321 & o' & 463 & niece & 1693 & cole & 2073 & dower & 92 \\
\hline o' niece kohl dour & 3187 & o' & 463 & niece & 1693 & kohl & 912 & dour & 119 \\
\hline o' niece kohl dower & 3160 & o' & 463 & niece & 1693 & kohl & 912 & dower & 92 \\
\hline o' gneiss cole dour & 2676 & o' & 463 & gneiss & 21 & cole & 2073 & dour & 119 \\
\hline o' gneiss cole dower & 2649 & o' & 463 & gneiss & 21 & cole & 2073 & dower & 92 \\
\hline o' nigh skol dour & 1981 & o' & 463 & nigh & 1394 & skol & 5 & dour & 119 \\
\hline o' nigh skol dower & 1954 & o' & 463 & nigh & 1394 & skol & 5 & dower & 92 \\
\hline o' gneiss kohl dour & 1515 & o' & 463 & gneiss & 21 & kohl & 912 & dour & 119 \\
\hline o' gneiss kohl dower & 1488 & o' & 463 & gneiss & 21 & kohl & 912 & dower & 92 \\
\hline o' nye skol dour & 624 & o' & 463 & nye & 37 & skol & 5 & dour & 119 \\
\hline o' nye skol dower & 597 & o' & 463 & nye & 37 & skol & 5 & dower & 92 \\
\hline
\end{tabular}

Table B.1: All Oronyms for 'A Nice Cold Hour' with frequency values 


\section{Appendix C}

\section{SAMPA Phonetic Alphabet}

Table C.1: Full Table of SAMPA Phoneme Makeup and Length, with examples for each phoneme. 


\section{Full Table of SAMPA Phoneme Makeup and Length}

\begin{tabular}{|c|c|c|c|c|c|c|c|}
\hline SAMPA & Example & $\begin{array}{c}\text { Type/ } \\
\text { Manner of } \\
\text { Articulation }\end{array}$ & Voiced/ & & & & Weight \\
\hline p & pen, spin, tip & plosive & voiceless & block & $\begin{array}{l}\text { Enas as } \\
\text { block }\end{array}$ & Con. & 8 \\
\hline b & but, web & plosive & voiced & block & block & Con. & 7 \\
\hline$t$ & two, sting, bet & plosive & voiceless & block & block & Con. & 8 \\
\hline d & do, odd & plosive & voiced & block & block & Con. & 7 \\
\hline ts & chair, nature, teach & affricate & voiceless & block & cont fric & Con. & 6 \\
\hline $\mathrm{dz}$ & gin, joy, edge & affricate & voiced & block & cont fric & Con. & 5 \\
\hline $\mathbf{k}$ & cat, kill, queen, thick & plosive & voiceless & block & block & Con. & 8 \\
\hline g & go, get, beg & plosive & voiced & block & block & Con. & 7 \\
\hline$f$ & fool, enough, leaf & fricative & voiceless & cont fric & cont fric & Con. & 4 \\
\hline $\mathbf{v}$ & voice, have, of & fricative & voiced & cont fric & cont fric & Con. & 3 \\
\hline $\mathbf{T}$ & thing, breath & fricative & voiceless & cont fric & cont fric & Con. & 4 \\
\hline D & this, breathe & fricative & voiced & cont fric & cont fric & Con. & 3 \\
\hline $\mathbf{s}$ & see, city, pass & fricative & voiceless & cont fric & cont fric & Con. & 4 \\
\hline $\mathbf{z}$ & zoo, rose & fricative & voiced & cont fric & cont fric & Con. & 3 \\
\hline $\mathbf{s}$ & she, sure, emotion, leash & fricative & voiceless & cont fric & cont fric & Con. & 4 \\
\hline $\mathbf{z}$ & pleasure, beige & fricative & voiced & cont fric & cont fric & Con. & 3 \\
\hline $\mathbf{h}$ & ham & fricative & voiceless & cont fric & cont fric & Con. & 4 \\
\hline m & man, ham & nasal & voiced & redirect & redirect & Con. & 1 \\
\hline $\mathbf{n}$ & no, tin & nasal & voiced & redirect & redirect & Con. & 1 \\
\hline $\mathbf{N}$ & singer, ring & nasal & voiced & redirect & redirect & Con. & 1 \\
\hline 1 & left, bell & lateral & voiced & continuous & continuous & Con. & 0 \\
\hline$r$ & run, very & approximant & voiced & continuous & continuous & Con. & 0 \\
\hline w & we & semivowel & voiced & continuous & end & Con. & 2 \\
\hline j & yes & semivowel & voiced & continuous & end & Con. & 2 \\
\hline $\mathrm{W}$ & what (Scottish) & approximant & voiceless & continuous & end & Con. & 3 \\
\hline $\mathbf{x}$ & loch (Scottish) & fricative & voiceless & cont fric & cont fric & Con. & 4 \\
\hline $\mathbf{A}$ & father, not, law & short & & & & Vow. & 0.25 \\
\hline I & city & short & & & & Vow. & 0.25 \\
\hline $\mathbf{E}$ & bed & short & & & & Vow. & 0.25 \\
\hline $3 \% r$ & bird, winner & short & & & & Vow. & 0.25 \\
\hline\{ & lad, cat, ran & short & & & & Vow. & 0.25 \\
\hline $\mathbf{u}$ & soon, through & short & & & & Vow. & 0.25 \\
\hline a & about & short & & & & Vow. & 0.25 \\
\hline$j u / j u$ & use, pupil & diphthong & & semivowel & short & Dip. & 0.5 \\
\hline$i$ & see & long & & & & Vow. & 0.75 \\
\hline $\mathbf{v}$ & run, enough & short & & & & Vow. & 0.25 \\
\hline $\mathbf{U}$ & put & long & & & & Vow. & 0.75 \\
\hline e & day & long & & & & Vow. & 0.75 \\
\hline 0 & or, shore & long & & & & Vow. & 0.75 \\
\hline $\mathbf{a}$ & DNE in GenAm & long & & & & Vow. & 0.75 \\
\hline aI & my, height & diphthong & & long & short & Dip. & 1 \\
\hline OI & boy & diphthong & & long & short & Dip. & 1 \\
\hline ou/ou & boat & diphthong & & short & long & Dip. & 1 \\
\hline $\mathrm{aU}$ & now & diphthong & & long & long & Dip. & 1.5 \\
\hline$=$ & ridden & semivowel & & & & Vow. & 0.25 \\
\hline
\end{tabular}




\section{Appendix D}

\section{User Study Details}

\begin{tabular}{|c|c|c|}
\hline orthoPhrase & numRecordings & phraseID \\
\hline a nice cold our & 3 & A.17.51 a nice cold our \\
\hline an ice cold our & 2 & A.17.135 an ice cold our \\
\hline a nye scold our & 2 & A.17.69 a nye scold our \\
\hline ah nye scold our & 2 & A.18.109 ah nye scold our \\
\hline an eye scold our & 2 & A.18.125 an eye scold our \\
\hline on aye scold our & 2 & A.18.267 on aye scold our \\
\hline a nigh scold our & 2 & A.18.65 a nigh scold our \\
\hline a nye skol dower & 2 & A.18.71 a nye skol dower \\
\hline an aye skol dower & 2 & A.19.119 an aye skol dower \\
\hline an eye skol dower & 2 & A.19.127 an eye skol dower \\
\hline an ice coal dower & 2 & A.19.133 an ice coal dower \\
\hline eh nice coal dower & 2 & A.20.159 eh nice coal dower \\
\hline ah nice coal dower & 2 & A.20.89 ah nice coal dower \\
\hline & & Continued on next page \\
\hline
\end{tabular}


Table D.1 - continued from previous page

\begin{tabular}{|c|c|c|}
\hline orthoPhrase & numRecordings & phraseID \\
\hline fourth wry to & 2 & B.15.19 fourth wry to \\
\hline fourth wry too & 2 & B.16.20 fourth wry too \\
\hline forth right ooh & 2 & B.17.1 forth right ooh \\
\hline fourth rite ooh & 2 & B.17.13 fourth rite ooh \\
\hline forth wright ooh & 2 & B.18.6 forth wright ooh \\
\hline on i scold our & 1 & A.16.279 on i scold our \\
\hline an i scold hour & 1 & A.17.128 an i scold hour \\
\hline an i skol dower & 1 & A.17.131 an i skol dower \\
\hline an ice-cold our & 1 & A.17.141 an ice-cold our \\
\hline on i scold hour & 1 & A.17.278 on i scold hour \\
\hline on i skol dower & 1 & A.17.281 on i skol dower \\
\hline an aye scold our & 1 & A.18.117 an aye scold our \\
\hline an ice cold hour & 1 & A.18.134 an ice cold hour \\
\hline an ice-cold hour & 1 & A.18.140 an ice-cold hour \\
\hline eh nye scold our & 1 & A.18.179 eh nye scold our \\
\hline on eye scold our & 1 & A.18.275 on eye scold our \\
\hline on ice cold hour & 1 & A.18.284 on ice cold hour \\
\hline on ice-cold hour & 1 & A.18.290 on ice-cold hour \\
\hline a nye scold hour & 1 & A.18.68 a nye scold hour \\
\hline ah nice cold our & 1 & A.18.91 ah nice cold our \\
\hline ah nigh scold our & 1 & A.19.105 ah nigh scold our \\
\hline ah nye scold hour & 1 & A.19.108 ah nye scold hour \\
\hline & & Continued on next page \\
\hline
\end{tabular}


Table D.1 - continued from previous page

\begin{tabular}{|c|c|c|}
\hline orthoPhrase & numRecordings & phraseID \\
\hline ah nye skol dower & 1 & A.19.111 ah nye skol dower \\
\hline an aye scold hour & 1 & A.19.116 an aye scold hour \\
\hline an ice kohl dower & 1 & A.19.139 an ice kohl dower \\
\hline eh nice cold hour & 1 & A.19.160 eh nice cold hour \\
\hline eh nigh scold our & 1 & A.19.175 eh nigh scold our \\
\hline eh nye skol dower & 1 & A.19.181 eh nye skol dower \\
\hline on aye skol dower & 1 & A.19.269 on aye skol dower \\
\hline on eye scold hour & 1 & A.19.274 on eye scold hour \\
\hline on ice coal dower & 1 & A.19.283 on ice coal dower \\
\hline on ice kohl dower & 1 & A.19.289 on ice kohl dower \\
\hline a nice coal dower & 1 & A.19.49 a nice coal dower \\
\hline a nigh scold hour & 1 & A.19.64 a nigh scold hour \\
\hline ah nice cold hour & 1 & A.19.90 ah nice cold hour \\
\hline eh nice cole dower & 1 & A.20.163 eh nice cole dower \\
\hline eh nigh scold hour & 1 & A.20.174 eh nigh scold hour \\
\hline eh nigh skol dower & 1 & A.20.177 eh nigh skol dower \\
\hline ah nice cole dower & 1 & A.20.93 ah nice cole dower \\
\hline ah nice kohl dower & 1 & A.20.95 ah nice kohl dower \\
\hline forth wry two & 1 & B.15.10 forth wry two \\
\hline forth rye two & 1 & B.15.5 forth rye two \\
\hline forth write ooh & 1 & B.17.7 forth write ooh \\
\hline fourth right ooh & 1 & B.18.12 fourth right ooh \\
\hline & & Continued on next page \\
\hline
\end{tabular}


Table D.1 - continued from previous page

\begin{tabular}{|c|c|c|}
\hline orthoPhrase & numRecordings & phraseID \\
\hline fourth wright ooh & 1 & B.19.17 fourth wright ooh \\
\hline
\end{tabular}

Table D.1: Here are the phrases we recorded, how many times they were recorded, and the identifiers we used for each phrase 


\begin{tabular}{|c|c|}
\hline Response By Country & Num Responses \\
\hline USA & 506 \\
\hline India & 277 \\
\hline Canada & 33 \\
\hline England & 28 \\
\hline Macedonia & 28 \\
\hline Washington & 13 \\
\hline Syria & 13 \\
\hline UK & 11 \\
\hline Sri Lanka & 7 \\
\hline Britain & 4 \\
\hline Vietnam & 4 \\
\hline Egypt & 3 \\
\hline Finland & 3 \\
\hline Iran & 3 \\
\hline Phillipines & 3 \\
\hline The Netherlands & 2 \\
\hline Mexico & 2 \\
\hline "english" & 2 \\
\hline Belgium & 2 \\
\hline Ireland & 2 \\
\hline Other & 8 \\
\hline
\end{tabular}

Table D.2: Here's a table with the number of responses per country 


\section{Bibliography}

[1] All our n-gram are belong to you | research blog. http://googleresearch.blogspot.com/2006/08/all-our-n-gram-are-belongto-you.html.

[2] The CMU pronouncing dictionary. http://www.speech.cs.cmu.edu/cgibin/cmudict.

[3] Corpus-based word frequency lists, collocates, and n-grams. http://www.wordfrequency.info/comparison.asp.

[4] Dear R/Assistance, i'm about to finish my master's thesis, but i need your help! (tasks are online; i'm in san luis obispo, CA). : Assistance. http://www.reddit.com/r/Assistance/comments/ubty1/dear_rassistance_im_about_to_finish_m

[5] Dear RecordThis: i'm finishing up my masters thesis, and i need your help! : recordthis. http://www.reddit.com/r/recordthis/comments/ubt9f/dear_recordthis_im_finishing_up_my_ma

[6] File:General american.png - wikipedia, the free encyclopedia. http://en.wikipedia.org/wiki/File:General_American.png.

[7] Keep thou my way, hymnlyrics.org. http://www.hymnlyrics.org/newlyrics_k/keep_thou_my_way [8] knights_emic.gif (GIF image, 552407 pixels) - scaled (0\%). 
[9] knights_phonetic.jpg (JPEG image, 552407 pixels) - scaled (0\%).

[10] LCStar project web - schedule. http://www.lc-star.com/schedule.htm.

[11] Mondegreen | define mondegreen at dictionary.com. http://dictionary.reference.com/browse/mondegreen?s=t.

[12] N-grams: corpus based (COCA, COHA, spanish, portuguese). http://www.ngrams.info/.

[13] oronym - definition and meaning. http://www.wordnik.com/words/oronym.

[14] Orthography | define orthography at dictionary.com. http://dictionary.reference.com/browse/orthography.

[15] SQLite database browser. http://sqlitebrowser.sourceforge.net/.

[16] Understanding how to sing the vowels - a technical description for classical singers. http://ezinearticles.com/?Understanding-How-to-Sing-theVowels-A-Technical-Description-For-Classical-Singers\&id=1671860.

[17] Unisyn lexicon. http://www.cstr.ed.ac.uk/projects/unisyn/.

[18] Why standard american english really is "no accent". http://boards.straightdope.com/sdmb/archive/index.php/t-619668.html.

[19] 'At the tone' it will be jane barbe, america's answer to big ben. People Magazine, Aug. 1976.

[20] (1) "Rolling in the deep" cover, front porch band, Jan. 2012.

[21] J. Aronson. When i use a word words misheard: Medical mondegreens. QJM, 102(4):301-302, Apr. 2009. 
[22] D. Crystal. DCblog: on singing accents, Nov. 2009.

[23] M. Davies. Word frequency data from the corpus of contemporary american english (COCA)., 2011.

[24] G. W. Elko, J. Meyer, S. Backer, and J. Peissig. Electronic pop protection for microphones. In Applications of Signal Processing to Audio and Acoustics, 2007 IEEE Workshop on, pages 46 -49, Oct. 2007.

[25] S. Fitt. Documentation and user guide to UNISYN lexicon and post-lexical rules. Center for Speech Technology Research, University of Edinburgh, Tech. Rep, 2000.

[26] J. Hendrix. Purple haze, June 1967.

[27] H. Lieberman, A. Faaborg, W. Daher, and J. Espinosa. How to wreck a nice beach you sing calm incense. In Proceedings of the 10th international conference on Intelligent user interfaces, page 278280, 2005.

[28] C. C. Revival. Bad moon rising, Apr. 1969.

[29] G. P. Smith. Music and mondegreens: extracting meaning from noise. ELT Journal, 57(2):113121, 2003.

[30] J. Sprouse. A validation of amazon mechanical turk for the collection of acceptability judgments in linguistic theory. Behavior Research Methods, 43(1):155-167, 2011.

[31] S. Wright. The death of lady mondegreen. Harpers Magazine, 209(1254):4851, 1954. 